\title{
NRC's Object-Oriented
}

Simulator Instructor Station

\section{U.S. Nuclear Regulatory Commission}

Office for Analysis and Evaluation of Operational Data

\author{
J. I. Griffin, J. P. Griffin
}

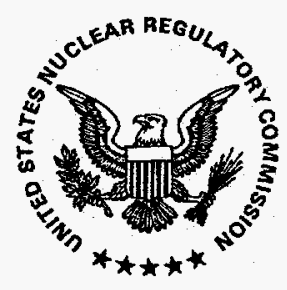




\section{AVAILABILITY NOTICE}

Availability of Reference Materials Cited in NRC Publications

Most documents cited in NRC publications will be available from one of the following sources:

1. The NRC Public Document Room, 2120 L Street, NW., Lower Level, Washington, DC 20555-0001

2. The Superintendent of Documents, U.S. Government Printing Office, P. O. Box 37082 , Washington, DC 20402-9328

3. The National Technical information Service, Springfield, VA 22161-0002

Although the listing that follows represents the majority of documents cited in NRC publications, it is not intended to be exhaustive.

Referenced documents available for inspection and copying for a fee from the NRC Public Document Room include NRC correspondence and internal NRC memoranda; NRC bulletins, circulars, information notices, inspection and investigation notices; licensee event reports; vendor reports and correspondence; Commission papers; and applicant and licensee documents and correspondence.

The following documents in the NUREG series are available for purchase from the Government Printing Office: formal NRC staff and contractor reports, NRC-sponsored conference proceedings, international agreement reports, grantee reports, and NRC booklets and brochures. Also available are regulatory guides, NRC regulations in the Code of Federal Regulations, and Nuclear Regulatory Commission issuances.

Docurnents available from the National Technical Information Service include NUREG-series reports and technical reports prepared by other Federal agencies and reports prepared by the Atomic Energy Commission, forerunner agency to the Nuclear Regulatory Commission.

Documents available from public and special technical libraries include all open literature items, such as books, journal articles, and transactions. Federal Register notices, Federal and State legislation, and congressional reports can usually be obtained from these libraries.

Docurnents such as theses, dissertations, foreign reports and translations, and non-NRC conference proceedings are available for purchase from the organization sponsoring the publication cited.

Single copies of NRC draft reports are available free, to the extent of supply, upon written request to the Office of Administration, Distribution and Mail Services Section, U.S. Nuclear Regulatory Commission, Washington DC 20555-0001.

Copies of industry codes and standards used in a substantive manner in the NRC regulatory process are maintained at the NRC Library, Two White Flint North, 11545 Rockville Pike, Rockville, MD 20852-2738, for use by the public. Codes and standards are usually copyrighted and may be purchased from the originating organization or, if they are American National Standards, from the American National Standards Institute, 1430 Broadway, New York, NY 10018-3308. 


\section{NRC's Object-Oriented Simulator Instructor Station}

Manuscript Completed: May 1995

Date Published: June 1995

\section{J. I. Griffin, J. P. Griffin}

Technical Training Division

Office for Analysis and Evaluation of Operational Data

U.S. Nuclear Regulatory Commission

Washington, DC 20555-0001

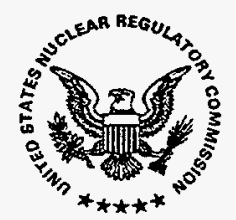





\section{DISCLAIMER}

This report was prepared as an account of work sponsored by an agency of the United States Government. Neither the United States Government nor any agency thereof, nor any of their employees, make any warranty, express or implied, or assumes any legal liability or responsibility for the accuracy, completeness, or usefulness of any information, apparatus, product, or process disclosed, or represents that its use would not infringe privately owned rights. Reference herein to any specific commercial product, process, or service by trade name, trademark, manufacturer, or otherwise does not necessarily constitute or imply its endorsement, recommendation, or favoring by the United States Government or any agency thereof. The views and opinions of authors expressed herein do not necessarily state or reflect those of the United States Government or any agency thereof. 


\section{DISCLAIMER}

Portions of this document may be illegible in electronic image products. Images are produced from the best available original document. 


\begin{abstract}
As part of a comprehensive simulator upgrade program, the simulator computer systems associated with the Nuclear Regulatory Commission's (NRC) nuclear power plant simulators were replaced. Because the original instructor stations for two of the simulators were dependent on the original computer equipment, it was necessary to develop and implement new instructor stations. This report describes the Macintosh-based Instructor Stations developed by NRC engineers for the General Electric (GE) and Babcock and Wilcox (B\&W) simulators.
\end{abstract}





\section{TABLE OF CONTENTS}

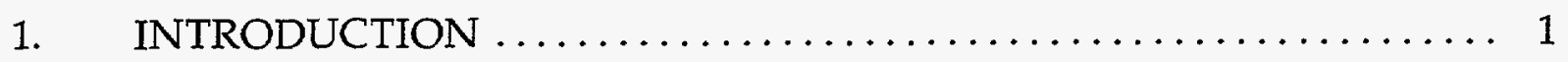

1.1 Instructor Station Upgrade Background $\ldots \ldots \ldots \ldots \ldots \ldots \ldots, 1$

1.2 Implementation Choices ........................ 2

2. MACINTOSH INSTRUCTOR STATION SOFTWARE $\ldots \ldots \ldots \ldots \ldots$.

$2.1 \quad$ Host Computer Software ........................ 4

2.1.1 MACPCM and CMDH $\ldots \ldots \ldots \ldots \ldots \ldots \ldots \ldots \ldots \ldots, 4$

2.1 .2 MALFHAND ............................ 7

2.1.3 Support Programs/Subroutines $\ldots \ldots \ldots \ldots \ldots \ldots \ldots \ldots$

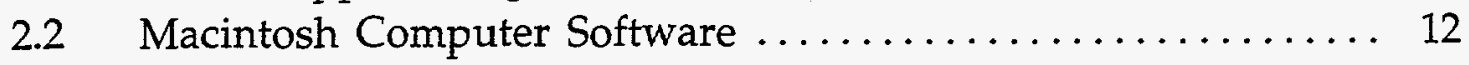

2.2.1 SuperCard Concepts ........................... 12

2.2.2 SuperCard Implementation $\ldots \ldots \ldots \ldots \ldots \ldots \ldots \ldots \ldots, 14$

2.3 Communication Protocol ....................... 15

Table 2-1 MACPCM Commands $\ldots \ldots \ldots \ldots \ldots \ldots \ldots \ldots \ldots$

3. MACINTOSH INSTRUCTOR STATION CAPABILITIES $\ldots \ldots \ldots \ldots$

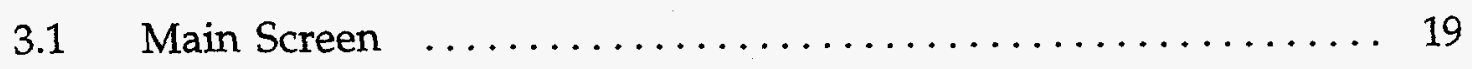

3.1.1 Simulator Status Summary $\ldots \ldots \ldots \ldots \ldots \ldots \ldots \ldots \ldots$

3.1.2 Control Palette ......................... 19

3.1.3 Menu Bar ............................ 20

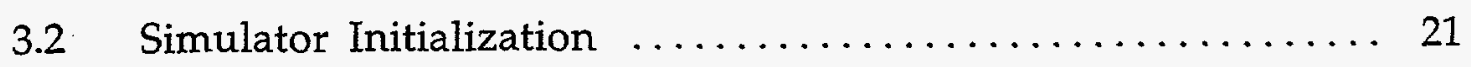

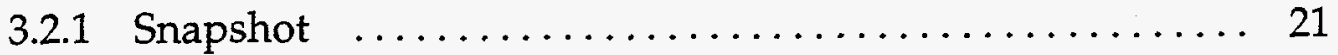

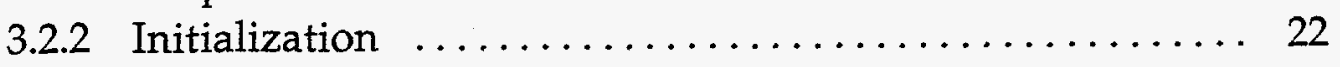

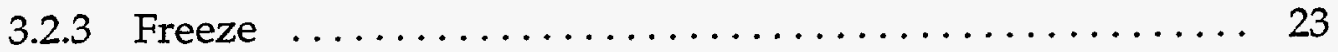

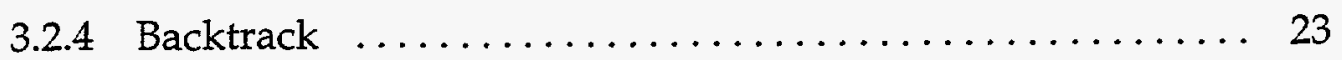

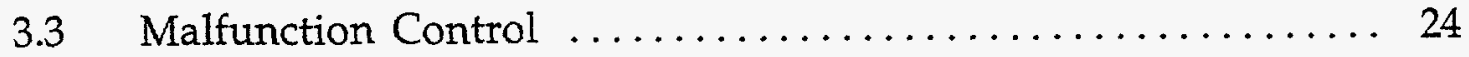

3.3.1 Available Malfunctions $\ldots \ldots \ldots \ldots \ldots \ldots \ldots \ldots \ldots .25$

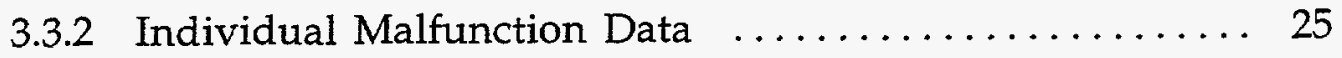

3.3.3 List of Activated Malfunctions ................. 26

3.3.4 Malfunction Activation and Change $\ldots \ldots \ldots \ldots \ldots .27$

3.3.5 Malfunction Deletion ...................... 28

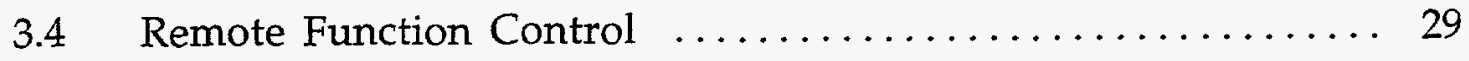

3.4.1 Available Remote Functions ................ 29 
3.4.2 Individual Remote Function Data $\ldots \ldots \ldots \ldots \ldots \ldots .30$

3.4.3 Remote Function Operation $\ldots \ldots \ldots \ldots \ldots \ldots \ldots \ldots, 30$

$3.5 \quad$ I/O Override Control $\ldots \ldots \ldots \ldots \ldots \ldots \ldots \ldots \ldots \ldots . . \ldots \ldots$

3.5.1 Available I/O Overrides .................... 32

3.5.2 Individual I/O Override Data $\ldots \ldots \ldots \ldots \ldots \ldots \ldots, 32$

3.5.3 List of Active Overrides .................... 33

3.5 .4 I/O Override Activation $\ldots \ldots \ldots \ldots \ldots \ldots \ldots \ldots . \ldots \ldots$

3.5 .5 I/O Override Deletion $\ldots \ldots \ldots \ldots \ldots \ldots \ldots \ldots \ldots \ldots . \ldots \ldots$

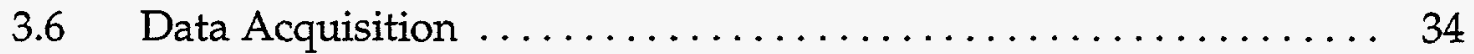

3.6.1 Data Recording .......................... 34

3.6.2 Data Conversion and Transfer to Excel ........... 36

3.6.3 Data File Manipulation Using Excel ............. 37

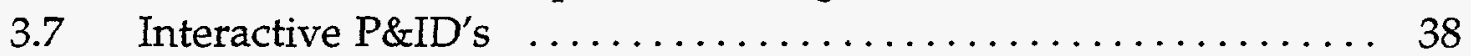

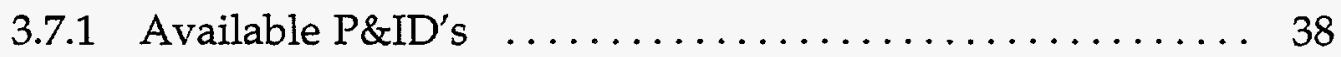

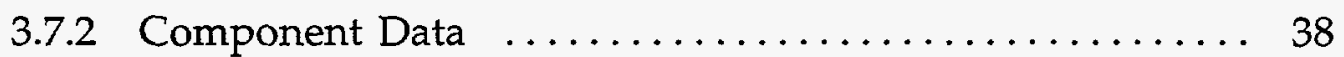

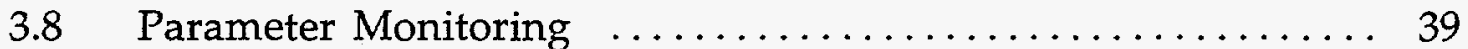

3.8.1 Datapool Variable Selection $\ldots \ldots \ldots \ldots \ldots \ldots \ldots . . \ldots \ldots$

3.8.2 Start of Parameter Monitoring $\ldots \ldots \ldots \ldots \ldots \ldots \ldots .40$

3.8.3 Addition or Deletion of Variables $\ldots \ldots \ldots \ldots \ldots \ldots .41$

3.8.4 Stop of Parameter Monitoring $\ldots \ldots \ldots \ldots \ldots \ldots \ldots, 41$

3.9 Daily Operational Readiness Test $\ldots \ldots \ldots \ldots \ldots \ldots \ldots \ldots . \ldots 1$

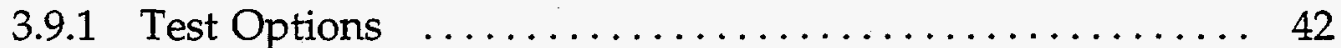

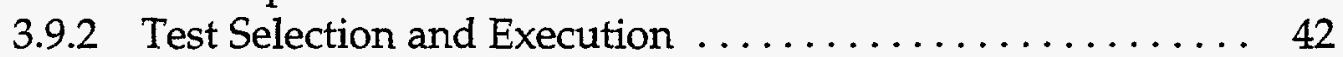

3.9.3 Exiting DORT $\ldots \ldots \ldots \ldots \ldots \ldots \ldots \ldots \ldots \ldots \ldots, 43$

3.10 Startup and Shutdown Sequencing $\ldots \ldots \ldots \ldots \ldots \ldots \ldots, 43$

4. MACINTOSH INSTRUCTOR STATION HARDWARE

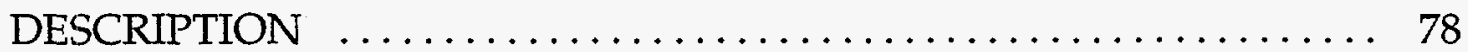

5. ADDITIONAL IMPLEMENTATIONS $\ldots \ldots \ldots \ldots \ldots \ldots \ldots . \ldots . \ldots . \ldots$ 


\section{FIGURES}

Figure 2-1 Host Computer Software Interface Diagram $\ldots \ldots \ldots \ldots \ldots 5$

Figure $3-1$ Simulator Status Summary $\ldots \ldots \ldots \ldots \ldots \ldots \ldots \ldots \ldots$

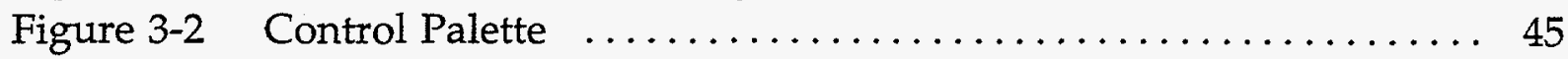

Figure 3-3 Menu Bar with IC Control Menu Selected ............... 46

Figure 3-4 Simulator Backtrack Control Window ............... 47

Figure 3-5 System Malfunction Card $\ldots \ldots \ldots \ldots \ldots \ldots \ldots \ldots \ldots \ldots . \ldots \ldots$

Figure 3-6 Malfunction Selection Window $\ldots \ldots \ldots \ldots \ldots \ldots \ldots \ldots . \ldots \ldots$

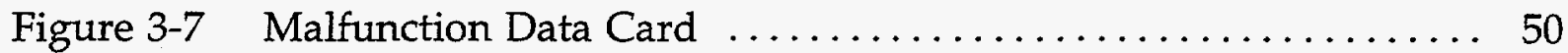

Figure 3-8 Malfunction Description Card .................. 51

Figure 3-9 Malfunction Summary Window .................. 52

Figure 3-10 Malfunction Entry Window for Discrete Malfunction ....... 53

Figure 3-11 Malfunction Entry Window for Variable Malfunction ...... 54

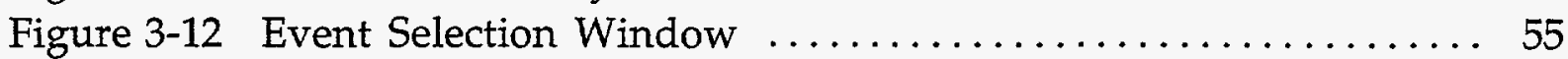

Figure $3-13$ System Remote Function Card $\ldots \ldots \ldots \ldots \ldots \ldots \ldots \ldots \ldots$

Figure 3-14 Remote Function Selection Window ................ 57

Figure 3-15 Remote Function Data Card for Boolean Remote .......... 58

Figure 3-16 Remote Function Data Card for Variable Remote .......... 59

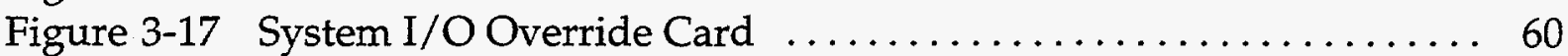

Figure $3-18$ Override Selection Window $\ldots \ldots \ldots \ldots \ldots \ldots \ldots \ldots \ldots \ldots \ldots \ldots \ldots$

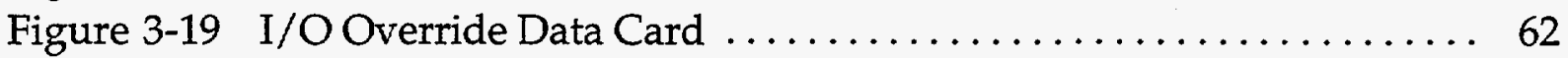

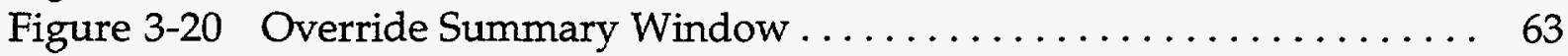

Figure $3-21$ Override Entry Window $\ldots \ldots \ldots \ldots \ldots \ldots \ldots \ldots \ldots \ldots \ldots \ldots \ldots \ldots$

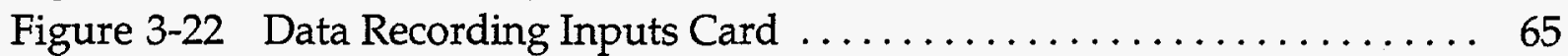

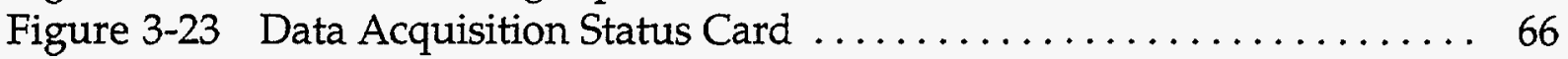

Figure 3-24 Data Acquisition Dialog Box - Recording Complete ........ 67

Figure 3-25 Data Conversion Inputs Card $\ldots \ldots \ldots \ldots \ldots \ldots \ldots \ldots \ldots \ldots$

Figure 3-26 Data Acquisition Error Message $\ldots . \ldots \ldots \ldots \ldots \ldots \ldots . \ldots 69$

Figure 3-27 Data Acquisition Dialog Box - Conversion Complete ....... 70

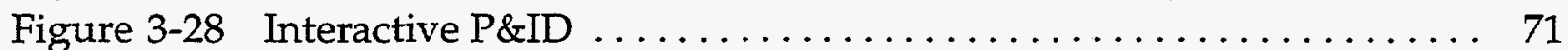

Figure 3-29 Component Data Window - Malfunctions $\ldots \ldots \ldots \ldots \ldots .72$

Figure 3-30 Display Parameter Selection Window $\ldots \ldots \ldots \ldots \ldots \ldots . \ldots 73$

Figure 3-31 Parameter Monitoring Information Window $\ldots \ldots \ldots \ldots \ldots 74$

Figure 3-32 Datapool Variables Chosen Window $\ldots \ldots \ldots \ldots \ldots \ldots \ldots . \ldots \ldots$

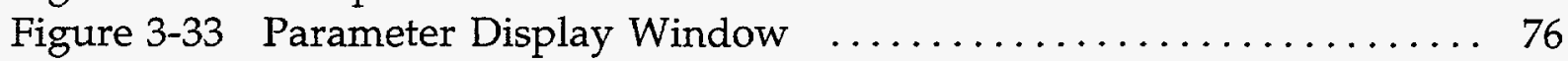

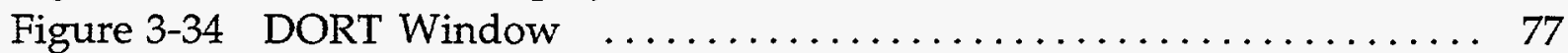




\section{APPENDICES}

APPENDIX A. MACPCM Commands: Messages from Macintosh Instructor Station to Host Computer

APPENDIX B. MACPCM Commands: Messages from Host Computer to Macintosh Instructor Station $\ldots \ldots \ldots \ldots \ldots \ldots$ B-1 


\section{INTRODUCTION}

NRC technical training programs have involved full-scope reactor simulator training from their inception. Initially all simulator training was provided by contracting for time on industry simulators. Over the past several years, in order to insure availability of adequate simulator training time and to allow integration of simulator training into classroom courses, the NRC has contracted to procure and relocate to the Technical Training Center (TTC) in Chattanooga, TN, simulators replicating the General Electric (GE), Westinghouse, Babcock \& Wilcox $(B \& W)$, and Combustion Engineering (CE) reactor designs. These simulators have been purchased through the competitive procurement process from organizations which had previously used them in the conduct of various training programs. The simulation software initially installed on the simulators was developed in the 1970 s or early 1980s, and does not possess sufficient capability or fidelity to meet current requirements for the training of NRC personnel. NRC simulators are used in diverse and demanding programs including training of inspectors, operator license examiners and other technical personnel in transient and accident response, extended scenarios including prolonged operation in Emergency Operating Procedures (EOP's), "what if" (best estimate) analyses, and in support of various human factors and research projects.

\subsection{Instructor Station Upgrade Background}

In 1989, a program to upgrade the GE BWR/6, B\&W and Westinghouse Simulators was begun in order to support the many training needs of the NRC Technical Training Center. This program included the following elements:

(1) Replacement of the Instructor Station on the GE $B W R / 6$ and the B\&W Simulators;

(2) Upgrading each simulator's computer system; and

(3) Installation of a high fidelity thermal hydraulic code to replace the original reactor coolant system thermal hydraulic model on each simulator.

Upgrading the computer system was a prerequisite to installing a high fidelity 
thermal hydraulic code. The Instructor Stations that were originally supplied with the GE BWR/ 6 and B\&W Simulators were each based on a single terminal and a bank of switches. The Instructor Station issued direct input/output (I/O) commands to the terminal controller (as opposed to using an operating system). The controller used was not compatible with newer Encore computers needed to run the improved simulator models. These hardware considerations mandated that the Instructor Station be replaced prior to (or during) the computer system upgrade for the associated simulator.

\subsection{Implementation Choices}

Several factors influenced the method chosen to implement the new Instructor Station:

(1) An instructor station is primarily a human-machine interface to the simulator. It does very little calculation. These types of functions are most appropriately done using microcomputers rather than the super minicomputers that make up the simulator computational engine.

(2) The interface is primarily graphical.

(3) Most actions are the selection of choices (for example, which malfunctions are to be active).

(4) Functions are independent, which means that the best interface is non-modal, whereby any function can be requested at any time. With few exceptions it is not required to enter the commands in a predetermined sequence. This means that the user interface is best implemented using object-oriented programming techniques.

(5) It should be fairly easy to modify an instructor station. Adding a new simulated malfunction should not require large amounts of processing. Because an instructor station is a user interface, the graphical 
presentation of the data should be continually refined as more operating experience is gained. Changing the graphics must be a relatively simple process.

These criteria require a computer with a good graphics engine and support for object-oriented software. The Macintosh ${ }^{\circledR}$ IIcx was the original computer chosen. It has since been replaced by a Macintosh Quadra 700. In the near future, the Quadra will be upgraded to a Power Macintosh ${ }^{\mathrm{TM}}$ class computer.

The Instructor Station Macintosh software was developed using the SuperCard ${ }^{\circledR}$ development tool. SuperCard, published by Allegiant Technologies ${ }^{\mathrm{TM}}$, is an extension to Apple ${ }^{\circledR \prime} S$ HyperCard ${ }^{\circledR}$ tool. SuperCard overcomes several HyperCard limitations, providing full support for multiple, resizable, color windows.

SuperCard uses the SuperTalk programming language. In SuperCard, any object (such as a button, field, card, or window) can have an associated script which is executed as a result of some operator action. SuperTalk is, therefore, an objectoriented programming language.

It should be noted that SuperCard and its SuperTalk language have one significant disadvantage - the script is interpreted at run-time and is not compiled. Applications developed in SuperCard are noticeably slower than compiled applications. However, the speed of the Macintosh Quadra 700 largely compensates for the sluggishness of SuperCard, and it is possible to create a full application in SuperCard in a fraction of the time that more traditional techniques require. 


\section{MACINTOSH INSTRUCTOR STATION SOFTWARE}

The replacement Instructor Station is composed of a user interface, implemented on the Macintosh, and host computer software tasks, implemented on an Encore $32 / 9780$, which respond to the commands issued by the Macintosh, process malfunctions, and update datapool where necessary.

\subsection{Host Computer Software}

The main host computer software task, MACPCM, is a non-base mode program, written in Encore FORTRAN-77 with some Encore Assembly language I/O and file management subroutines. MACPCM is automatically brought into execution during the $32 / 9780$ boot process. It is always running, whether or not the simulator is actually being used.

The major subroutine called by MACPCM is the command handler, CMDH. $\mathrm{CMDH}$ responds to incoming messages from the Macintosh. It is also written in FORTRAN-77.

MALFHAND is the malfunction processing program, and is a non-base mode program written in FORTRAN-77. It executes once per second when the simulator is running. MALFHAND performs the necessary malfunction processing and datapool updates.

Additionally, there are several support routines which are part of the Instructor Station software.

A diagram of the interface between all of the host computer instructor station software programs is presented in Figure 2-1. All of the depicted programs reside on the host computer.

\subsubsection{MACPCM and CMDH}

The principal host task, hereafter referred to as MACPCM, contains a main event loop that is executed every $100 \mathrm{msec}$. The presence of a new Macintosh command is checked for on each pass and, if present, a command handler is called. The command handler (described in more detail later) will, among other things, set some flags that determine the course of processing within the main 


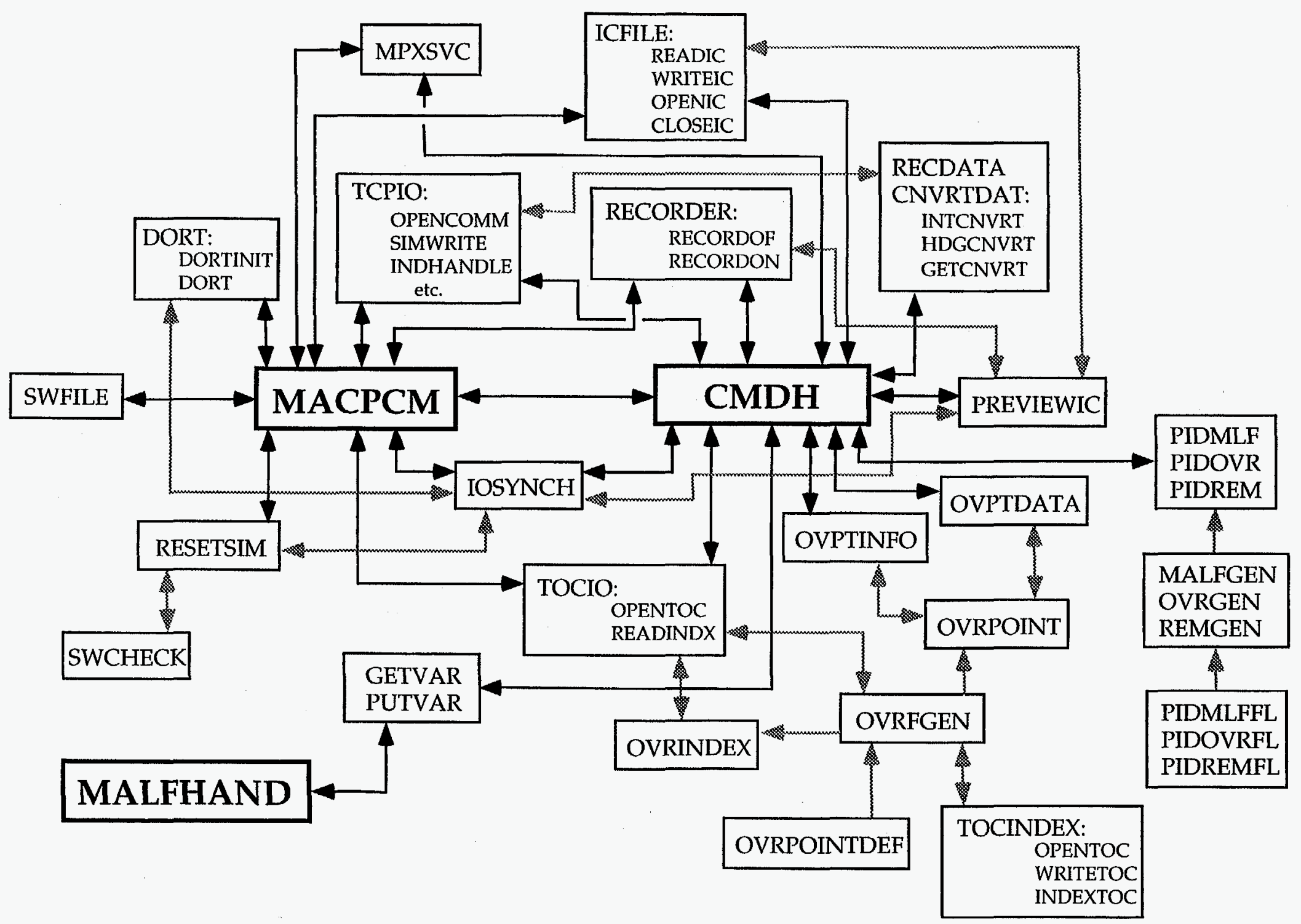

Figure 2-1 Host Computer Software Interface Diagram 
event loop.

The status of the hand-held device (remote control) is checked, which also sets flags which are used in the main loop processing.

If the simulator is in Reset mode (or has just been commanded to go into Reset), the main event loop proceeds through a re-entrant Reset loop. Within this loop, the simulator datapool is initialized from the appropriate records of the disk file(s) which contain the simulator initial conditions. The simulator is then placed in a switch check mode during which all switches that are out of position are flagged by a flashing lamp.

Similarly, if the simulator is in the control benchboard test mode (DORT), the main event loop calls a DORT subroutine that manipulates the simulator I/O buffers - which is seen on the control benchboards as a result of the normal simulator benchboard I/O performed by the host computer.

Special FORTRAN-77 language routines are used to queue the input and output $\mathrm{TCP} / \mathrm{IP} \mathrm{I} / \mathrm{O}$ requests to the Macintosh. All I/O is performed via the MPX-32 ${ }^{\mathrm{TM}} 3 . \mathrm{x}$ operating system, using COMM-32 ${ }^{\mathrm{TM}} \mathrm{TCP} / \mathrm{IP}$ protocols.

The command handler subroutine $(\mathrm{CMDH})$ responds to incoming messages from the Macintosh. Commands are variable in length, with the end marked by a unique character (a tilde, or $\sim$ character). The first element of the command is the function, which will direct processing of the command handler subroutine to the appropriate section. Depending on the function, $\mathrm{CMDH}$ calls minor subroutines to manipulate $\mathrm{I} / \mathrm{O}$ or data files and sets the status of flags which are used by MACPCM.

Table 2-1 lists all of the MACPCM command functions. These can be grouped as follows:

- Placing the simulator in Freeze or Run

- Reset and Snapshot commands;

- DORT commands;

- Malfunction addition, deletion, and status checks;

- Remote function changes and status checks;

- Annunciator control; 
- I/O Override addition, deletion, and status checks;

- Periodic simulator status check (nominally every 2.5 seconds);

- Simulator startup and shutdown commands;

- Backtrack commands;

- Data acquisition commands;

- $\quad$ Piping and Instrument Drawing (P\&ID) data retrieval; and

- Parameter monitoring commands.

Handshaking is built into the MACPCM software where necessary to ensure that a modal sequence is completed before another sequence is started. For example, when a simulator Reset is commanded, there are a number of functions the host software must perform, such as stopping new I/O and saving current status of digital inputs (DI's) and analog inputs (AI's), before acknowledging the Reset to the Macintosh.

\subsubsection{MALFHAND}

A second host task required is the malfunction handling program (MALFHAND). This task is executed once every second.

MALFHAND performs the following functions:

- Countdown of malfunction time delays.

- Countdown of the time limit for a malfunction to be active.

- Computation of malfunction ramp rate.

- Computation of ramped malfunction severity.

- $\quad$ Setting/clearing of malfunction status variables in datapool.

The following activities are also performed by MALFHAND:

- Update of datapool to reflect the value of a malfunction severity.

- Check on the status of malfunction activation buttons on the simulator remote control box.

- Update of the status of specified events which may be used as malfunction activation triggers. 


\subsubsection{Support Programs/Subroutines}

The following is a brief description of the support programs/subroutines which are part of the Instructor Station software:

CNVRTDAT: This is the program for converting the data collected using RECDATA from binary floating point to ASCII format, and then transferring that ASCII data to a Microsoft ${ }^{\circledR}$ Excel spreadsheet file on the Macintosh. It consists of three routines, all written in FORTRAN-77:

(1) INTCNVRT - opens the data file.

(2) HDGCNVRT - transfers headings.

(3) GETCNVRT - transfers file data.

DORT:

This is the program for performing the Daily Operational Readiness Test on the simulator, and it consists of two routines, DORT and DORTINIT. It is written in Assembly.

ICFILE: $\quad$ This is an Assembly program which provides access to the simulator data files for each of the simulator initial conditions. It contains four routines: OPENIC, CLOSEIC, READIC, and WRITEIC.

IOSYNCH: IOSYNCH causes a hard loop until the "I/O in progress" bit is reset. It is written in Assembly.

MALFGEN: This task generates the malfunction database needed for the P\&ID's, placing the data into the random-access, binary file PIDMLF. Its source file is PIDMLFFL, which is written in FORTRAN-77.

OVPTDATA: This task is called by $\mathrm{CMDH}$, and it takes the I/O override command and manipulates the contents of the override tag and value tables in response. It is written in Assembly. 
OVPTINFO: $\quad$ This task is called by CMDH in response to a request for information about a given $\mathrm{I} / \mathrm{O}$ override. It analyzes the 12-word data from the file OVRPOINT and determines what the override can do. OVTPTINFO is written in FORTRAN-77.

OVRFGEN: This program generates the data needed for $\mathrm{I} / \mathrm{O}$ overrides, placing the data into disk files used by MACPCM. It is written in FORTRAN-77. Its input is the point definitions contained in the point description file OVRPOINTDEF, and it generates two files:

(1) OVRPOINT - a random-access file containing the 12 words of override data needed at run time.

(2) OVRINDEX - an alphanumerically sorted index file used for rapidly finding the record associated with a given override.

OVRGEN: $\quad$ This task generates the I/O override database needed for the P\&ID's, placing the data into the random-access, binary file PIDOVR. Its source file is PIDOVRFL, which is written in FORTRAN-77.

PREVIEWIC: This is a subroutine internal to $\mathrm{CMDH}$ which reads the contents of a backtrack IC into memory. It is written in FORTRAN-77.

RECDATA: This is the program for recording specified simulator data in binary floating point format into a file in directory SIMDATA. It is written in FORTRAN-77.

RECORDER: This consists of two Assembly routines, RECORDON and RECORDOF, which turn power to the simulator panel chart recorders on and off, respectively. 
REMGEN:

This task generates the remote function database needed for the P\&ID's, placing the data into the random-access, binary file PIDREM. Its source file is PIDREMFL, which is written in FORTRAN-77.

RESETSIM:

This is a subroutine internal to the MACPCM program which performs the actual reset and switch check process. It is written in FORTRAN-77.

SEVUPDTE: This is a FORTRAN-77 subroutine internal to the MALFHAND program which updates variable malfunction severity values and ramp rates.

SWCHECK: This is an Assembly routine called by RESETSIM which lights the appropriate lamps for DI's and AI's out of position.

SWFILE: $\quad$ SWFILE is written in FORTRAN-77, and creates a disc file that tells (1) which lamp output (LO) should be lit if a given DI is incorrectly set after an initialization, and (2) which LO should be lit and which AI should be set if an AI is mispositioned after an initialization.

TCPIO: $\quad$ TCPIO serves as the Simulator Instructor Station I/O handler. It is composed of numerous routines, all written in FORTRAN-77:

- ENDACTION - services notifications from the CPI.

- GETINDICATION - issues a get indication request.

- INDHANDLE - handles TCP/IP indications.

- OPENCOMM - issues a TCP passive open and then waits until a connection is established.

- SIMWRITE - handles TCP/IP write requests. 
- TCPABRT - issues an abort request.

- TCPCLOS - issues a close request.

- TCPOPEN - opens a TCP connection.

- TCPSEND - issues a send request.

- TCPSETCO - issues a set configuration request.

- TCPSTAT - issues a status request.

- TKWAIT - puts the calling task into wait state; this state is terminated by no-wait completion, message interrupt, break interrupt, and by the time specified having elapsed.

- X:IDTOASC - converts a 32-bit internet address into the dot notation format with leading zeroes.

- X:ITOA - converts an integer value to an ASCII string; the ASCII string is right justified.

TOCINDEX: This is called by routine OVRFGEN to create file OVRINDEX. It consists of three Assembly routines:

(1) OPENTOC - opens I/O override index file OVRINDEX.

(2) WRITETOC - creates a buffer.

(3) INDEXTOC - generates the table of contents.

TOCIO:

This is the I/O override table of contents handler. It is composed of two Assembly routines: 
(1) OPENTOC - opens I/O override index file OVRINDEX and reads the table of contents into a specified buffer.

(2) READINDX - reads override index file OVRINDEX.

VARGET: This program contains the Assembly routine GETVAR, which obtains the current value of the datapool variable corresponding to the severity of a variable malfunction.

VARPUT: This program contains the Assembly routine PUTVAR, which stores the current value of the severity of a variable malfunction in the corresponding datapool location.

XMBOOLS: This is a FORTRAN-77 subroutine internal to the MALFHAND program which updates the status of the event triggers.

XMHAND: This is a FORTRAN-77 subroutine internal to the MALFHAND program which updates the status of the malfunction activation buttons on the remote control box.

\subsection{Macintosh Computer Software}

The Macintosh Instructor Station software is built around SuperCard. A few SuperCard concepts must be explained before the structure of the Instructor Station software is discussed.

\subsubsection{SuperCard Concepts}

SuperCard is an object-oriented, scripted, interpreted software system. A SuperCard application consists of an ordered collection of objects, such as buttons, fields, cards, windows, and projects. An application is composed, at the top level, of at least one project, which in turn has at least one window, which has at least one card. In practice, applications almost always have multiple 
windows and cards, and complicated applications may have multiple projects.

Projects, windows, cards, fields, and buttons interact with each other by sending messages. Some messages are initiated by the system in response to user events. For example, clicking on the mouse button causes the messages mouseDown and mouseUp to be sent by the system through the normal message hierarchy. Other messages are sent by explicit command - as in, send MouseUp to button 3 of card "abc" of window "def".

Messages are acted upon by the first handler having the specified message name that is encountered as the message travels through its hierarchy. Using the previous example, button 3 of card "abc" of window "def" would have a handler structured similar to the following:

$$
\begin{aligned}
& \text { on mouseUp } \\
& \text {..(script).. } \\
& \text { end mouseUp }
\end{aligned}
$$

Messages continue through the hierarchy until a handler is found that can respond to the message. If no handler is found, the message is fielded by the core handlers within SuperCard itself. The message hierarchy can be thought of as a stacked layer, with the element closest to the user on top, and SuperCard on the bottom. The hierarchy can be bypassed by specifying an explicit destination.

SuperCard buttons, fields, and graphics are placed in the cards that make up a window. A window can have multiple cards, but only one card of a window is visible at any one time - which makes each card similar in concept to a record in a database file.

Each card has a background and a foreground layer. Cards can share the same background but have unique, individual foreground layers. Thus, common elements (such as page forward and backward buttons) are usually placed in the background.

Putting it together, the message hierarchy is as follows:

(1) Object (button, field, or graphic)

(2) Card background 
(3) Card foreground

(4) Window

(5) Project

(6) SuperCard core handlers.

\subsubsection{SuperCard Implementation}

The Instructor Station was originally implemented as three SuperCard projects. The first project controlled basic simulator functions such as placing the simulator in freeze and run, resetting to an Initial Condition, and manipulating remote functions. The second project was composed of the windows and cards containing the malfunction data. The third project consisted of the windows and cards containing the $\mathrm{I} / \mathrm{O}$ override data. The application was split into three projects for convenience; it kept the size of each project below the capacity of a single 3.5" floppy disk. Because of the availability of tape backup for the Macintosh, the three projects have now been combined into one large project (approximately 5 MBytes in size).

Two windows are always displayed. One window contains a summary of the current simulator state - for instance, which Initial Condition is currently being used. The other window is a control palette, which the instructor uses to place the simulator in run or freeze, and initiate a reset or snapshot.

Other windows are displayed as requested by the instructor. These windows show such things as:

- the current malfunction status;

- a list of initial conditions;

- information specific to each initial condition;

- lists of remote functions, by system and function;

- lists of malfunctions, by system and function; and

- lists of I/O overrides, by system and function.

Still other windows are used for entering simulated malfunctions and remote functions and for activating I/O override. Examples of the Instructor Station windows can be found in Figures 3-1 through 3-33 (the actual windows are in color). 
When the instructor presses a button on one of the windows or initiates an action by pulling down a menu item, messages are sent to the appropriate objects to perform the requested action. Usually, this results in an ASCII message being sent to the host computer via Ethernet. The function is then executed by the host computer software. For example, pressing the Reset pushbutton results in a message being sent to the host that commands the simulator to be reset to the specified initial condition. The host computer then performs a switch check and, finally, resets the simulator database to the commanded initial condition.

Instructors select an action by clicking on a pre-defined set of items. To avoid having to perform extensive error checking of instructor input, there are very few items that are entered on the keyboard. Instead, the instructor clicks on the appropriate item in a list of possible entries. This method significantly reduces the chance of erroneous instructor action.

\subsection{Communication Protocol}

As stated previously, the command handler subroutine on the host computer responds to the incoming messages from the Macintosh. Commands are variable in length, with the end marked by a unique character (a tilde, or character). The first element of the command is the function, which directs processing of the command handler subroutine to the appropriate section.

Appendices $\mathrm{A}$ and $\mathrm{B}$ each contain a list of the MACPCM commands and the associated message contents. Appendix A defines messages from the Macintosh Instructor Station to the host computer. Appendix B defines messages from the host computer to the Macintosh Instructor Station. 
Table 2-1 MACPCM Commands

\section{Command}

\section{Number Purpose}

01

02

04

50

51

52

05

06

07

08

09
Place simulator in RUN

Place simulator in FREEZE

Reset simulator to specified Initial Condition

Read IC contents

Get a line of malfunction data

Get a line of override data

Switchcheck Override

Snapshot simulator to specified Initial Condition

Place simulator in Daily Operational Readiness Test (DORT)

Toggle DORT Run and Freeze modes

Toggle meter/recorder Sweep and Step modes

Initiate specified DORT test

Exit simulator DORT mode

Clear all malfunctions

Modify specified variable rate malfunction

Activate specified boolean malfunction

Delete specified boolean or variable malfunction

Activate specified variable rate malfunction

Make malfunction processing inactive

Make malfunction processing active

Get current severity of specified variable malfunction

Turn remote function monitoring off

Get current status of a string of boolean remote functions

Toggle specified boolean remote function

Get current status of a string of variable remote functions

Change specified variable remote function

Get current status of an individual boolean remote function 
Table 2-1 (continued)

Command

Number

Purpose

26

28

29

30

31

34

35

36

37

38

42

44

45

47

41

46

49

54

48

55

56

53

57
Silence annunciator horn

Issue annunciator acknowledge and reset

Disable annunciator horn

Enable annunciator horn

Get current reactor pressure, power, flow, and core life

Check status of requested I/O override

Activate an I/O override

Delete an I/O override

Cancel I/O override setup

Clear all $\mathrm{I} / \mathrm{O}$ overrides

Get simulator status

Sequence simulator software startup

Sequence simulator software shutdown

Snap a backtrack IC

Preview a backtrack IC

Cancel backtrack

Start data acquisition program

See if data recording program is still active

Activate data conversion program

Transfer data acquisition headings

Get more data acquisition data

Stop data acquisition system

P\&ID data retrieval 
Table 2-1 (continued)

Command

Number Purpose

60

Obtain status of identified variables

61

Add a datapool variable to monitored list

62

Update status of monitored datapool variables

63

Delete a datapool variable from monitored list

64 Clear all monitored datapool variables

98

Retransmit last message 


\section{MACINTOSH INSTRUCTOR STATION CAPABILITIES}

The Macintosh Instructor Station retains all of the functions that were provided with the original Instructor Station. In addition, a data acquisition system, interactive P\&ID's, and a parameter monitoring feature have been added to the capabilities of both the GE BWR/ 6 and B\&W Instructor Stations. Also, I/O override has been added to the GE BWR/6 Instructor Station. (The original $B \& W$ Instructor Station included I/O override and that capability was retained in the new B\&W Instructor Station.)

The examples given in the following description and the referenced figures are all based on the GE BWR/ 6 Simulator Instructor Station.

\subsection{Main Screen}

The main screen is always displayed on the Macintosh monitor when the Instructor Station is in operation. It consists of the Simulator Status Summary, the red Control Palette, from which the simulator can be controlled, and the Menu Bar with its eight options.

\subsubsection{Simulator Status Summary (Figure 3-1)}

This window contains current information on the status of the simulator: the number of the Initial Condition (IC) currently selected, the number of malfunctions and I/O overrides currently active, the number of the IC to which the simulator will be reset, and the number of the IC to which the current conditions will be snapshot (if desired). Also displayed is the current real time and the amount of time the current IC has been running since last resetting the simulator.

\subsubsection{Control Palette (Figure 3-2)}

The Control Palette is a vertical red box containing eight (8) buttons which are used to control the simulator:

Freeze/Run

Reset

Snapshot 


\section{Malf Inactive \\ BackTrack \\ Ann Horn Off \\ Ann Silence \\ Ann Ack/Reset.}

\subsubsection{Menu Bar (Figure 3-3)}

The menu bar is used to access the different features of the Instructor Station. There are eight (8) menus from which to select:

File

IC Control

Malfunctions

Remotes

I/O Overrides
Contains four items: Print, DORT, Close Windows, and Quit.

Provides access to information about the simulator IC's and provides the means to enter or change the reset and snapshot IC numbers; also provides another means of placing the simulator in and out of "freeze".

Used to activate and delete malfunctions; also provides access to a summary of active malfunctions and to a list of all available malfunctions.

Used to activate and deactivate remote functions; also provides access to a list of all available remote functions.

Used to activate and delete I/O overrides; also provides access to a summary of active overrides and to a list of all available overrides.

Data Acquisition Used to activate the data recording process and the data conversion and transfer processes of the data acquisition feature. 
P\&ID's

Parameter Monitoring $\begin{aligned} & \text { Used to allow monitoring of selected } \\ & \text { datapool variables. }\end{aligned}$
Used to display interactive P\&ID's from which malfunctions, remote functions, and $\mathrm{I} / \mathrm{O}$ overrides for individual components can be accessed.

\subsection{Simulator Initialization}

The instructor may perform the following functions:

(1) Initiate a snapshot of a particular condition

(2) Initialize the simulator to a particular condition.

(3) Freeze and restart simulation.

(4) Re-initialize the simulator to a point in time (up to 60 minutes) since the last reset.

\subsubsection{Snapshot (with protect feature)}

Snapshot of a particular condition to an initial condition set is controlled by the "Snapshot" command which can be issued four different ways:

(1) Select the Snapshot button on the Control Palette, or

(2) Select "Snapshot" from the IC Control menu, or

(3) Press Command-S on the keyboard, or

(4) Use the remote control box.

The software system provides for a minimum of 72 initial condition sets. Of these, 48 are considered permanent initial condition sets and are provided with a protect feature for snapshot. This protect feature for the first 48 initial conditions (IC's) involves entering a password when prompted by the Instructor Station software. If no password or an incorrect one is entered, an appropriate message is displayed and the snapshot to a protected IC is not allowed.

If no IC number is specified for the target snapshot, the snapshot will go to the default IC, number 72. Prior to the snapshot, a prompt asks for confirmation of the default snapshot as the target snapshot. If this is not desired, the snapshot 
command may be canceled at this point and an IC number may then be specified.

After a snapshot, the data stored and displayed for the IC will be updated on the corresponding IC Specific Data window. The description of the IC may be changed by typing the desired information in the Comments box of the window. When the window is closed, a message will appear asking for confirmation of the change request. If the changed comment is for a protected IC, a prompt will request the password. If no password or an incorrect one is entered, an appropriate message is displayed and the change is not allowed.

\subsubsection{Initialization}

Initialization is controlled via the "Reset" command which can be issued four different ways:

(1) Select the Reset button on the Control Palette, or

(2) Select "Reset" from the IC Control menu, or

(3) Press Command-R on the keyboard, or

(4) Use the remote control box.

When reset is commanded, a request for confirmation is made to ensure this is the desired action. Upon confirmation, the simulator enters switchcheck, the Reset button on the Control Palette is highlighted yellow, and the Simulator Reset Control window appears.

In switchcheck, the simulator control panel status is compared to that dictated by the selected IC. Any and all control switches which are improperly set cause an indicator lamp in the vicinity of each misaligned switch to flash. The control conditions are continuously scanned, and each indicator lamp is extinguished as the corresponding control switch is placed to the correct position. Additionally, for each continuous control (potentiometer), a nearby meter is driven to continuously indicate the magnitude and direction of misalignment and an annunciator window flashes. The meter is downscale and the annunciator extinguishes when the potentiometer output is at the correct value for the IC. When all controls are properly aligned, all appropriate control board indications for the new IC are presented and the simulator is placed in freeze (i.e., the simulator will "fall through" switchcheck). 
The Simulator Reset Control window (which opened when the simulator entered switchcheck) allows override of the switchcheck process. This window automatically closes if the simulator falls through switchcheck or if its Override button is selected (which forces the simulator to fall through switchcheck).

\subsubsection{Freeze}

The simulator may be placed in and out of "freeze" by:

(1) Selecting the "Freeze/Run" item from the IC Control menu, or

(2) Pressing Command-F on the keyboard, or

(3) Selecting the Freeze/Run button on the Control Palette.

When the simulator is in freeze, the Freeze/Run button on the Control Palette will be highlighted yellow. When the simulator is running, the button will be white.

\subsubsection{Backtrack}

Current simulator conditions are snapshot every two (2) minutes while the simulator is running. Thirty of these snapshots are retained, equivalent to 60 minutes of run time. The backtrack IC's are non-volatile, as are the other IC's, meaning they will not be lost when the simulator is reset or shut down.

The instructor may choose to initialize the simulator using any of the available backtrack IC's. To issue a Backtrack command, the simulator must first be placed in Freeze, which enables the Backtrack button on the Control Palette. A Backtrack command is issued by selecting the Backtrack button, which highlights yellow and causes the Simulator Backtrack Control window (Figure 3-4) to appear. The available backtrack IC's, along with the date and time they were made, are listed in a scrollable window. The desired backtrack IC is then selected by clicking on the corresponding line.

If it is desired to preview the selected IC, the "Preview" button is selected. All simulator indicators will reflect the IC's conditions. If it is desired to reset to the selected IC, select the "Reset" button. The simulator enters into switchcheck for the selected backtrack IC. If it is desired to exit Backtrack, click on the "Cancel" button. Simulator conditions return to those existing at the time of selecting 
Backtrack.

\subsection{Malfunction Control}

The Instructor Station provides access to numerous malfunctions, which cause a variety of failures in the simulated systems. The malfunctions are arranged and numbered by system 2- or 3-letter designator. As an example, the RHR Pump Trip malfunction on the GE Simulator is numbered E12-1; "E12" is the system designator for the Residual Heat Removal System.

A maximum of twenty (20) malfunctions can be activated at a time. If an attempt is made to activate more than 20 , an appropriate message will be received and the action will not be allowed.

If the Malfunction Inactive feature is selected, as indicated by a yellow "Malf Inactive" button on the Control Palette, the timers for any time-delayed malfunctions will stop. If any new malfunctions are added while this feature is selected, they will not be effective until the feature is deselected. Any active malfunctions will still be in effect. However, if an active malfunction has a limit on the time it will be active, the associated timer will stop while this feature is selected.

Each malfunction is identified by a unique number. If a malfunction has options, each option has a unique number. For example, the RHR Pump Trip malfunction, E12-1, has four options: pump A, pump B, pump C, or all pumps. Each option has a unique number.

When the instructor activates a malfunction, the Macintosh sends the appropriate message to the host computer. This message specifies which malfunction is to be activated; whether it is discrete (boolean) or variable; if variable, the magnitude and ramp rate along with an array pointer; any time delay before activation; any desired length of time for the malfunction to be active; and any activation trigger.

Taking into account any specified time delay, activation trigger, ramp rate, and/or activation time, the host computer then sets the malfunction active in datapool. If the malfunction is a variable one, the host computer program MALFHAND uses the array pointer received from the Macintosh to transfer the 
specified magnitude (after performing any necessary conversion) to the appropriate datapool variable representing the malfunction severity.

\subsubsection{Available Malfunctions}

A list of those systems for which there are malfunctions can be accessed through the hierarchical menu item "Select Malf System/Category" found in the Malfunctions menu.

A list of the malfunctions for a particular system can be obtained by selecting the corresponding menu item. For example, clicking on the item "RHR" will open the system malfunction card shown in Figure 3-5, which is a list of the malfunctions available for the Residual Heat Removal System (RHR).

The system malfunction cards are arranged in alphabetical order and the pointing hands in the lower corners of the cards allow movement from one card to the next without having to return to the hierarchical menu. For example, referring to Figure 3-5, clicking on the hand in the lower left corner opens the card for the Reactor Water Cleanup System; clicking on the hand in the lower right corner opens the card for the Rod Control \& Information System. These cards are before and after, respectively, the one for the Residual Heat Removal System.

\subsubsection{Individual Malfunction Data}

The data for a particular malfunction can be accessed by selecting the malfunction of interest on either the system malfunction card or the scrollable Malfunction Selection window (Figure 3-6). An example of the malfunction data card which appears is shown in Figure 3-7.

A description of the cause and effects of the malfunction can be obtained by clicking on the "Description" button at the bottom of the malfunction data card. The malfunction description card (an example is shown in Figure 3-8) also indicates any available options, as well as whether the malfunction is discrete or variable.

The malfunction data cards and malfunction description cards are arranged in alphabetical order by malfunction number and the pointing hands in the lower 
corners of the cards allow movement from one card to the next without having to return to the system malfunction window. For example, referring to Figure 37, clicking on the hand in the lower left corner opens the data card for malfunction E12-2, RHR Steam Reducing Valve Failure; clicking on the hand in the lower right corner opens the one for malfunction E21-1, LPCS Pump Trip. These data cards are before and after, respectively, the one for malfunction E12-3.

\subsubsection{List of Activated Malfunctions}

To obtain a list of malfunctions which have been activated, select "List Active Malfunctions" from the Malfunctions menu, or press Command-M. The Malfunction Summary window (Figure 3-9) will appear.

As well as providing a list of all activated malfunctions and their status, the Malfunction Summary also displays the following:

(1) The severity level of a variable malfunction. If the severity is being ramped to the desired value, this will be reflected in the value displayed.

(2) The time remaining before a time-delayed malfunction becomes active. If more than a minute remains, the remaining time is shown in minutes; if less than a minute remains, the remaining time is counted down in seconds.

(3) The time remaining before a malfunction is automatically deleted if such a length of time was specified when the malfunction was activated.

A malfunction is shown to be in one of the following states on the Malfunction Summary:

(1) Active: The malfunction is active on the simulator.

(2) Pending: The malfunction is not active on the simulator either because a) it is keyed to a trigger (remote control box or event) which has not activated, 
b) it was entered while the simulator was in Freeze and the simulator is still in Freeze, or c) it was entered while Malf Inactive was selected and Malf Inactive is still selected.

(3) Timing: The malfunction is not active on the simulator because it was entered with a time delay which has not timed out.

(4) Ramping: The malfunction is active on the simulator and its severity is ramping up/down over a specified period of time to the desired value.

The Malfunction Summary window is updated every three seconds.

\subsubsection{Malfunction Activation and Change}

To activate a malfunction, click on the radio button next to the malfunction number on the malfunction data card (Figure 3-7), which opens the corresponding Malfunction Entry window. (An example of this window is provided in Figure 3-10 for a discrete malfunction and in Figure 3-11 for a variable malfunction.)

When activating a variable malfunction, enter the desired severity by either 1) clicking on the "Set" button and inserting the desired severity at the prompt, or 2) clicking on the "Up" and "Down" arrows until the desired value is reached (one click will change the severity by $2 \%$ of its range). The acceptable range for the value is specified on the Malfunction Entry window (Figure 3-11).

If the severity of a variable malfunction is to ramp over a period of time to the entered value, click on the "Yes" button. Enter the desired ramp time, in seconds, at the prompt.

If there is to be a time delay before activation, click on "Yes" and enter the desired time delay, in minutes, at the prompt. If the malfunction is to be active for a specific time, click on "Yes" and enter the desired length of time, in minutes, at the prompt. (Tenths of minutes are acceptable in both instances.) 
If the malfunction is to be activated by the remote control box, click on the "Remote" button and enter the number of the desired remote control button at the prompt. Alternatively, if it is to be activated by an event, click on the "Event" button. The Event Selection window (Figure 3-12) will appear. Select the desired event, enter any necessary values in response to prompts received, and click on the "OK" button.

Click on the "Activate" button after making the above selections, or click on the "Cancel" button to exit without activating the malfunction. Either action will close the Malfunction Entry window.

The status of the malfunction on the malfunction data card (Figure 3-7) will change from "Inactive" to "Active".

Note that until the malfunction has been actuated by clicking on the "Activate" button, the "Delete" button on the Malfunction Entry card is disabled. When the "Delete" button is enabled, the "Activate" button is disabled.

To change the severity of a variable malfunction, open the Malfunction Entry window and enter the new value for the severity. Click on the "Change" button after entering the new value, or click on the "Cancel" button to exit without changing the malfunction. Either action will close the Malfunction Entry window. The status of the malfunction on the Malfunction Summary (Figure 39) will change to reflect the new severity value.

\subsubsection{Malfunction Deletion}

To delete an activated malfunction, open the Malfunction Entry window (Figure 3-11) and click on the "Delete" button, or click on the "Cancel" button to exit without deleting the malfunction. Either action will close the Malfunction Entry window.

If it is desired to delete all active malfunctions, select "Clear All Malfunctions" from the Malfunctions menu. A request for confirmation is made prior to the deletion to ensure this is the desired action.

The Malfunction Summary (Figure 3-9) will update to reflect the malfunction deletion(s). 


\subsection{Remote Function Control}

Each Instructor Station has numerous remote functions, which provide access to simulated controls which are not on the control room panels, and also allow changing of certain plant parameters and control circuits. The remote functions are arranged and numbered by system 2- or 3-letter designator. As an example, the remote function for the RR Pump Lockout Relay on the GE Simulator is numbered B33-1; "B33" is the system designator for the Reactor Recirculation System.

Each remote function is identified by a unique number. If a remote function has options, each option has a unique number. For example, the Shutdown Cooling Isolation Bypass remote function, E12-4, has two options: E12-F053A and E12F053B. Each option has a unique number.

When the instructor activates a remote function, the Macintosh sends the appropriate message to the host computer. This message specifies which remote function is to be activated; whether it is discrete (boolean) or variable; and, if variable, the magnitude and an array pointer.

The host computer then sets the remote function status in datapool. If the remote function is a variable one, the host computer transfers the magnitude received to the correct location in an array table. The array table contains the datapool variables associated with the variable remote functions.

\subsubsection{Available Remote Functions}

A list of those systems for which there are remote functions can be accessed through the hierarchical menu item "Select Remote System/Category" found in the Remotes menu.

A list of the remote functions for a particular system can be obtained by selecting the corresponding menu item. For example, clicking on the item "CRDH" will open the system remote function card shown in Figure 3-13, which is a list of the remote functions available for the Control Rod Drive Hydraulics System (CRDH).

The system remote function cards are arranged in alphabetical order and the 
pointing hands in the lower corners of the cards allow movement from one card to the next without having to return to the hierarchical menu. For example, referring to Figure 3-13, clicking on the hand in the lower left corner opens the card for the Condensate System; clicking on the hand in the lower right corner opens the card for the Containment. These cards are before and after, respectively, the one for the CRDH System.

\subsubsection{Individual Remote Function Data}

The data for a particular remote function can be accessed by selecting the remote function of interest on either the system remote function card or the scrollable Remote Function Selection window (Figure 3-14). An example of the remote function data card which will appear is shown in Figure 3-15. Remote function C11-2 is a boolean remote function. The status column shows the two possible states for the remote function. The current state is indicated two ways: an " $X$ " in its box and the label is highlighted yellow.

If the remote function is a variable one, its data card will look like the one shown in Figure 3-16. The variable remote's range is indicated in brackets, with the units in parentheses, in the item column. Its current status is shown in the status column.

The remote function data cards are arranged in alphabetical order by remote function number and the pointing hands in the lower corners of the cards allow movement from one card to the next without having to return to the system remote function window. For example, referring to Figure 3-16, clicking on the hand in the lower left corner opens the data card for remote function B33-2, Endof-Cycle RPT Bypass; clicking on the hand in the lower right corner opens the one for remote function C11-2, CRDH FCV Selection. These data cards are before and after, respectively, the one for remote function C11-1.

\subsubsection{Remote Function Operation}

To change the state of a remote function, click on the radio button next to the remote function number on the remote function data card (Figure 3-16). If it is a boolean remote, the state will change in the status column. If it is a variable remote, enter the desired value at the prompt. The new value will then be shown in the status column. 


\subsection{I/O Override Control}

The I/O Override feature allows the instructor to command the simulator to override the calculated indications on some meters, recorders, and controllers, and to ignore student manipulation of some switches and controllers. For output, the instructor can fail a meter or recorder to a specified reading, or to the condition that exists at the time that the override is activated. For input, the instructor can specify that a given switch or controller is to fail to the present condition. The overrides are arranged and numbered by system 2- or 3-letter designator. As an example, the override for the Control Rod Drive Pump Control Switches is numbered C11-1; "C11" is the system designator for the Control Rod Drive Hydraulics System.

A maximum of twenty (20) overrides can be activated at a time. If an attempt is made to activate more than 20 , an appropriate message will be displayed and the action will not be allowed.

When the instructor initiates an override, the Macintosh sends the appropriate message to the host computer, specifying which override is to be activated and the override condition. The host computer accesses its database of available overrides and generates the proper entries in special override memory tables. The memory tables are used by the executive system's override subroutine to place the commanded override into effect. Up to twenty devices can be overridden at any time, with a single device having up to twelve $\mathrm{I} / \mathrm{O}$ elements (for example, a controller could have several pushbuttons, at least one meter, and several lamps that are simultaneously overridden).

The host computer database is generated using a special set of host utility software. The software consists of a source file specifying each override by name and a list of the $1 / O$ devices that the override affects, and a utility program that automatically generates an indexed, sorted database from the source file.

To implement I/O Override for output, an override subroutine is called immediately prior to issuing control benchboard I/O commands. The override subroutine copies the output buffers used by the models to a local I/O buffer, modifying the local buffer as required by I/O Override. The I/O initiation commands are then issued. 
Input from the control benchboards is directed to a local buffer. When the I/O operation completes, an I/O end-action routine copies the local buffers to the I/O buffers seen by the models and then calls the override subroutine to apply any input overrides.

In essence, then, the control benchboard I/O data is contained in two buffers one seen by the models, and another seen by the hardware, differing only to the extent commanded by I/O Override.

\subsubsection{Available I/O Overrides}

A list of those systems for which there are overrides can be accessed through the hierarchical menu item "Select Override System/Category" found in the I/O Overrides menu.

A list of the overrides for a particular system can be obtained by selecting the corresponding menu item. For example, clicking on the item "FW Control" will open the system I/O override card shown in Figure 3-17, which is a list of the overrides available for the Feedwater Control System.

The system I/O override cards are arranged in alphabetical order and the pointing hands in the lower corners of the cards allow movement from one card to the next without having to return to the hierarchical menu. For example, referring to Figure 3-17, clicking on the hand in the lower left corner opens the card for the Feedwater System; clicking on the hand in the lower right corner opens the card for the Generator. These cards are before and after, respectively, the one for the Feedwater Control System.

\subsubsection{Individual I/O Override Data}

The data for a particular I/O override can be accessed by selecting the $1 / O$ override of interest on either the system I/O override card or the scrollable Override Selection window (Figure 3-18). An example of the I/O override data card which will appear for any override is shown in Figure 3-19.

The I/O override data cards are arranged in alphabetical order by override number and the pointing hands in the lower corners of the cards allow movement from one card to the next without having to return to the system $\mathrm{I} / \mathrm{O}$ 
override window. For example, referring to Figure 3-19, clicking on the hand in the lower left corner opens the data card for override C34-4, 1/3 Element Control \& Level A/B Select Switches; clicking on the hand in the lower right corner opens the one for override C41-1, SLC Pump Control Switches. These data cards are before and after, respectively, the one for override C34-5.

\subsubsection{List of Active Overrides}

To obtain a list of I/O overrides which are active, select "List Active Overrides" from the I/O Overrides menu. The Override Summary window (Figure 3-20) will appear.

\subsubsection{I/O Override Activation}

To activate any $\mathrm{I} / \mathrm{O}$ override, click on the radio button next to the override number on the override data card (Figure 3-19), which opens the corresponding Override Entry window (Figure 3-21). Click on the radio button next to the desired option. Not all options are available for each override. Those options which are not available are disabled. If the "Fail to Value" option is selected, enter the desired value at the prompt.

Click on the "Activate" button after making the above selections, or click on the "Cancel" button to exit without activating the override. Either action will close the Override Entry window.

The status of the override on the override data card (Figure 3-19) will change from "Not Overridden" to "Overridden".

Note that until the override has been actuated by clicking on the "Activate" button, the "Delete" button on the Override Entry card is disabled. When the "Delete" button is enabled, the "Activate" button is disabled.

\subsubsection{I/O Override Deletion}

To delete an active I/O override, open the Override Entry window and click on the "Delete" button, or click on the "Cancel" button to exit without deleting the override. Either action will close the Override Entry window. 
If it is desired to delete all active overrides, select "Clear All Overrides" from the I/O Overrides menu. A request for confirmation is made prior to the deletion to ensure this is the desired action.

The Override Summary (Figure 3-20) will update to reflect the override deletion(s).

\subsection{Data Acquisition}

The Data Acquisition feature allows the instructor to collect designated parameter values at a specified interval for a specified period of time during simulator operation. This data can then be converted to ASCII format and transferred to an Excel spreadsheet file for manipulation and plotting. While the Data Acquisition option is in operation, the simulator still retains all aspects of its operability (i.e, it can still be placed in and out of Freeze, malfunctions can be added or deleted, etc.).

This feature is particularly useful for the recording of data, for later analysis and plotting, during transients. Since the data collection interval can be specified, the Data Acquisition feature is also useful during troubleshooting and evaluation of simulator software changes.

A list of the parameters recorded can be obtained by selecting "Parameter List" from the Data Acquisition menu.

The Data Acquisition System consists of two tasks which run on the host computer: RECDATA (data recording task) and CNVRTDAT (data conversion task). There is also an Excel macro which runs on the Macintosh. These programs execute the four operations which must be performed in order to obtain data from instantaneous simulator response and convert it to a continuous set of values inside an Excel spreadsheet ready for plotting. These operations are recording, conversion, transfer, and manipulation.

\subsubsection{Data Recording}

The parameters are recorded at a specified interval by the RECDATA program. The data is saved in binary floating point format to a file in directory SIMDATA on the host computer. The time delay until the commencement of recording 
and the duration of recording are defined by the instructor, as well as the recording interval and the name of the file into which the data will be written. The maximum recording duration is 60 minutes.

To begin data recording, select "Record Data" from the Data Acquisition menu. The Data Recording Inputs card (Figure 3-22) will appear with default values for delay time, recording duration, binary file name, and recording interval specified. The default delay time is zero minutes. The default recording duration is five (5) minutes. The default recording interval is once per second (1 cps). The default filename is "RD" followed by the date and sequence number. The sequence number will change if the default file name is used more than once without restarting the Instructor Station software. For example, if the default name was used twice on September 13, the first file created would have the name RD0913_1 and the second would be RD0913_2.

To change the delay time and/or the recording duration, click on the associated "Up" and "Down" arrows until the desired time is displayed. To change the recording interval, click on the desired time interval. To change the file name, click on the "Change" button and enter the desired name at the prompt. A valid name consists of a maximum of 16 characters; valid characters are letters and numbers.

Click on the "Record" button after making the above selections to begin data recording, or click on the "Cancel" button to exit without recording. Either action will close the Data Recording Inputs card.

If the "Record" button was selected, the Data Acquisition Status card (Figure 3-23) will open. The specified duration and delay times will be displayed, as will the elapsed recording time. The Status card also indicates the simulator mode (Freeze or Run) and the corresponding mode of the data acquisition program (Inactive or Active). Data recording can be started and stopped by placing the simulator in and out of Freeze.

If it is desired to start a data recording session before the specified delay time has elapsed, click on the "Start Now" button. If it is desired to end a data recording session before the specified duration time has elapsed, click on the "Stop Now" button. A request for confirmation is made prior to cessation of recording to ensure this is the desired action. 
When data recording is complete (either because the elapsed recording time equals the specified duration time or because the "Stop Now" button was selected), the Data Acquisition Status card closes and a dialog box (Figure 3-24) appears. If you wish to convert and transfer the collected data to an Excel file now, click on the "Yes" button, which will open the Data Conversion Inputs card; otherwise, click on "No". Either action will close the dialog box.

\subsubsection{Data Conversion and Transfer to Excel}

Since the data is recorded in binary form on the host computer, it must be converted to ASCII format and then exported to an Excel file on the Macintosh for it to be accessible by the instructor. The CNVRTDAT task performs these operations. This task also provides the instructor the opportunity to select which of the recorded parameters will be converted and transferred.

To begin this process, open the Data Conversion Inputs card (Figure 3-25) by either selecting "Convert Data" from the Data Acquisition menu, or clicking on the "Yes" button when the dialog box appears at the completion of data recording.

Click on the Binary File Name "Change" button and enter the name of the file containing the data to be converted. The last filename given when data was recorded will be the default for the binary file name.

Click on the Macintosh File Name "Change" button and enter the name of the file which will contain the converted and transferred data. The default name is the same as the default name for the binary file.

If all parameters are to be converted, click on the "ALL" button. ("ALL" is the default.) Otherwise, click on the "Selected" button to display a list of the available parameters. Select the parameters to be converted by clicking on their associated buttons. After selecting the parameters to be converted, click on the "OK" button, or click on the "Cancel" button. Either action will close the Parameter List.

Click on the "Convert" button after making the above selections to begin conversion and transfer, or click on the "Cancel" button to exit and close the Data Conversion Inputs card. 
If an invalid binary file name was specified prior to selecting the "Convert" button, an error message (Figure 3-26) will be received. Clicking on "OK" will return you to the Data Conversion Inputs card to allow entry of a valid name.

Once all the conversion process inputs have been properly defined, selecting the "Convert" button will begin the conversion and transfer process. A "wait" dialog box will appear telling you to wait while the process is completed.

When data conversion and transfer are complete, the "wait" dialog box closes and another dialog box (Figure 3-27) appears. If you wish to convert and transfer another set of collected data to an Excel file now, click on the "Yes" button, which will close the dialog box and return you to the Data Conversion Inputs card; otherwise, click on "No" which will close the dialog box and the Data Conversion Inputs card.

\subsubsection{Data File Manipulation Using Excel}

The first time a transferred data file is to be opened using Excel, it must be opened using the Excel "OPENDATA" macro. This is to ensure that the file is opened as a comma delimited file and saved as a regular Excel file. OPENDATA will automatically import the data file into an Excel spreadsheet, save a copy of the spreadsheet, and leave the spreadsheet open for use by the instructor.

To open the transferred data file the first time, double-click on the "OPENDATA" icon. (This icon is found in the Sim_Data folder contained in the Excel application on the Macintosh hard disk.)

Type the name of the transferred ASCII file (the Macintosh file name specified prior to beginning the conversion and transfer process) in the dialog box that appears. Click the "OK" button to activate the macro, or the "Cancel" button to exit. Either action will close the dialog box.

An Excel spreadsheet will open and the transferred data will appear. The name of this Excel file will be the specified name appended with ".ASC". The recorded simulator data is now available for manipulation and plotting by the instructor. 


\subsection{Interactive $\mathbf{P} \& I D^{\prime} \mathrm{s}$}

The Interactive P\&ID feature allows the instructor to directly access malfunction, $\mathrm{I} / \mathrm{O}$ override and remote function data for a component shown in a diagrammatic representation of a system. The system diagrams are arranged in alphabetical order.

When the instructor selects a particular component, the Macintosh sends the appropriate message to the host computer, specifying which component (by number) has been selected. (There is a unique number assigned to each component depicted on a diagram.) The host computer then accesses its databases of available malfunctions, I/O overrides, and remote functions. It obtains the data for the specified component and sends that information back to the Macintosh for display.

The host computer databases are generated using a special set of host utility software. The software consists of a source file specifying the malfunctions associated with each component and a utility program that automatically generates a random access, binary file (the database) from the source file. The host software also contains comparable source files and utility programs for the generation of $\mathrm{I} / \mathrm{O}$ override and remote function binary files.

\subsubsection{Available P\&ID's}

A list of available P\&ID's can be accessed through the hierarchical menu item "Select P\&ID" found in the P\&ID's menu.

A P\&ID can be displayed by selecting the corresponding menu item. For example, clicking on the item "Condensate" will open the diagram shown in Figure 3-28, which is the P\&ID for the Condensate System.

\subsubsection{Component Data}

To determine what options are available for a component, click on the associated button. For example, to determine what alternatives exist for Condensate Pump A, click on the button located to the right of the pump. The Options menu (Malfunctions, Overrides, Remotes) will "pop up" to the right of the button, with the appropriate items enabled. In the case of Condensate Pump A, the 
Malfunctions and Overrides menu items would be enabled, while the Remotes menu item would be disabled--there are no remote functions associated with Condensate Pump A.

To obtain a list of component malfunctions, select that item from the Options menu. The corresponding data window will appear (Figure 3-29). To activate a malfunction, click on the radio button next to the malfunction number, which will cause the Malfunction Entry window (Figure 3-10) to appear. From this point, activation proceeds as previously described.

The steps would be similar for obtaining a list of the I/O overrides or the remote functions for a component.

\subsection{Parameter Monitoring}

The Parameter Monitoring feature allows the instructor to monitor selected datapool variables during simulator operation. Only real variables may be monitored; integer and boolean variables cannot be properly displayed.

A maximum of twenty (20) variables can be monitored at a time. If an attempt is made to monitor more than 20 , an appropriate message will be displayed and the additional monitoring will not be allowed.

\subsubsection{Datapool Variable Selection}

To initiate the Parameter Monitoring feature, select the "Select Parameters" item from the Parameter Monitoring menu, which will open the "Display Parameter Selection" window (Figure 3-30). This window contains buttons which are preassigned to specific datapool variables to which access would commonly be desired (e.g., reactor water level and drywell pressure). It also contains a button, labeled "Other", which allows the instructor to enter the name of any real datapool variable for display.

To select pre-assigned variables for display, click on the button(s) associated with the parameters it is desired to monitor.

To select any real datapool variable for display, click on the button labeled "Other". The first time the "Other" button is selected during a monitoring 
session, an information window (Figure 3-31) will appear. After reading it, click on "OK" to continue, or "Cancel" to return to the "Display Parameter Selection" window.

If "OK" was selected, a prompt will ask for the datapool variable name. If the entered name is longer than the maximum eight (8) characters, an appropriate message will be displayed and the name will not be accepted. If a variable name is accepted, the "Datapool Variables Chosen" window (Figure 3-32) will appear. All variable names entered will be displayed in this window so the instructor may know which variables have already been selected.

\subsubsection{Start of Parameter Monitoring}

To begin monitoring, click on the "OK" button on the "Display Parameter Selection" window. Click on the "Cancel" button if it is decided to not begin monitoring.

If "OK" is selected, the "Parameter Display" window (Figure 3-33) will open (after a short time delay) and the "Display Parameter Selection" window and the "Datapool Variables Chosen" window (if open) will close.

Units will be displayed for the selected pre-assigned variables. No units are displayed for the variables which were entered via the keyboard. If an entered variable does not exist, this will be noted on the "Parameter Display" window.

When the instructor starts parameter monitoring by clicking on the "OK" button, the Macintosh sends the appropriate message(s) to the host computer. If pre-assigned variables have been selected, one message contains codes identifying the selected variables. The host computer uses those codes to determine which values from a special parameter value table to return to the Macintosh, after performing any required unit conversion.

If datapool variables names have been entered, a series of messages from the Macintosh to the host computer contains these names (one name per message). The host computer calls a subroutine to retrieve the datapool address for each variable and stores each address in an address table. The address is then used to obtain the variable's value, which is returned to the Macintosh for display. 


\subsubsection{Addition or Deletion of Variables}

Once monitoring has started, variables may be deleted or additional variables may be monitored. Current monitoring will not be affected by either of these activities.

To add another variable to monitoring, select the "Select Parameters" item from the Parameter Monitoring menu. Proceed with selection as described above. When the additional variables have been selected, click on the "OK" button on the "Display Parameter Selection" window.

A variable may be deleted from monitoring using one of three methods:

(1) On the "Parameter Display" window, click on the button associated with the variable to be deleted; or

(2) On the "Display Parameter Selection" window, click on the button associated with the variable to be deleted; or

(3) On the "Datapool Variables Chosen" window, click on the button associated with the variable to be deleted.

\subsubsection{Stop of Parameter Monitoring}

To stop monitoring, close the "Parameter Display" window.

Monitoring may also be stopped by selecting the "Stop Monitoring" item from the Parameter Monitoring menu. A prompt will ask for confirmation. If it is not desired to stop monitoring, the stop command can be canceled at this point and monitoring will continue.

\subsection{Daily Operational Readiness Test}

The Daily Operational Readiness Test (DORT) enables the Simulator Maintenance Staff to test the operational status of the simulator panel hardware. To place the simulator in DORT, select "DORT" from the File menu. The DORT window (Figure 3-34) will appear, and the Control Palette and Simulator Status Summary will close. 


\subsubsection{Test Options}

There is a total of eight tests from which to choose: six individual tests (Meters, Recorders, DI Loop, AI Loop, DI Display, and Lamp Test) and two combined tests (Meters \& Recorders and DI \& AI Loop). The following is a brief description of the individual tests:

Meters: This test checks all meters throughout their operating ranges. Meters are exercised at fixed points of $0,25,50,75$, and $100 \%$ of full scale when operating in the STEP mode. The SWEEP mode causes the indicators to continuously move up and down the face of the meter.

Recorders: This test checks all recorders in a manner identical to the Meter test.

AI Loop: This test allows a manual check of any variable control (potentiometer). Setting a potentiometer to a particular position should result in a corresponding indication on the associated meter.

DI Loop: This test allows a manual check of the panel switches. A certain lamp on a panel section is illuminated at the start of the test. When all the DI's in the section have been set (i.e., all the switches manipulated), the lamp will extinguish.

DI Display: Not currently functional.

Lamp Test: This is an automatic test which sequentially illuminates all simulator lamps.

\subsubsection{Test Selection and Execution}

To select a test, click on the radio button next to the desired test (Figure 3-34). Then take the simulator out of freeze by clicking on the "Freeze" button, which will cause the test to begin. The test being executed may be changed without placing the simulator in freeze by clicking on the radio button for a different test. All tests can be stopped at a given point by clicking on the "Freeze" button. 
When the simulator is initially placed in DORT, the STEP mode for the meter and recorder tests is automatically selected. To change to the SWEEP mode, click on the "Go to Sweep" button. This will result in the lettering on the mode button (right button on the DORT window) changing to "Go to Step". Clicking on the mode button again will change the meter and recorder tests back to the STEP mode.

\subsubsection{Exiting DORT}

To exit DORT and return to normal simulator operation, click on the close box in the upper left corner of the DORT window. The DORT window will close, and the Control Palette and Simulator Status Summary will reappear.

\subsection{Startup and Shutdown Sequencing}

Simulator startup and shutdown are functions of the Macintosh Instructor Station.

The host instructor station software, MACPCM, is automatically brought into execution during the host computer boot process. MACPCM is always running, whether or not the simulator is actually being used.

When an instructor starts the Macintosh Instructor Station, a startup message is sent to the host computer that is fielded by the command handler. In response, MACPCM sends a run request which begins the executive system startup sequence. This process brings all of the simulation software on the host computer into execution.

Similarly, a message is sent to the host computer when the instructor exits the Macintosh Instructor Station. This message, also fielded by the command handler, causes all of the simulator software on the host computer to exit, except for MACPCM.

As a result, the simulator is started up or shutdown without the need for an instructor to log on to the host computer. Instead, the instructor simply starts up the Macintosh; all software is automatically sequenced. The host computer is generally left continuously running. 
Simulator Status Summary

Current IC:

24

Number of Malfunctions:

0

SnapShot IC:

Number of Overrides:

$\mathbf{0}$

Reset IC:

24

Clock Time

Figure 3-1 Simulator Status Summary 


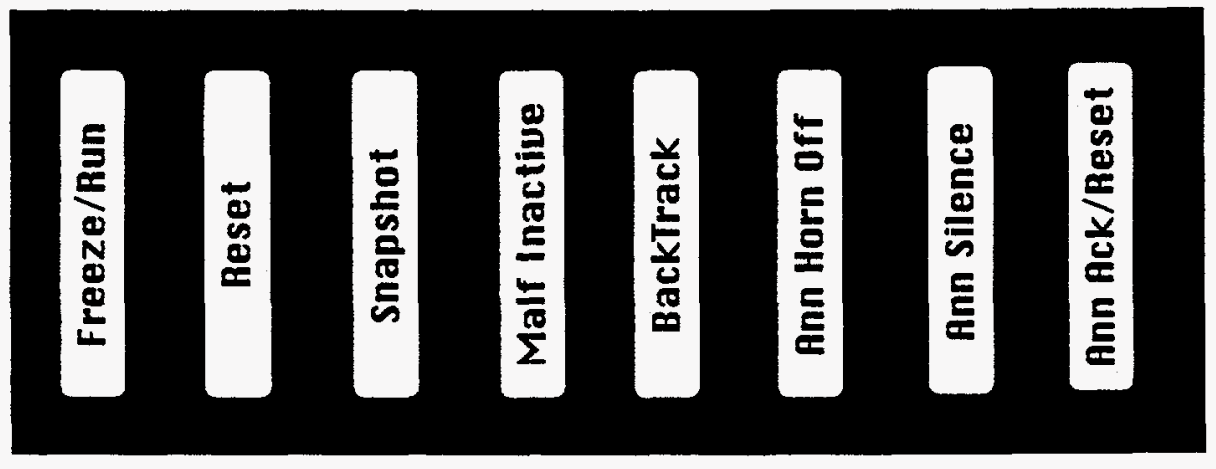

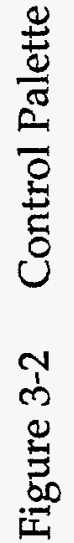




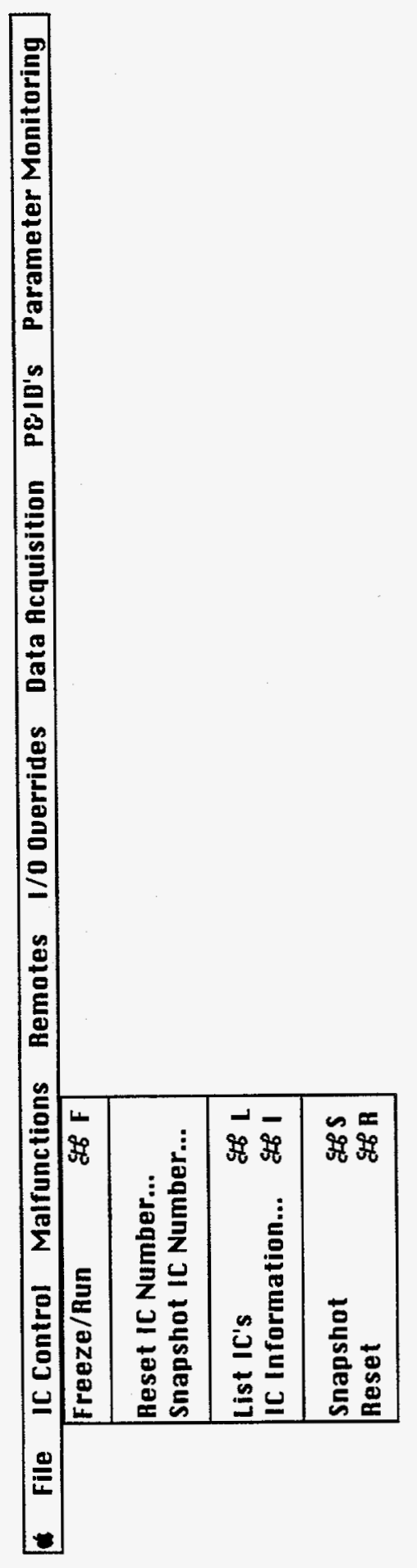




\begin{tabular}{|c|c|c|}
\hline \multicolumn{3}{|c|}{ Simulator Backtrack Control } \\
\hline $\begin{array}{l}74-2 / 23 / 95 \text { 3:48 PM } \\
75-2 / 23 / 95 \text { 3:58 PM } \\
76-2 / 23 / 95 \text { 4:00 PM } \\
77-2 / 23 / 95 \text { 4:10 PM } \\
78-2 / 23 / 95 \text { 4:15 PM } \\
79-2 / 23 / 95 \text { 4:17 PM } \\
80-2 / 23 / 95 \text { 4:19 PM } \\
81-2 / 23 / 95 \text { 4:21 PM } \\
82-2 / 23 / 95 ~ 4: 23 \text { PM }\end{array}$ & 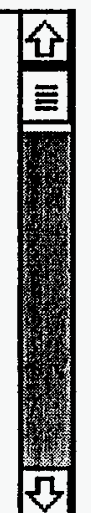 & Cancel \\
\hline
\end{tabular}

Figure 3-4 Simulator Backtrack Control Window 


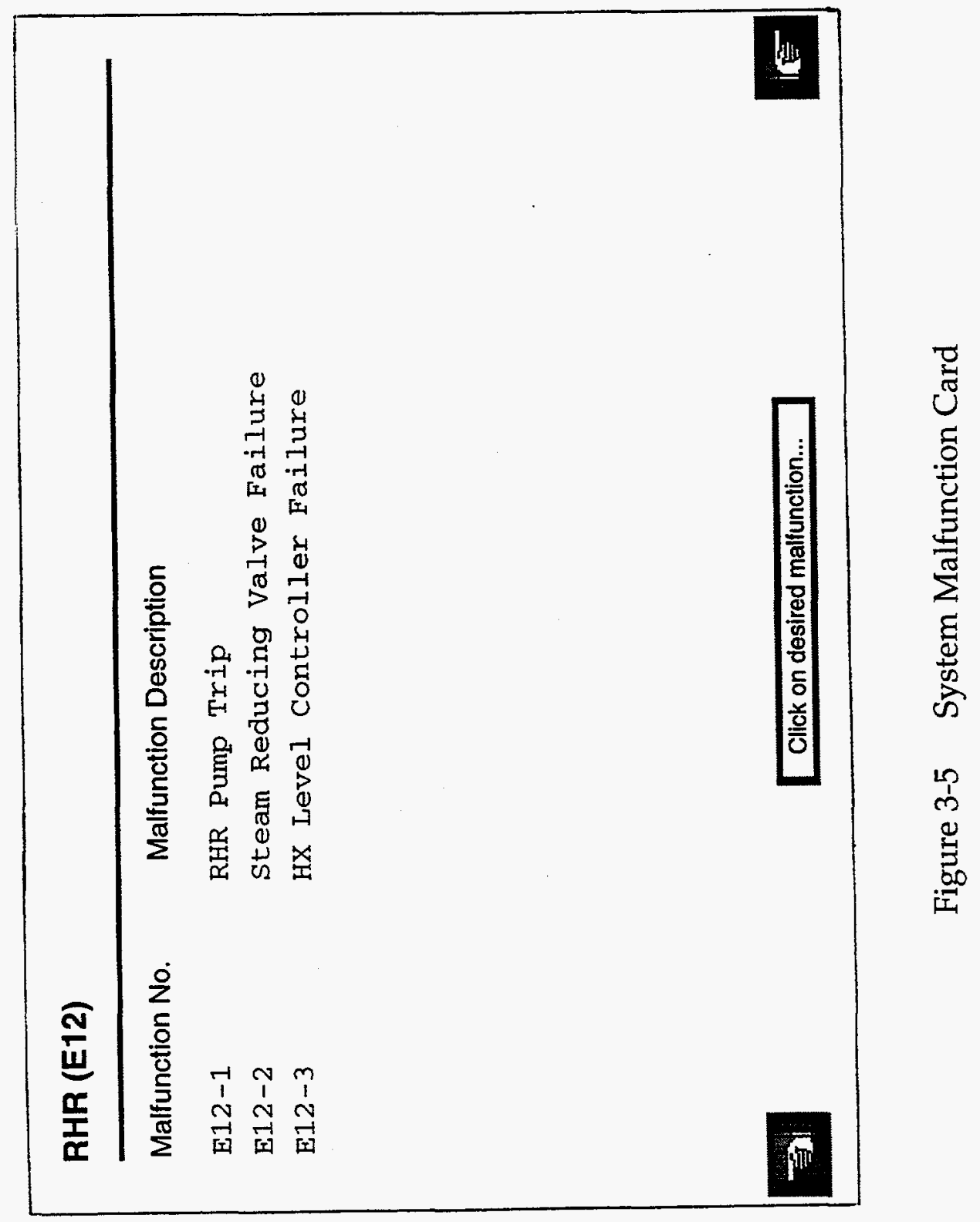




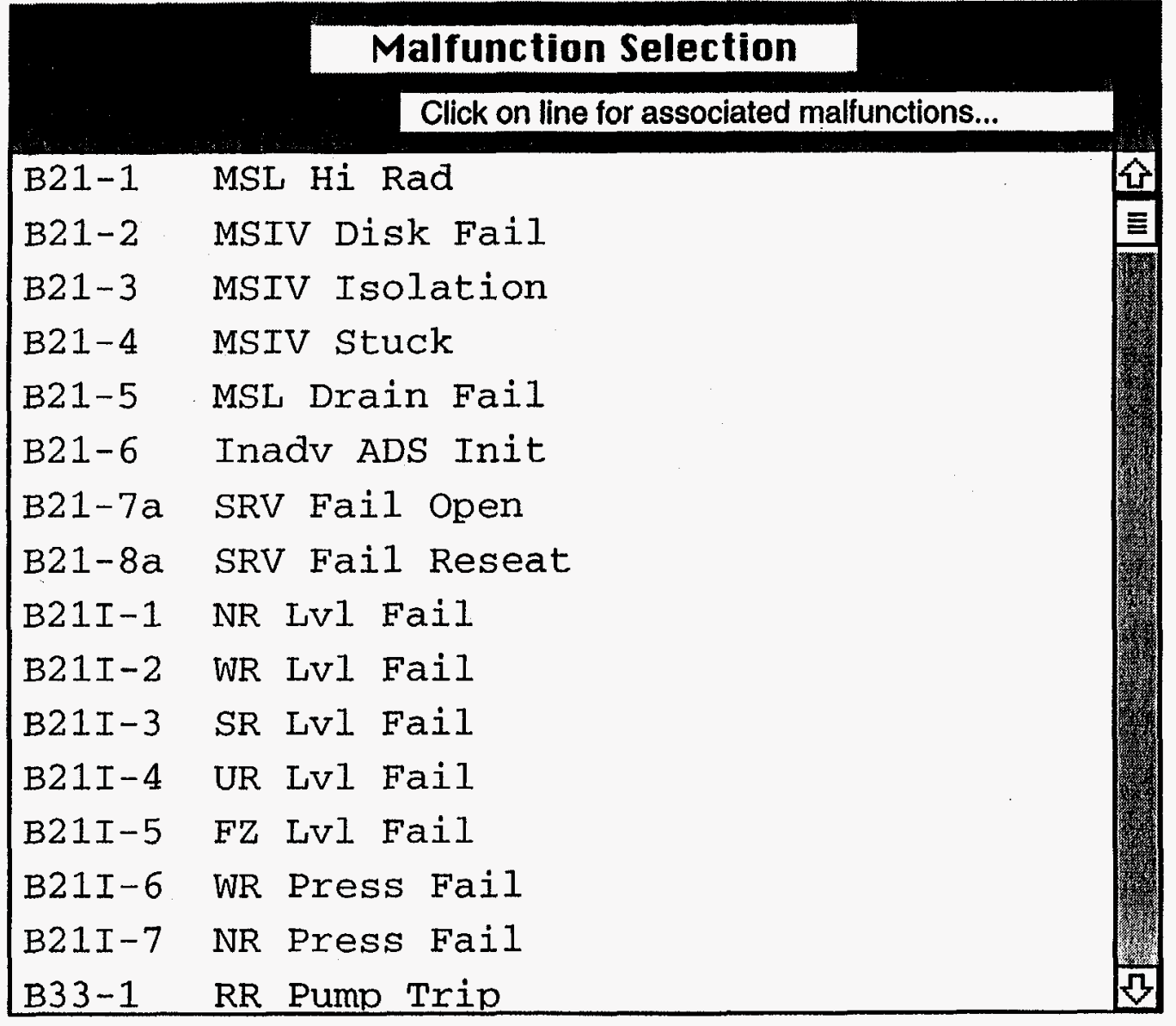




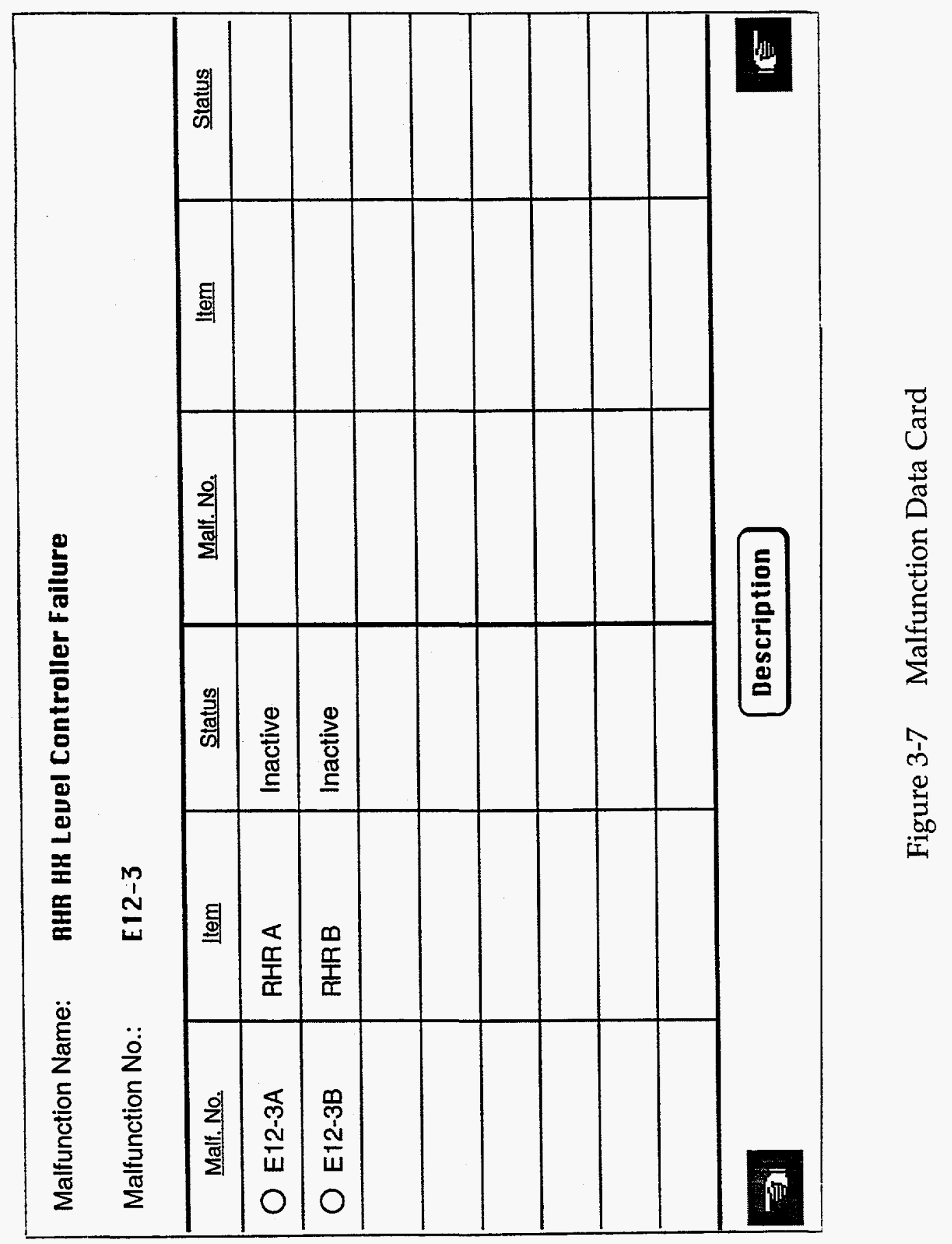




\section{Malfunction Name: $\quad$ RHR Pump Trip \\ Malfunction No.: $\quad$ E12-1 \\ Type: Discrete \\ Options: $\quad$ A-Pump A $\quad$ B - Pump B $\quad$ C - Pump C $\quad$ D - All Pumps}

\section{Cause: $\quad$ overcurrent trip of pump}

This malfunction causes the RHR pump to trip, opening of the pump motor breaker, and actuation of the RHR pump motor auto trip annunciator. RHR pump flow decreases to $0 \mathrm{gpm}$ (as per pump coastdown) and pump motor current abruptly decreases to 0 amps.

If the affected RHR pump was being used to increase reactor vessel water level, then water level will increase at a slower rate or stop increasing, depending on the operating status of other injection equipment.

If the affected RHR pump was being used for shutdown cooling, then reactor coolant temperature will decrease at a slower rate or start increasing, depending on the amount of decay heat and the status of other heat removal equipment.

Removal of this malfunction will allow the restart of the affected RHR Pump(s). If an auto initiation signal is present, the pump will automatically restart. 


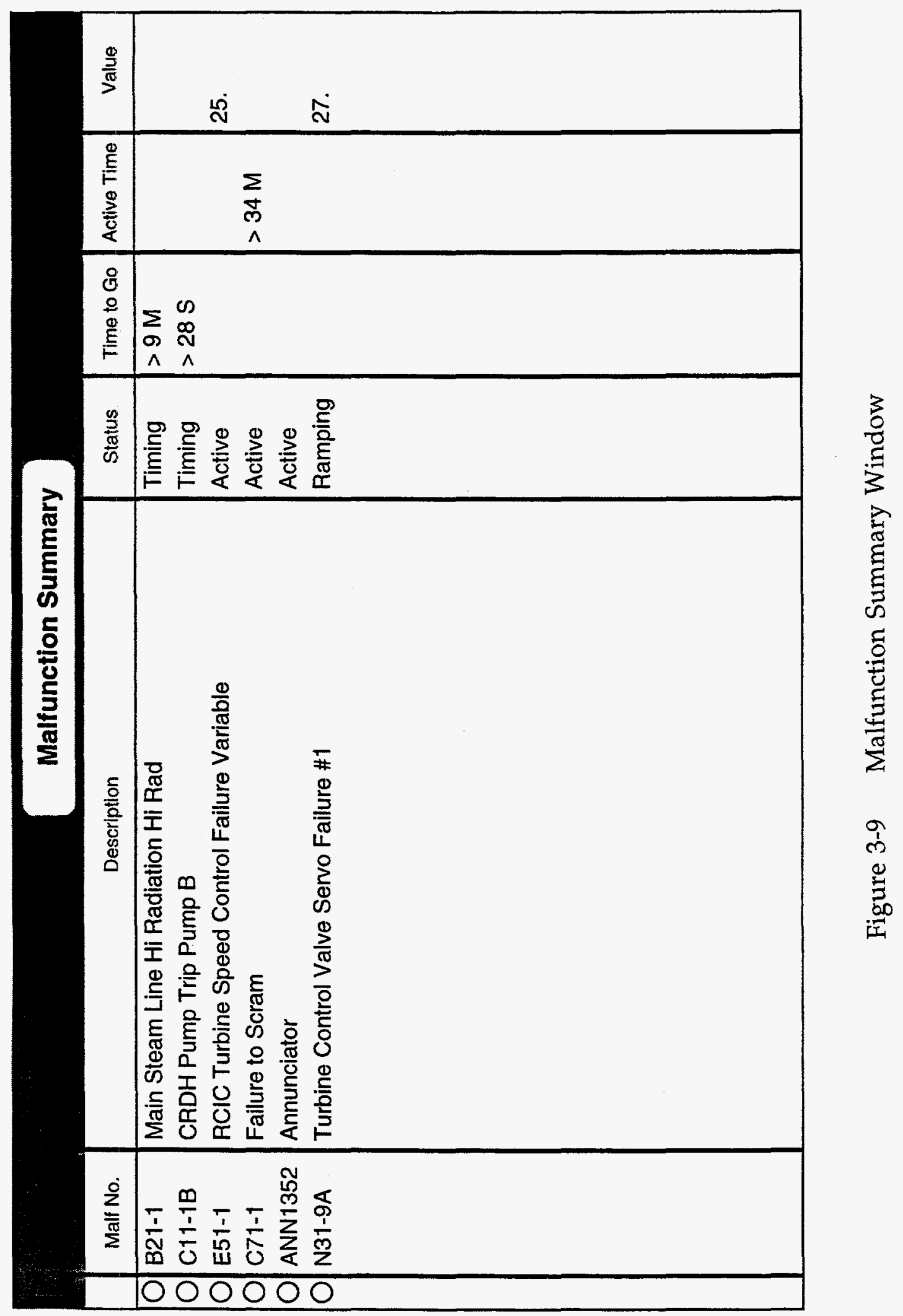




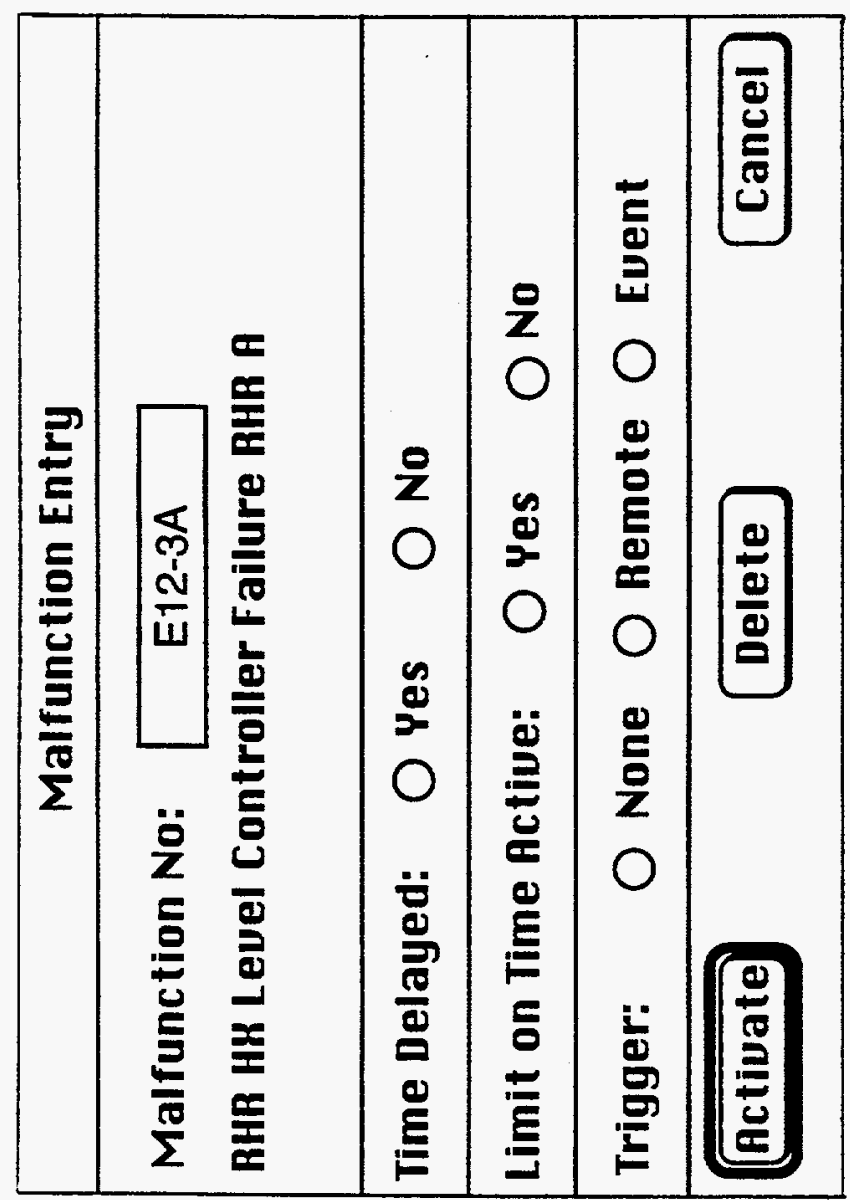

吾 


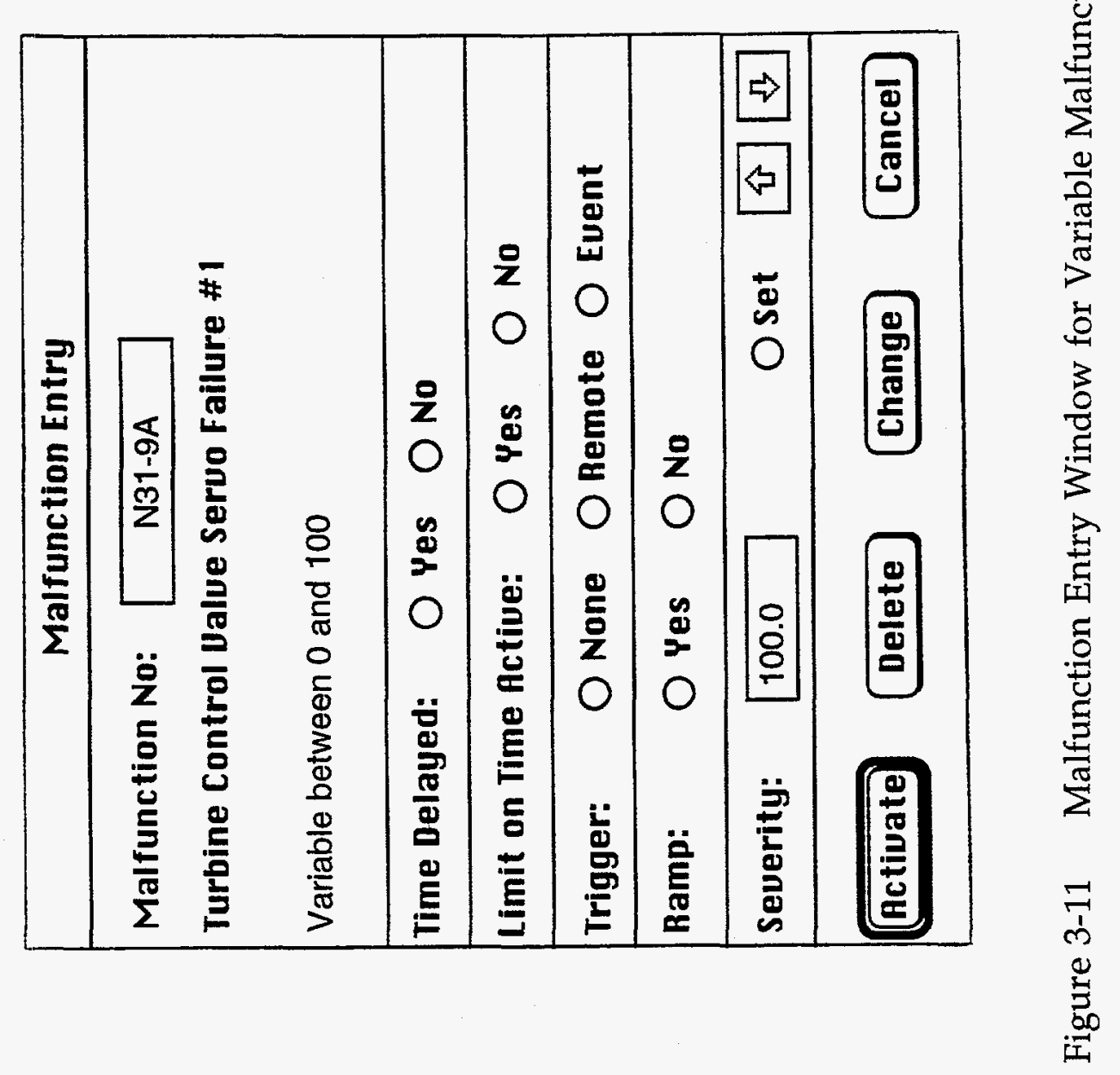




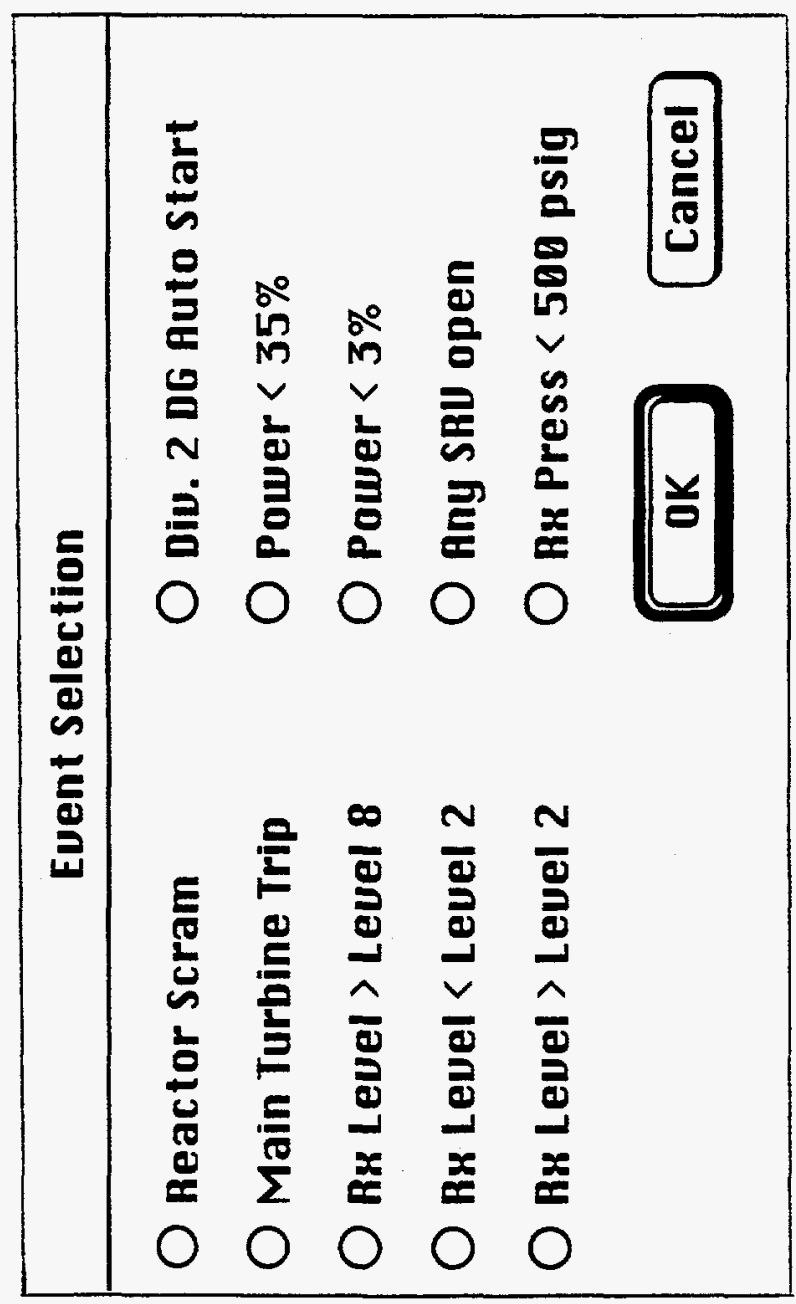

范

咭 


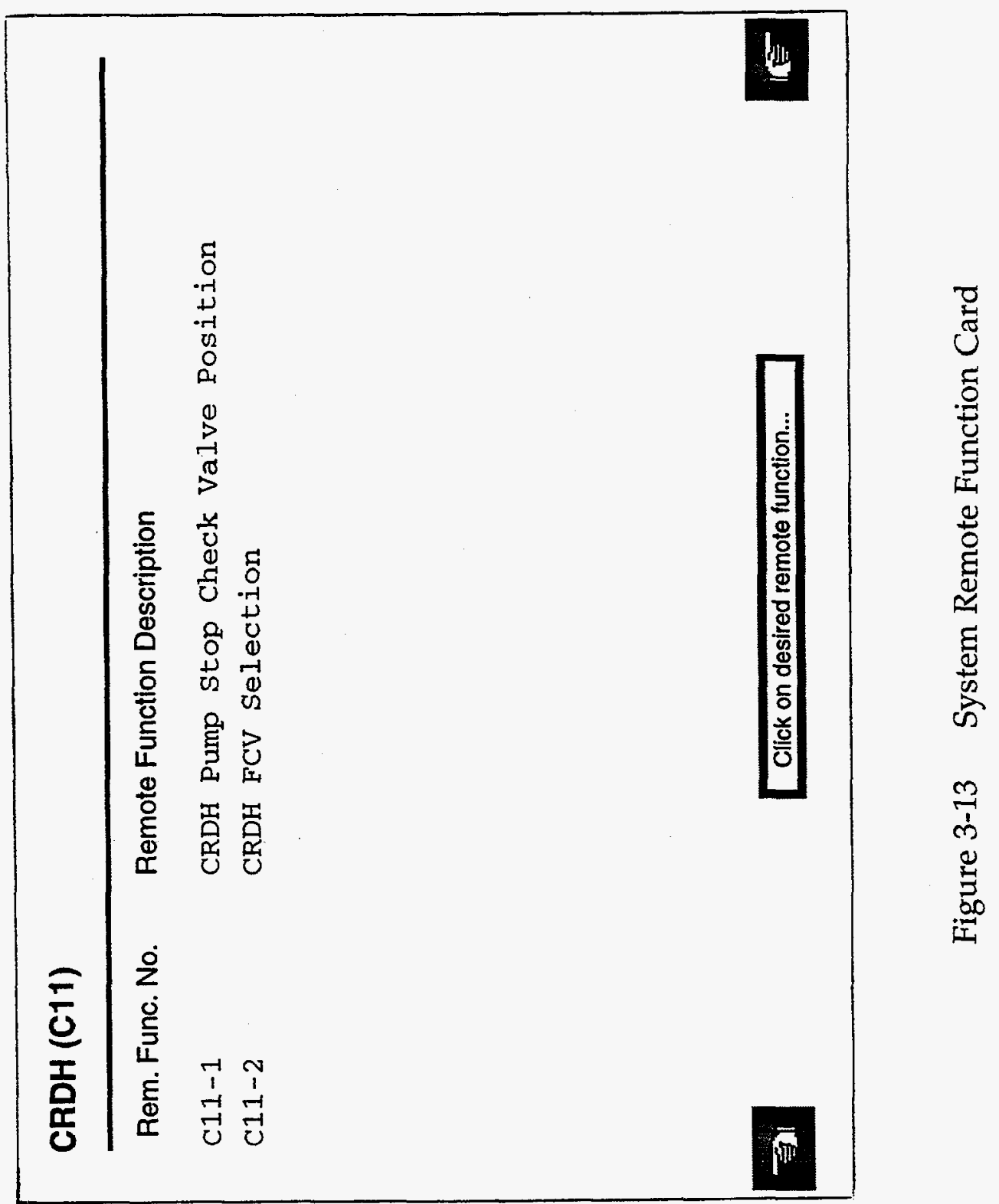




\begin{tabular}{|ll|} 
& \multicolumn{1}{c|}{$\begin{array}{c}\text { Remote Function Selection } \\
\text { Click on line for associated remote functions... }\end{array}$} \\
\hline B13-1 & BOP Isolation Bypass \\
B21-1 & MSIV LOW Level Iso1 \\
B21-2a & SRV Solenoid Fuses \\
B21-3 & SRV Low-Low Set Bypass \\
B21I-1 & PAMS Recorder Speed \\
B33-1 & RR Pump LOR Reset \\
B33-2 & EOC-RPT Bypass \\
C11-1 & CRDH Stop-Check Valve \\
C11-2 & CRDH FCV Select \\
C11R-1a & ROd Position Bypass \\
C11R-2 & Gang Rod Drive Speed \\
C11R-3 & LOw Power Setpoint Bypass \\
C34-1 & Setpoint Setdown Bypass \\
C34-2 & Setpoint Setdown Reset \\
C51-2 & APRM Calibration Gain \\
C51-3 & APRM Reference Reading
\end{tabular}

Figure 3-14 Remote Function Selection Window 


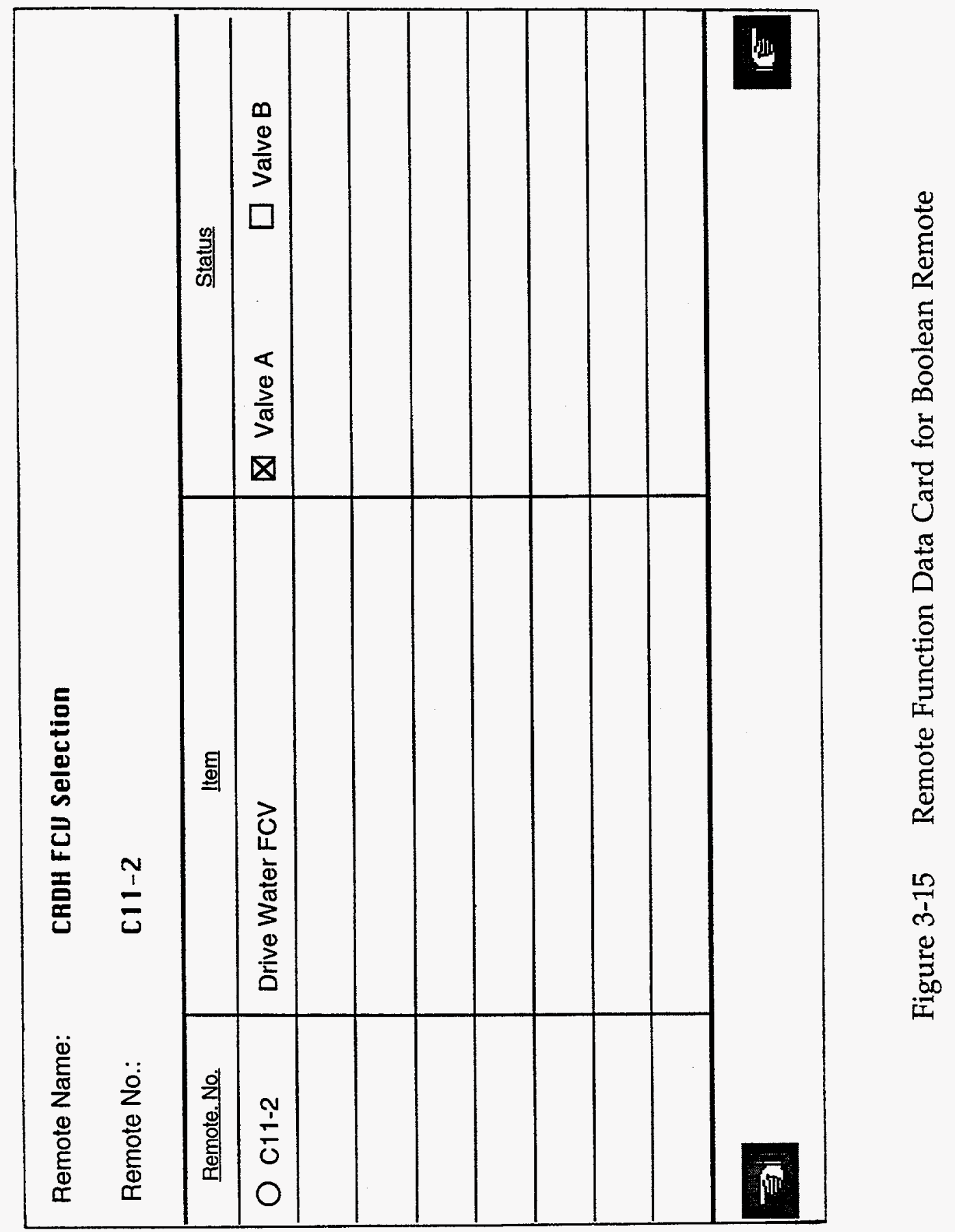




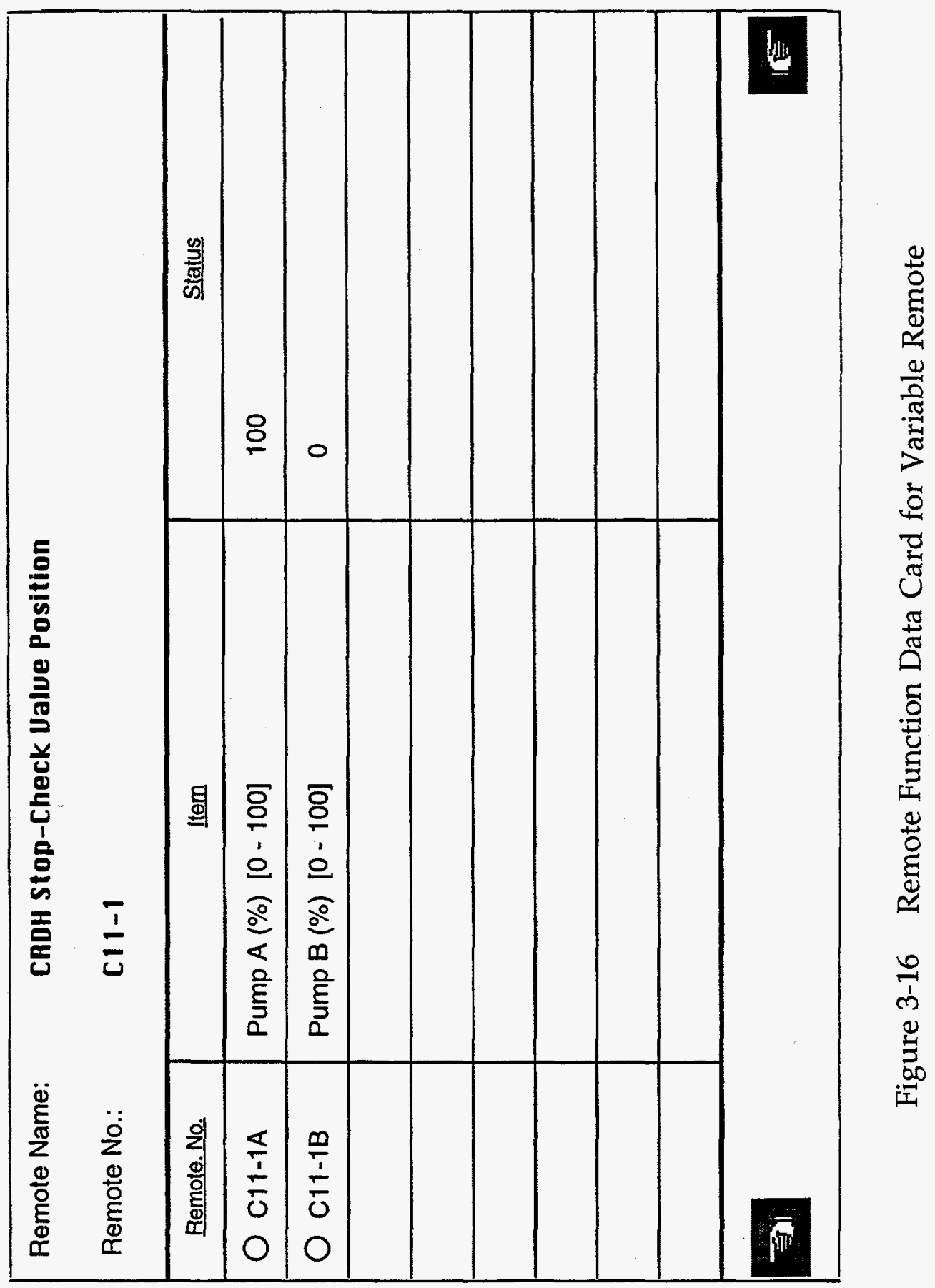




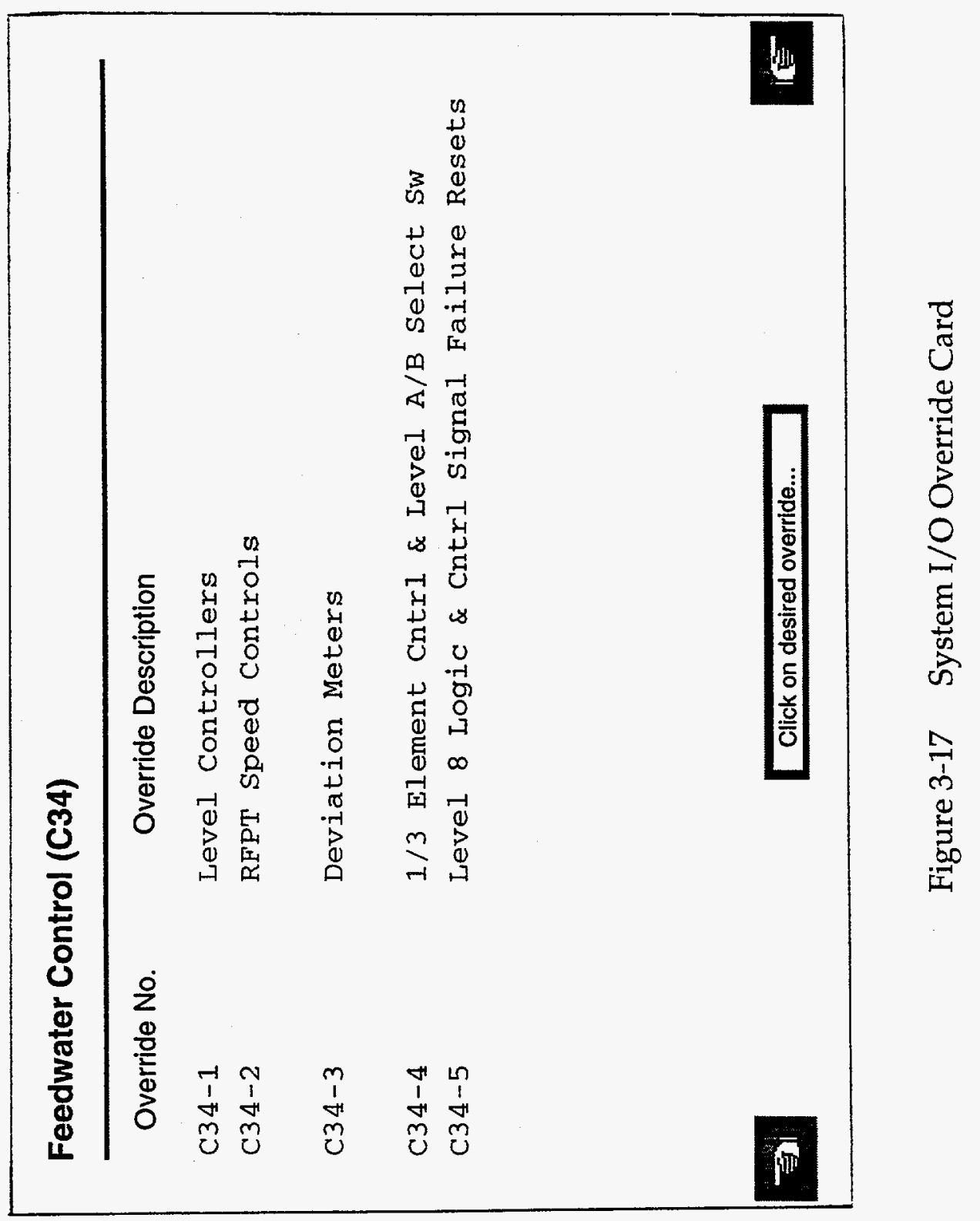




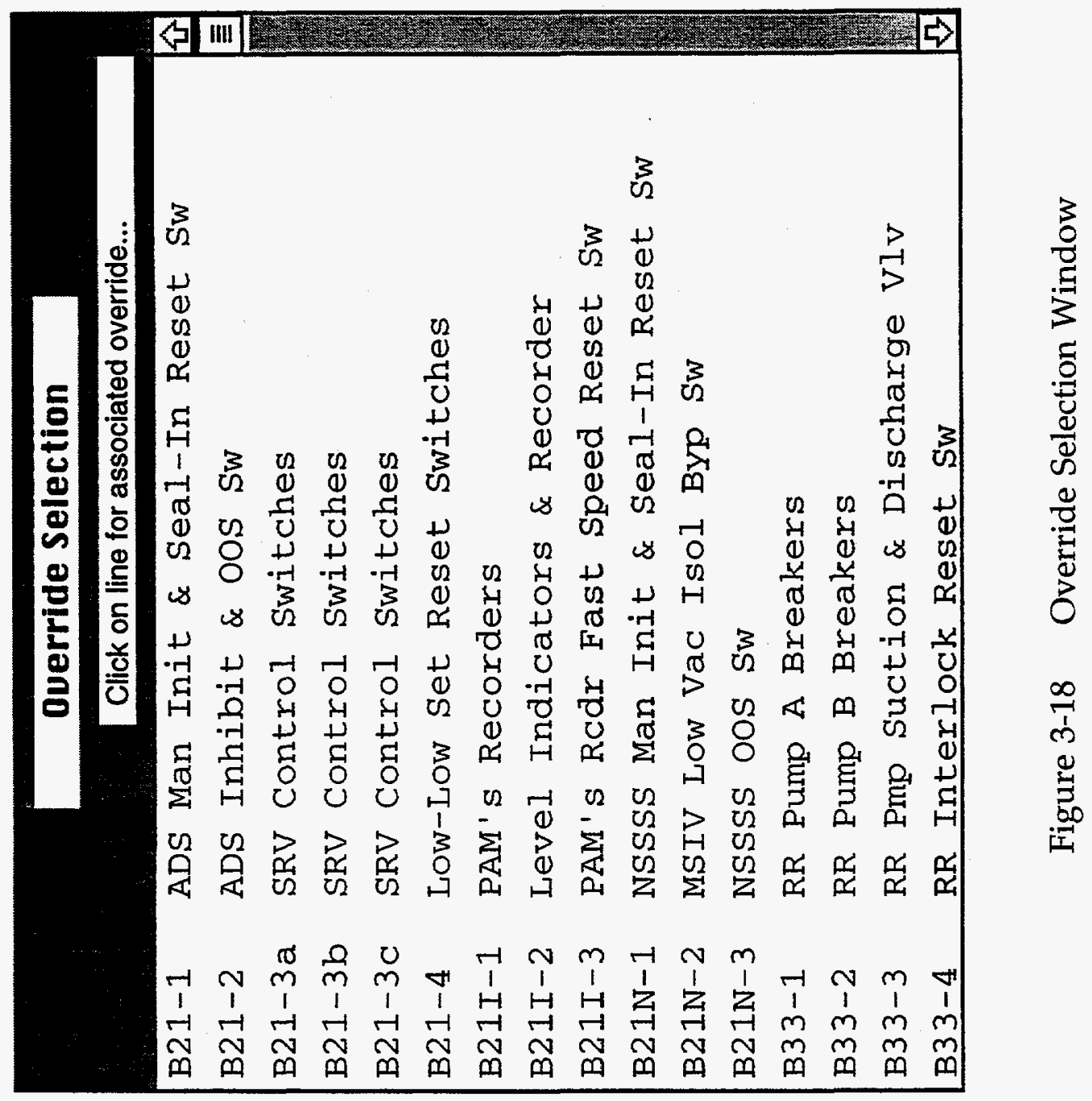




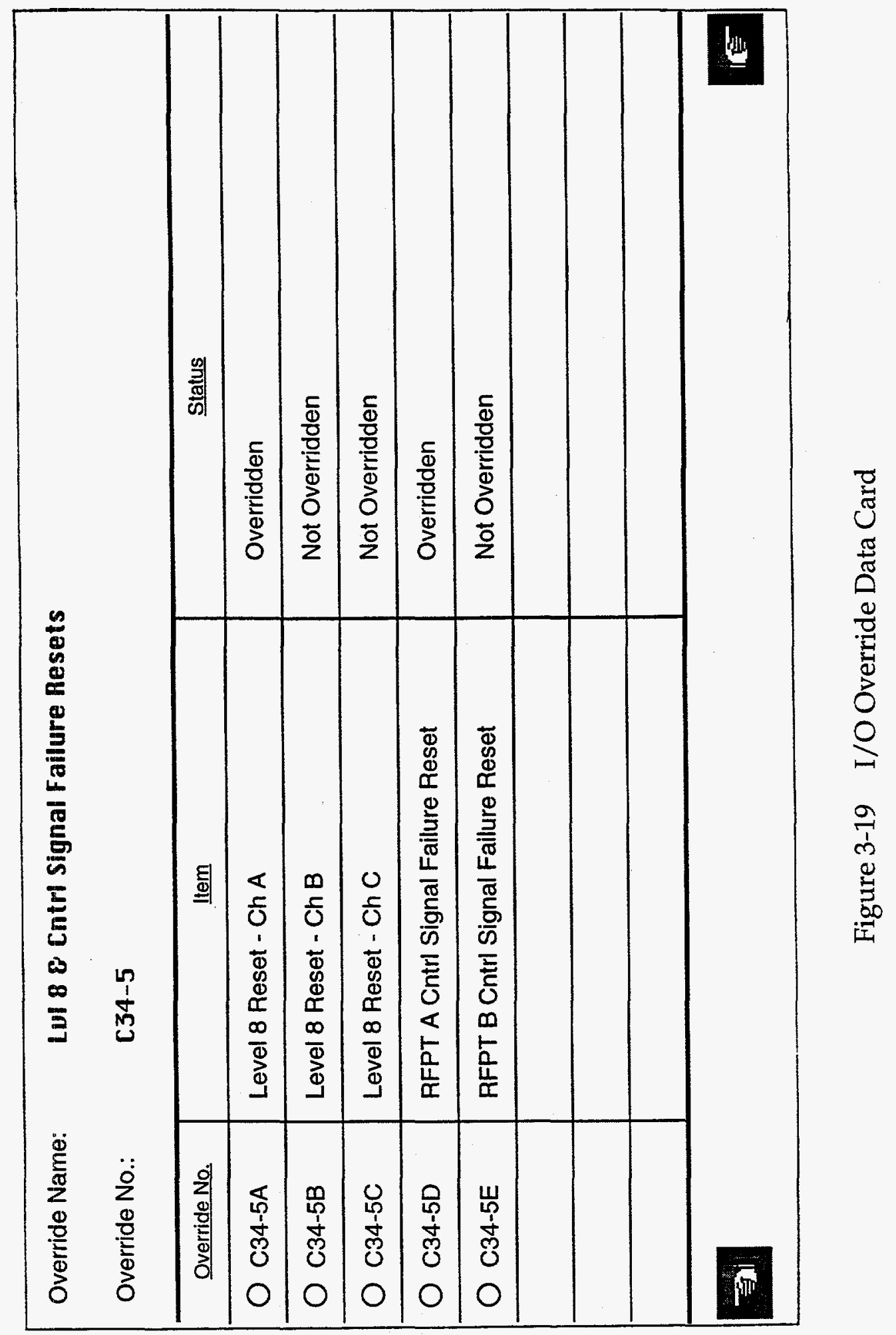




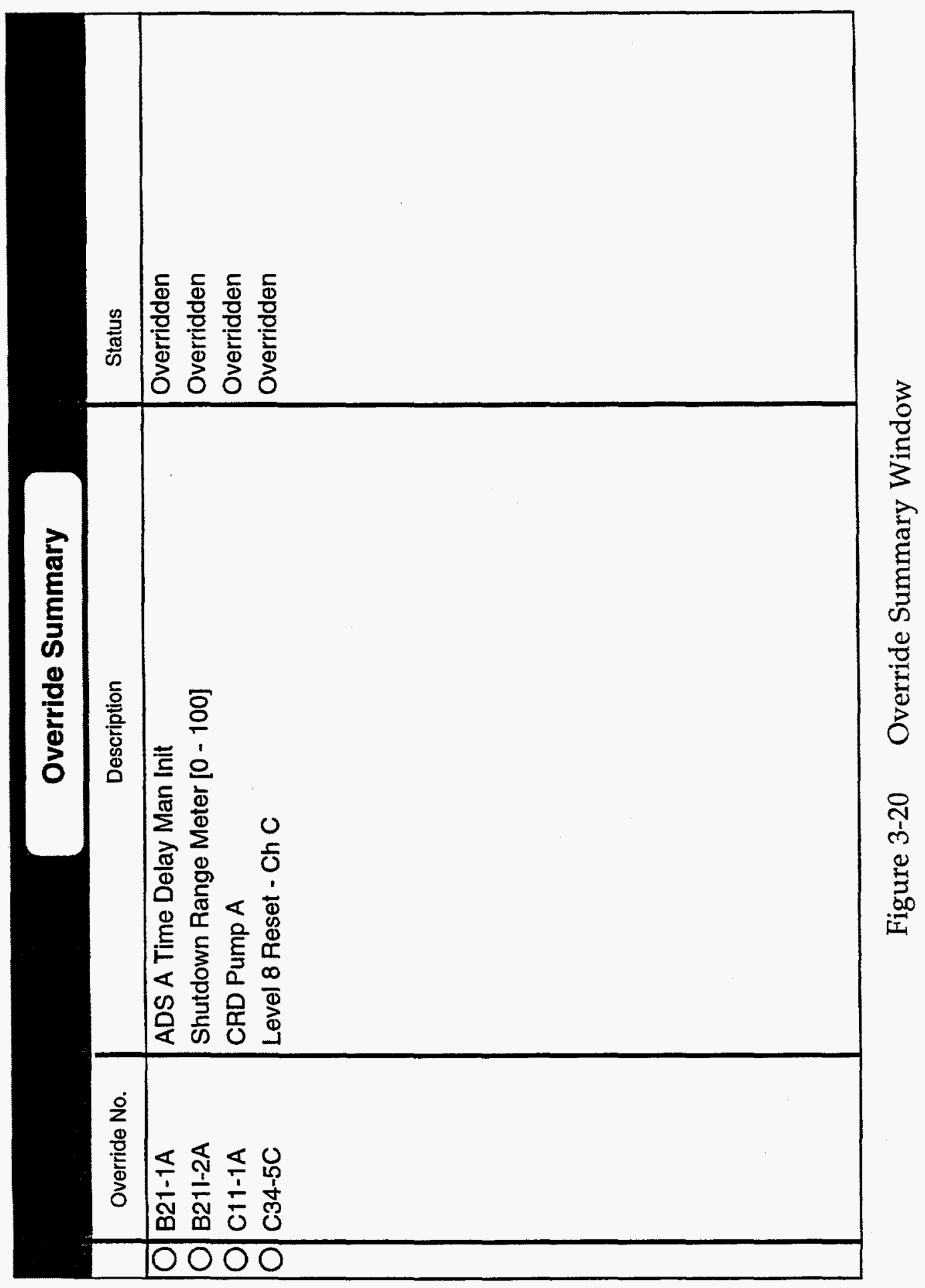




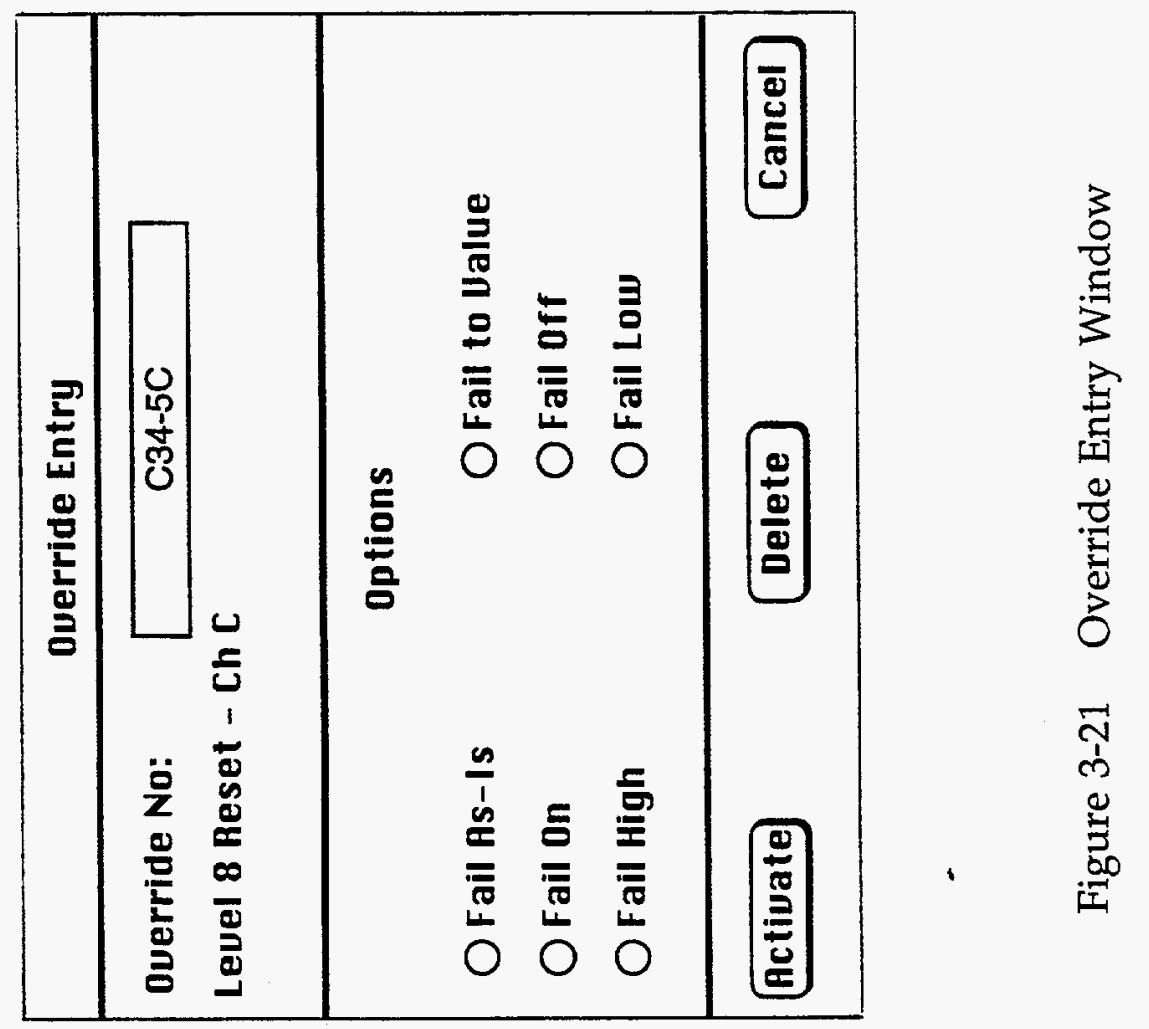




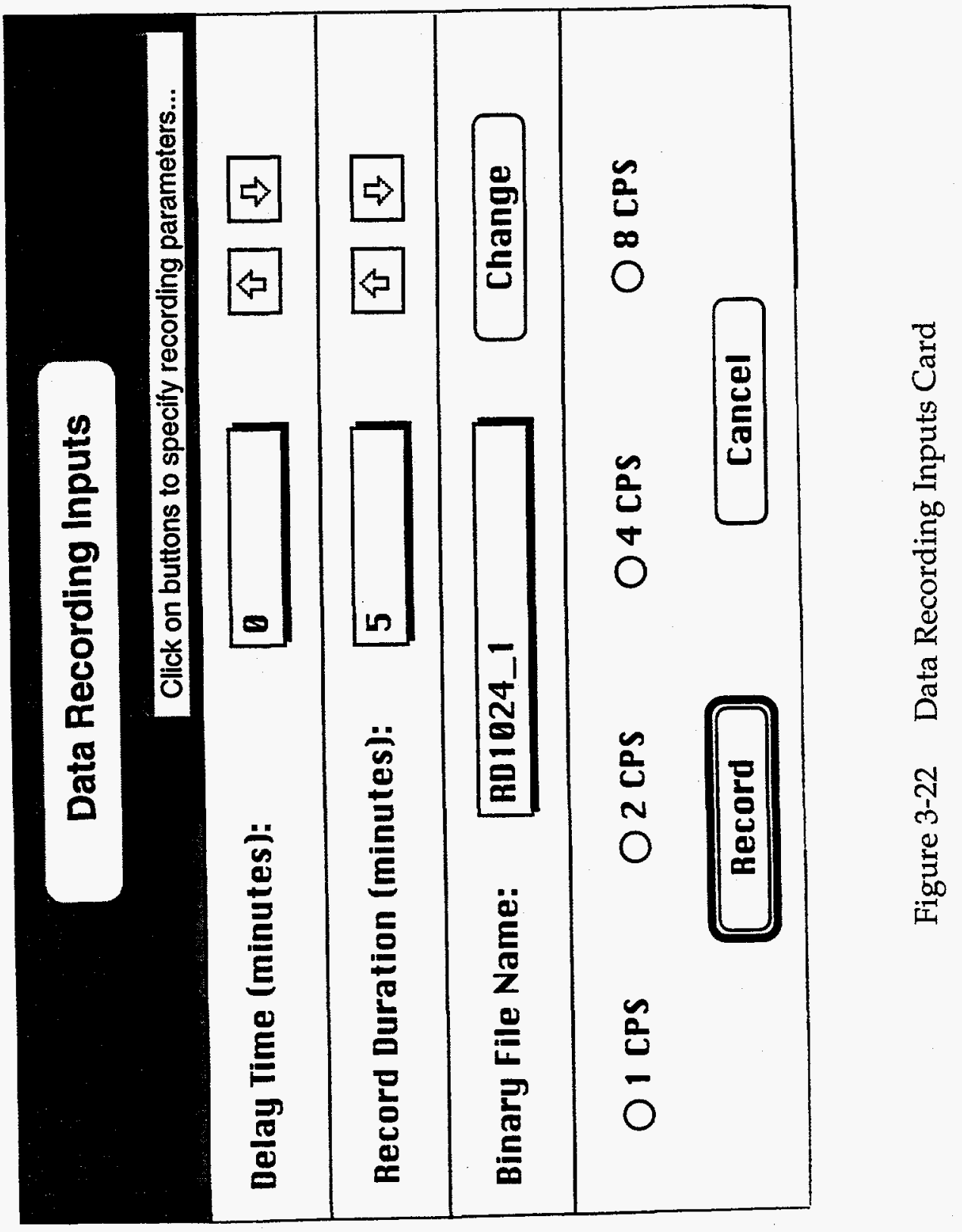




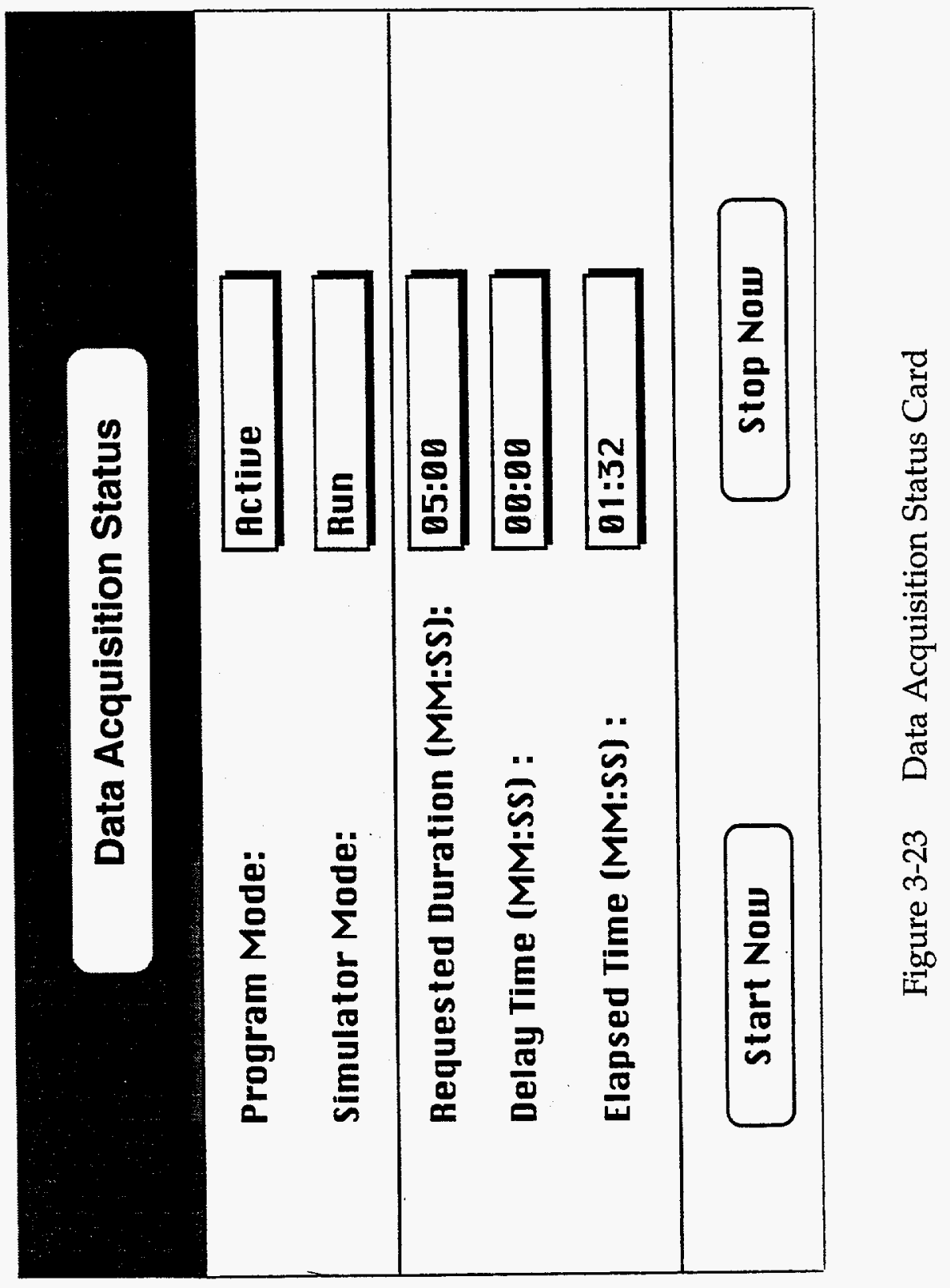




\begin{tabular}{|c|}
\hline Data Acquisition is complete. \\
\hline $\begin{array}{c}\text { Do you wish to convert and } \\
\text { transfer the collected data to } \\
\text { an Excel file now? }\end{array}$ \\
Yes \\
\hline
\end{tabular}




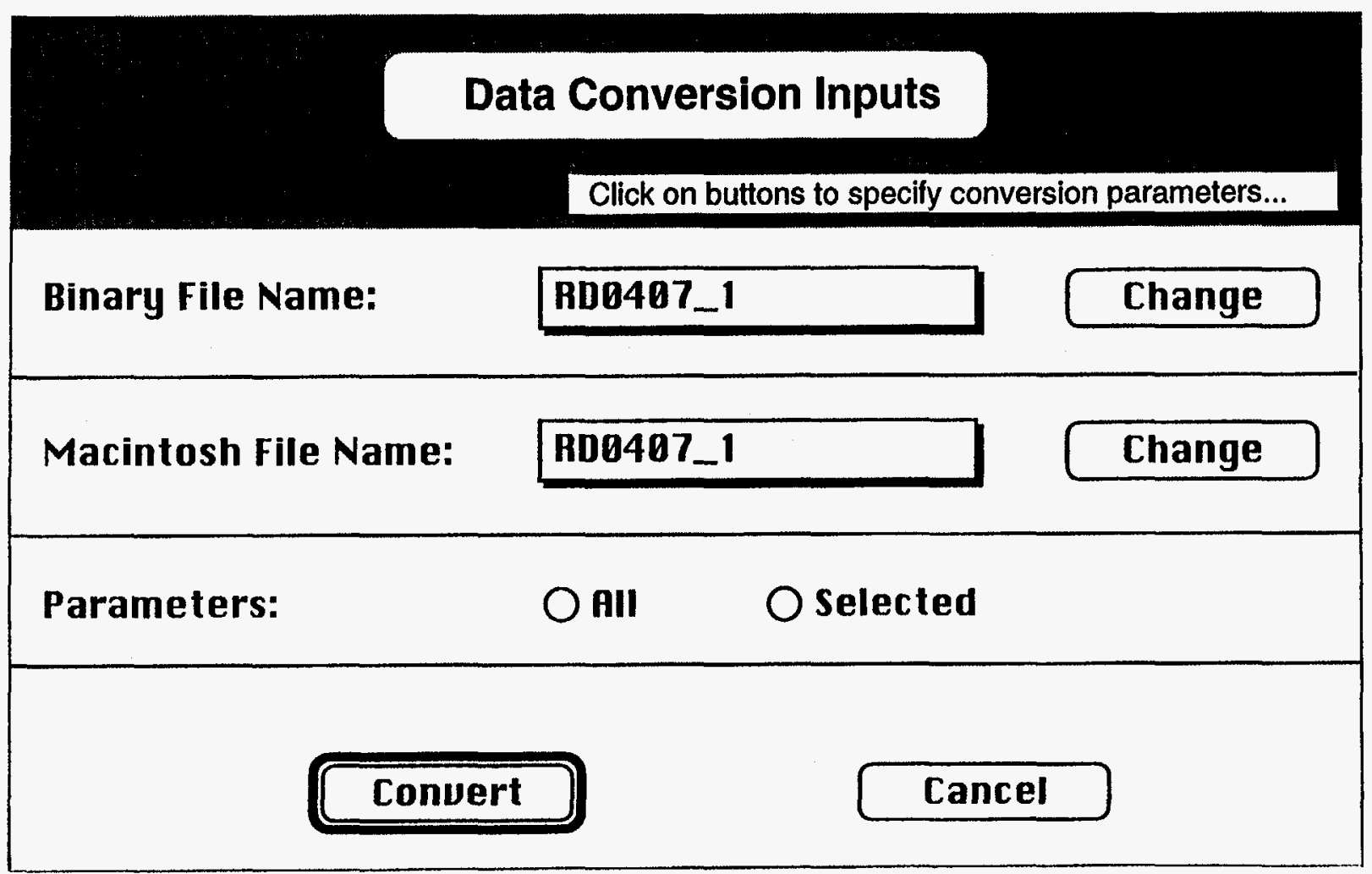

Figure 3-25 Data Conversion Inputs Card 


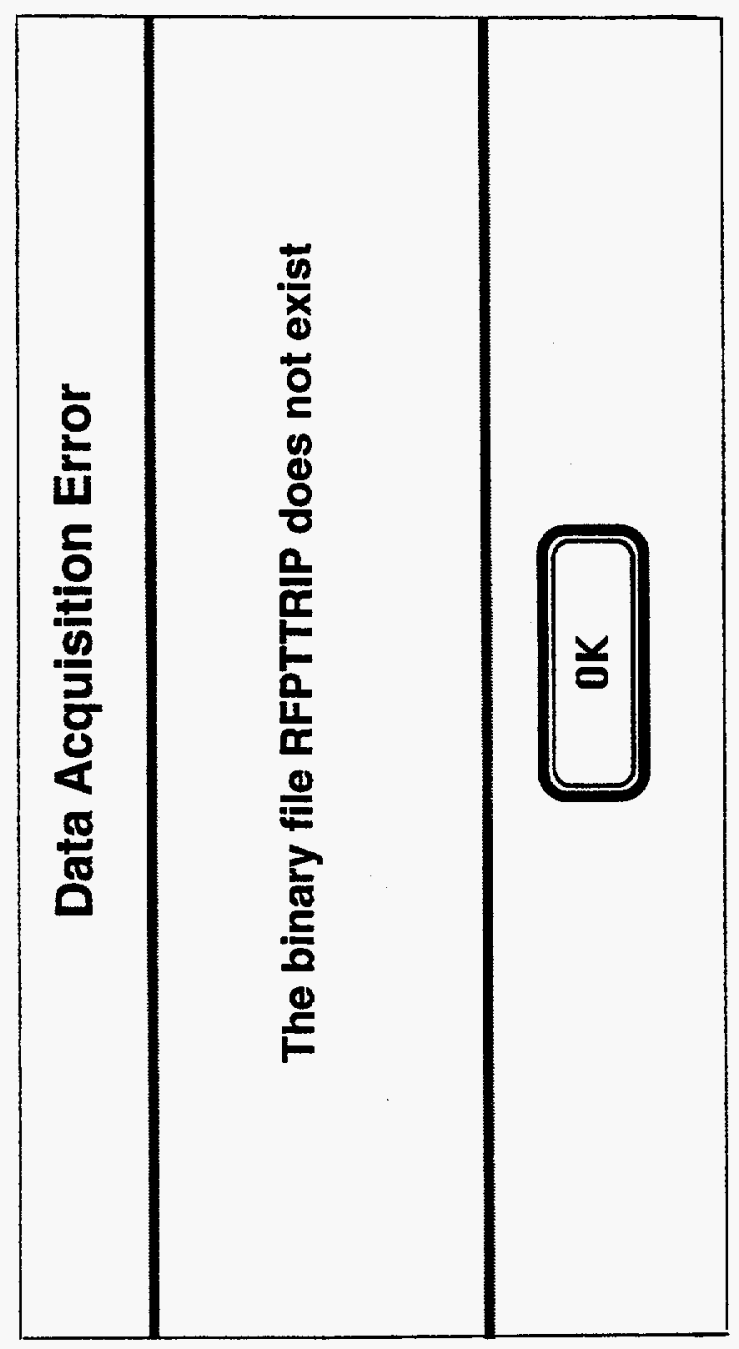



ஸै 


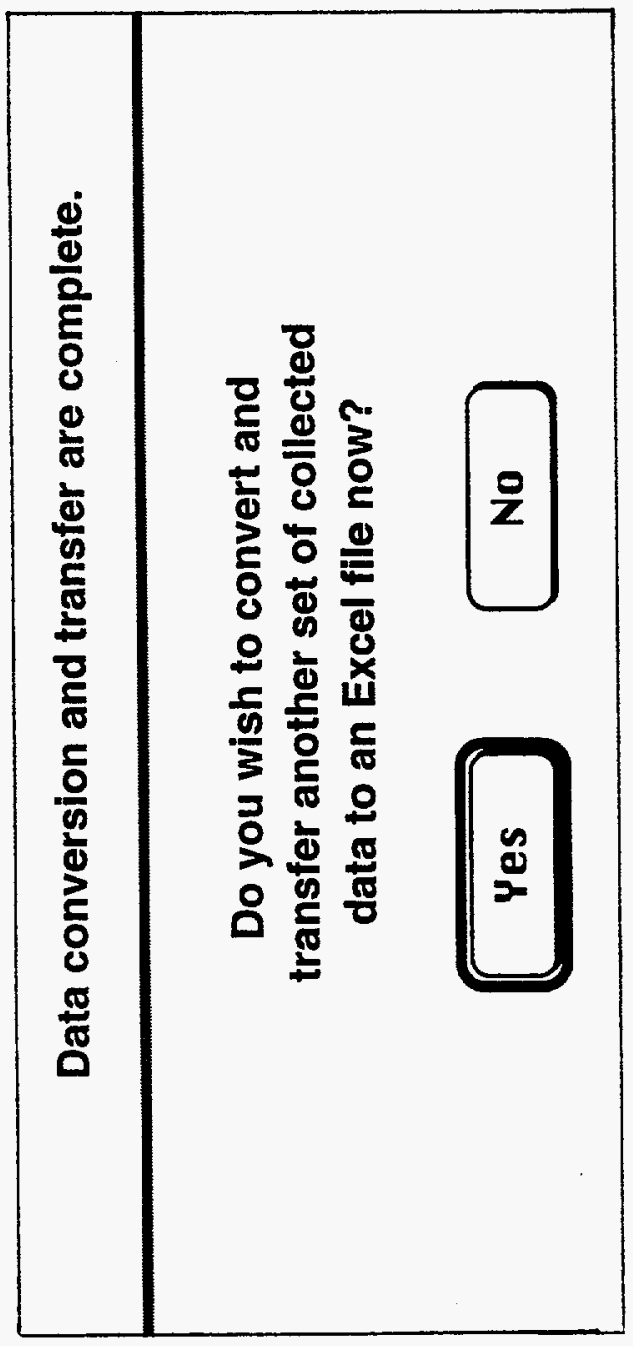

总

సิ 


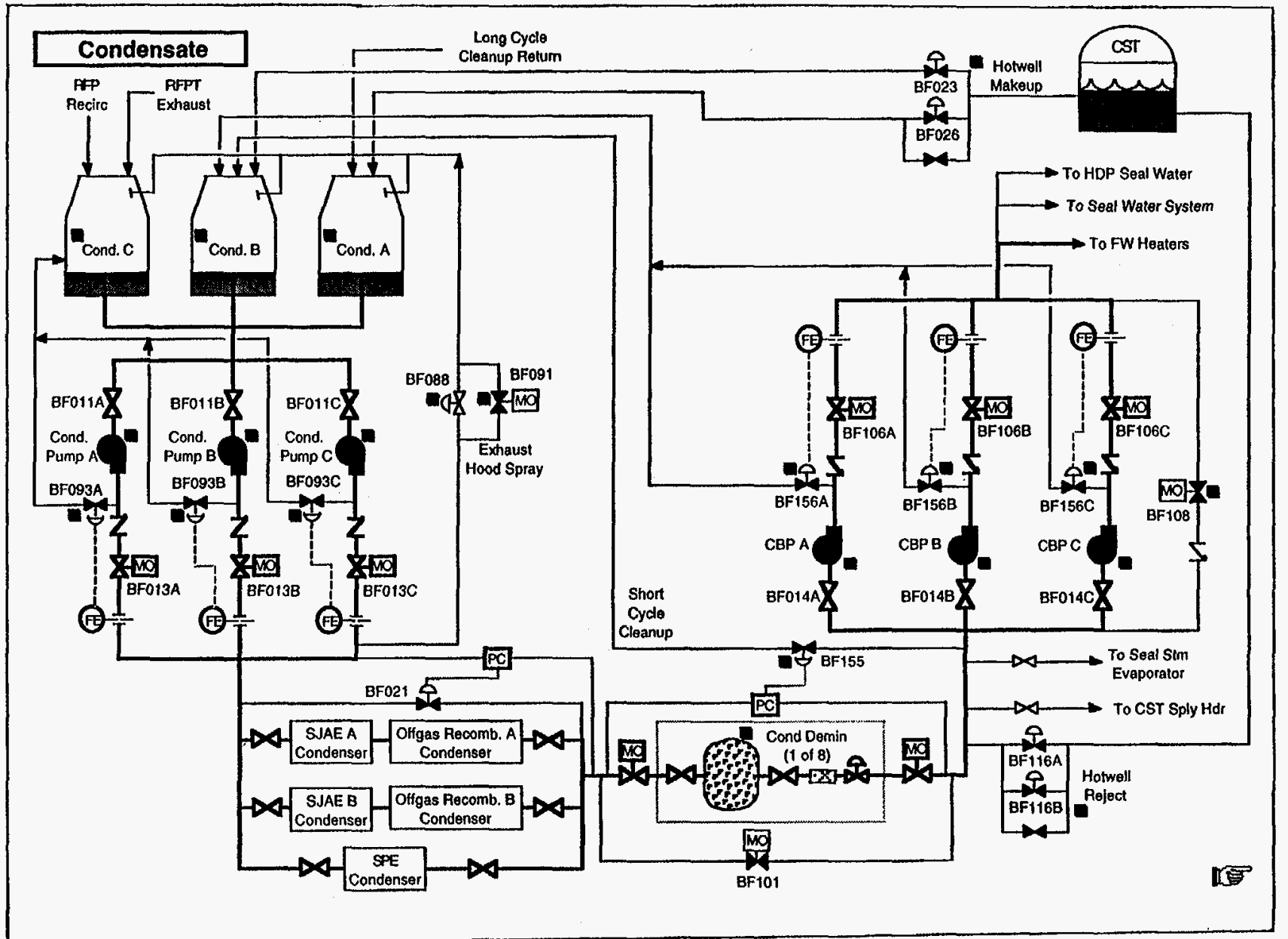

Figure 3-28 Interactive P\&ID 


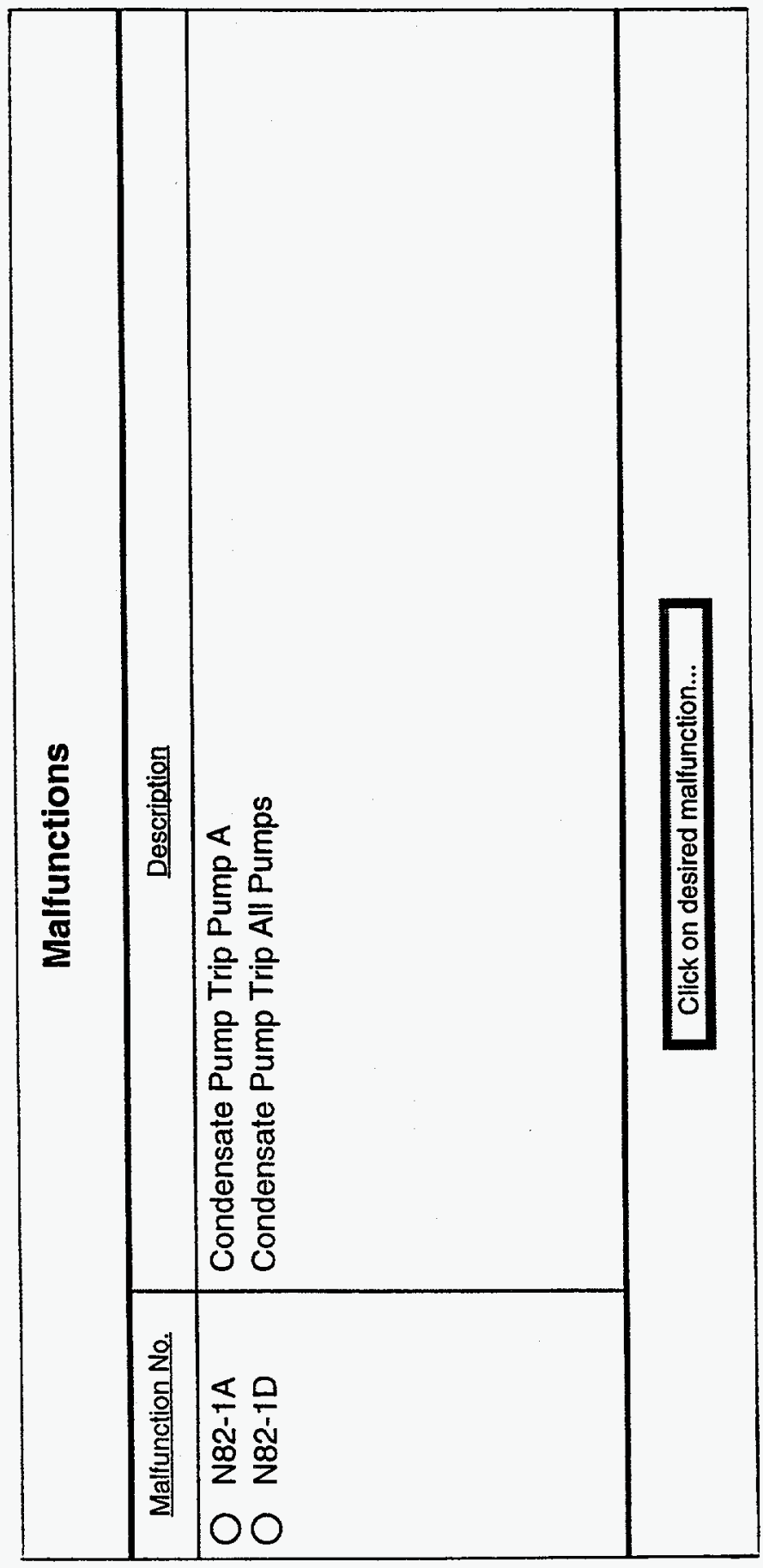

苟 


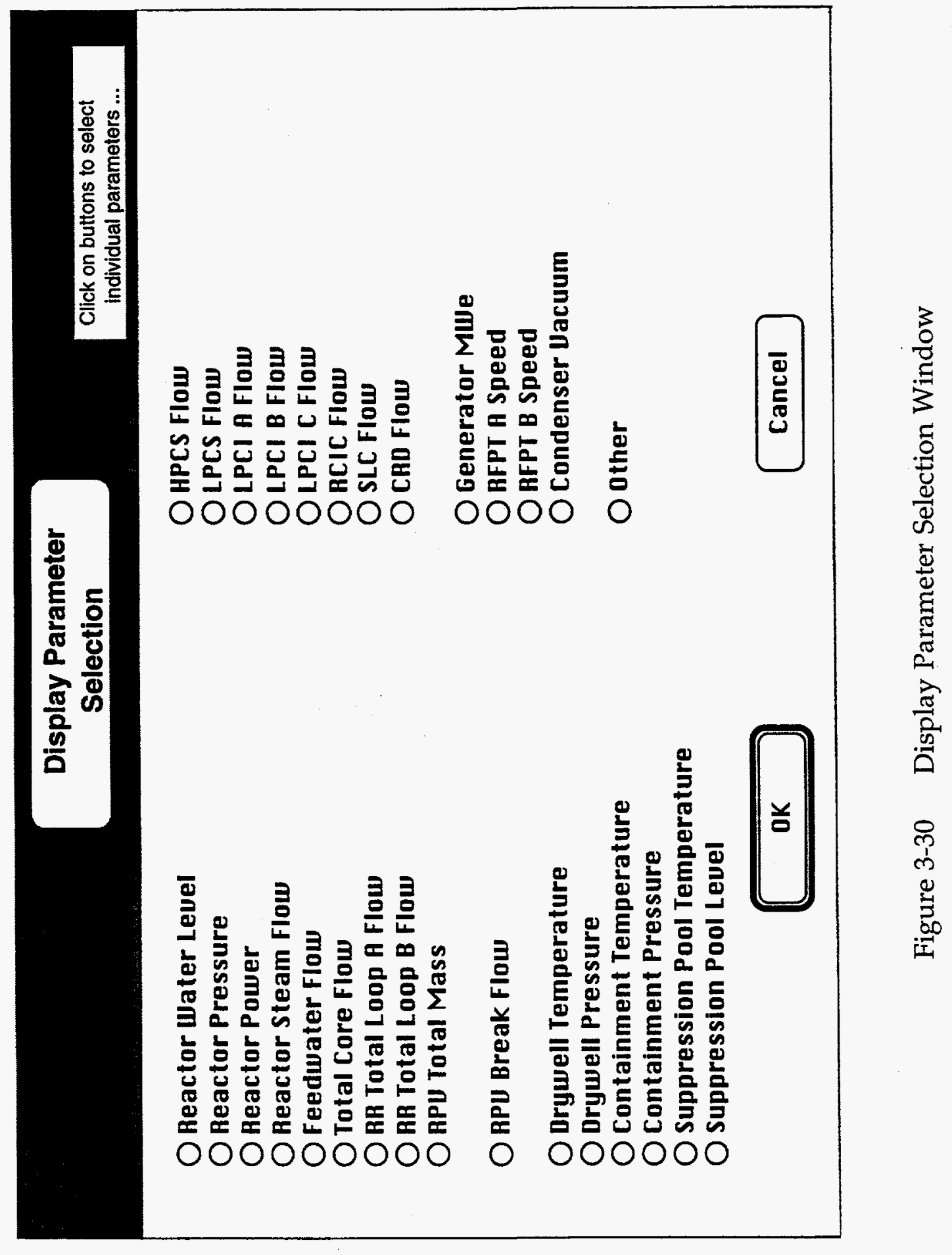




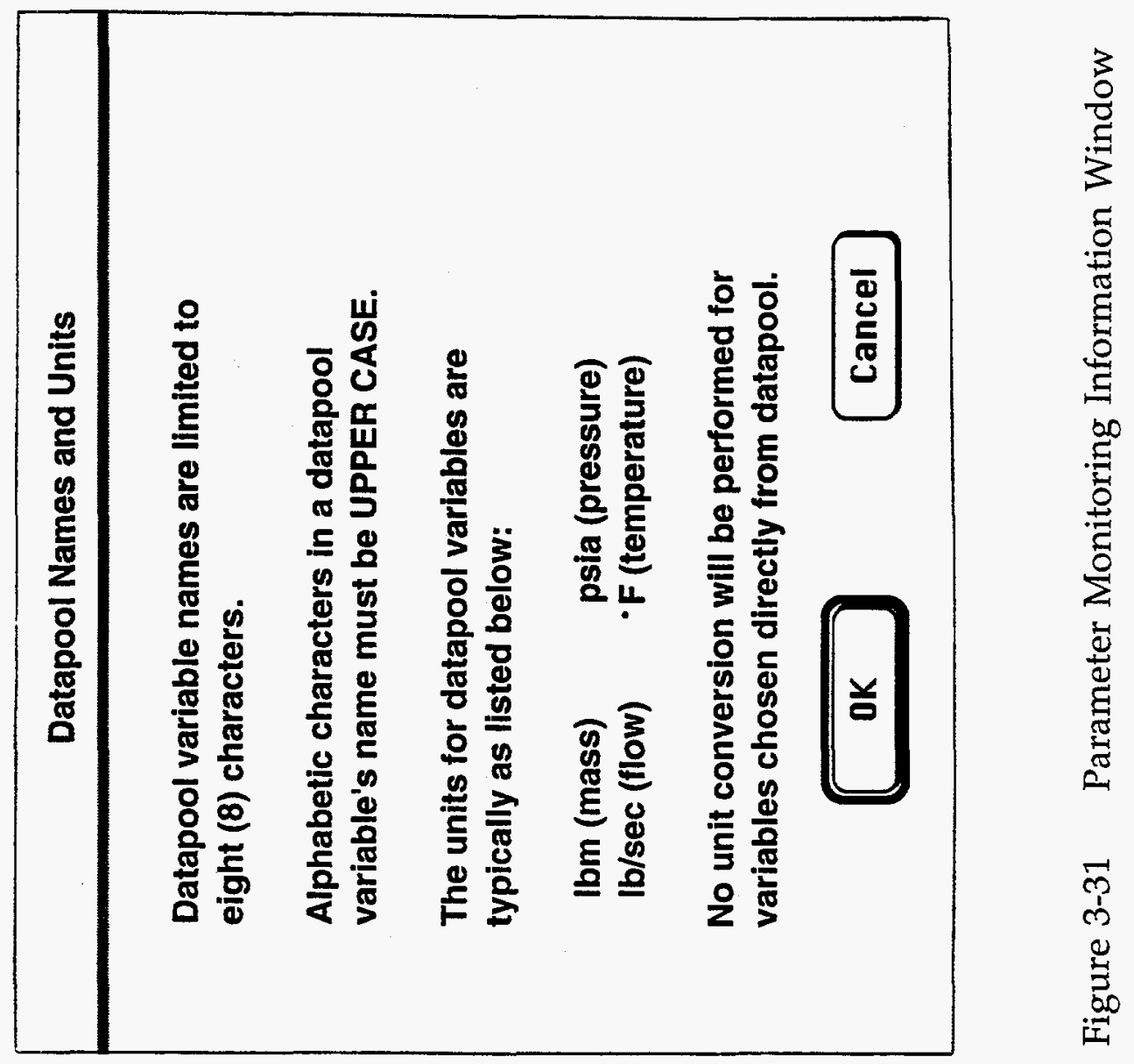




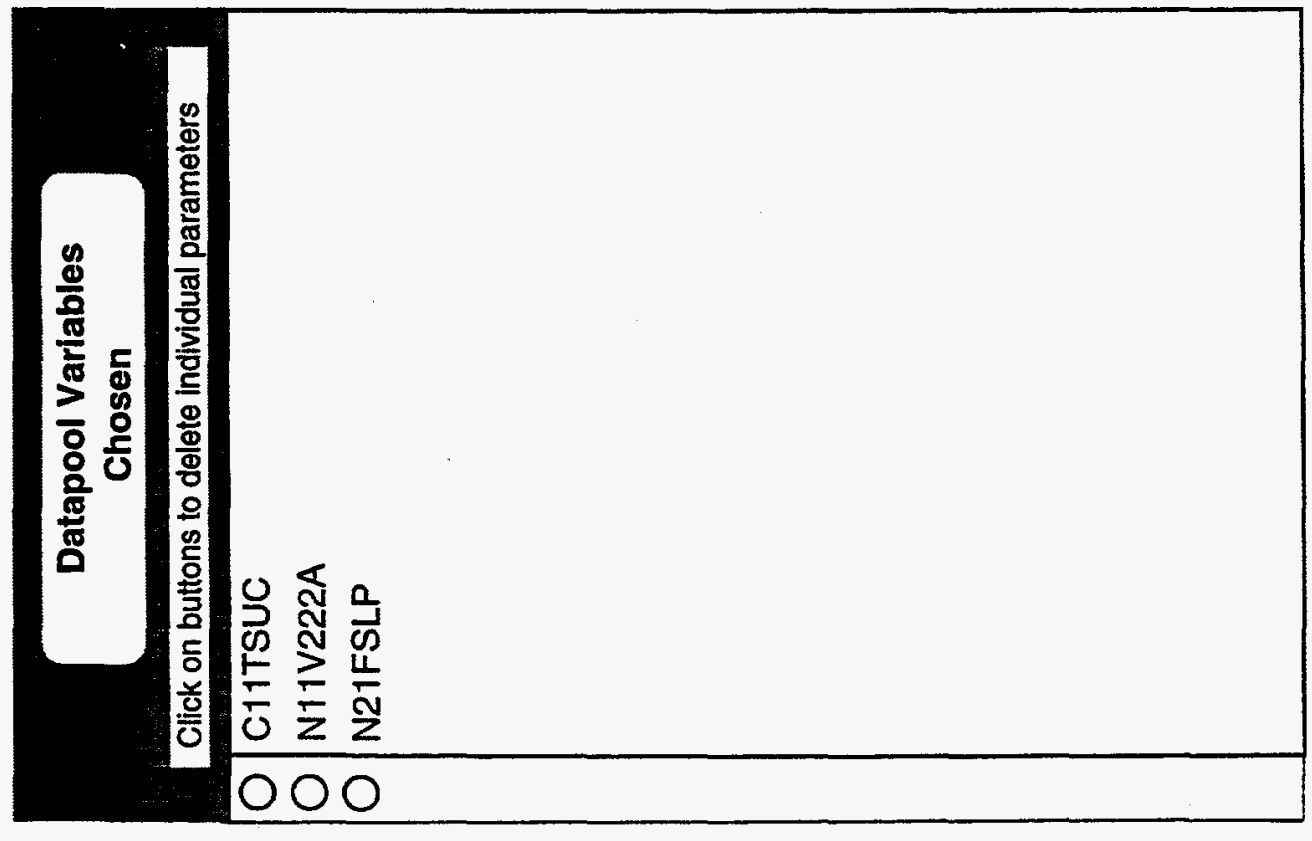

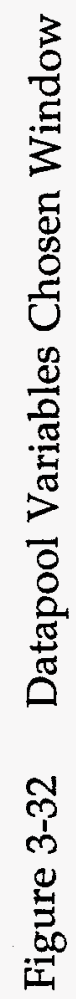




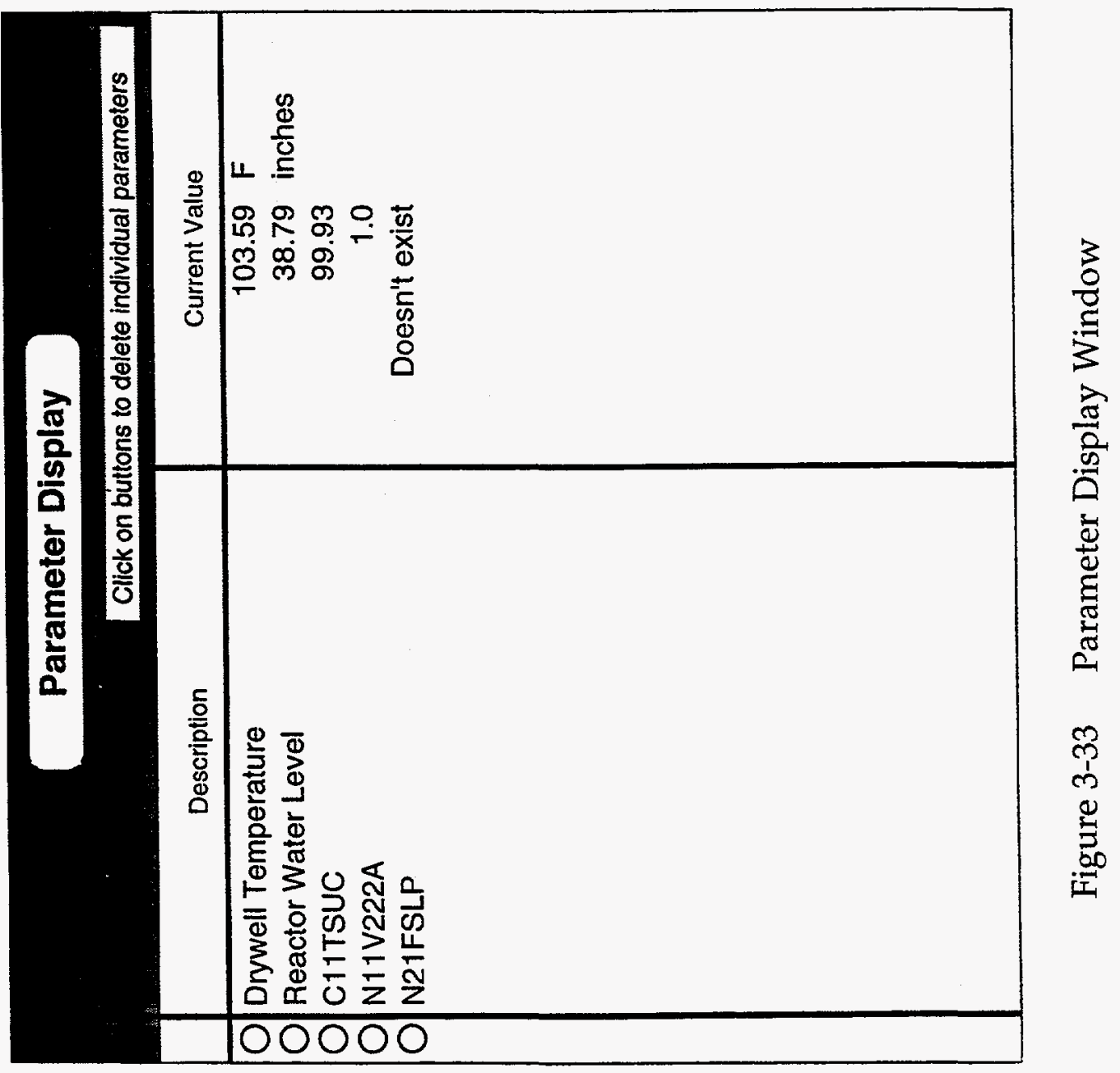




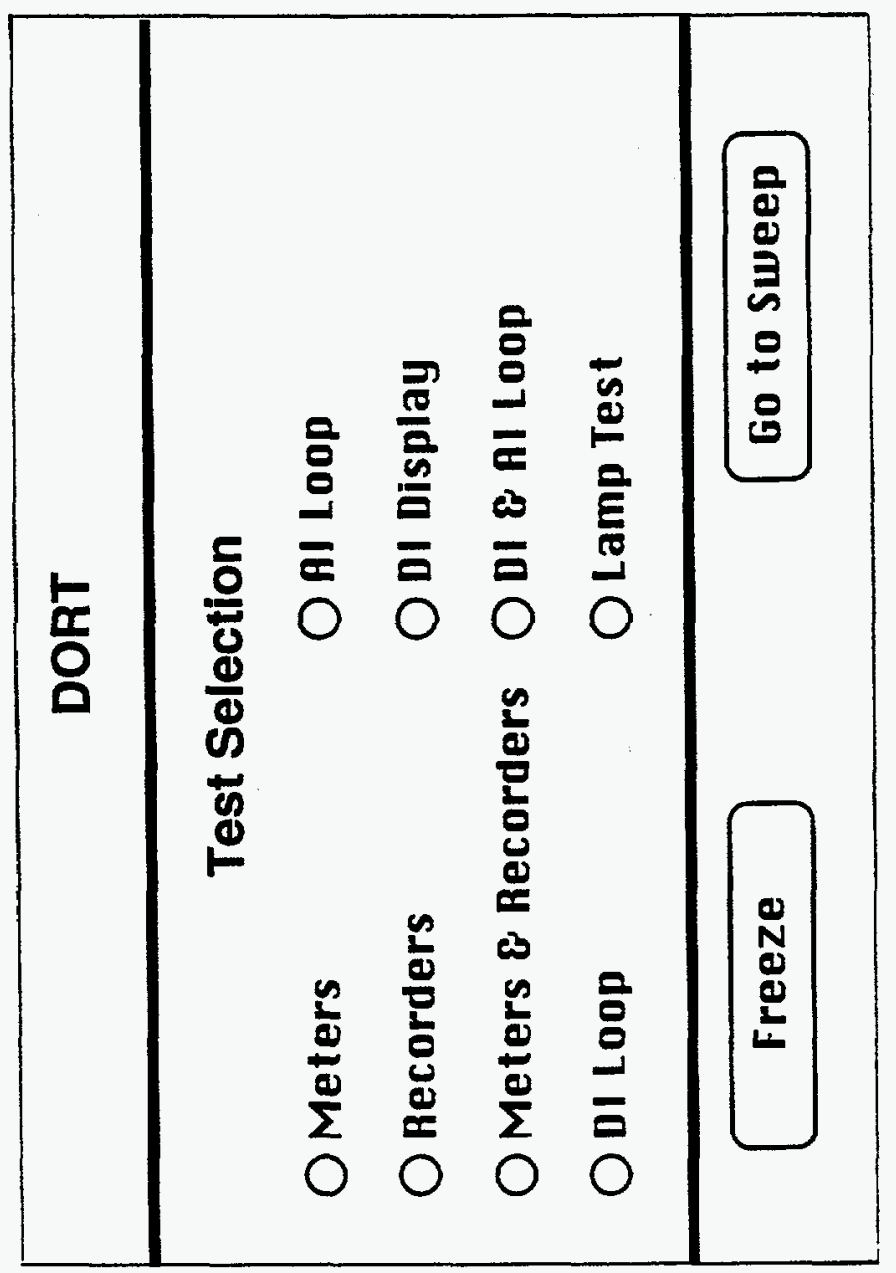

草

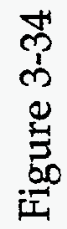




\section{MACINTOSH INSTRUCTOR STATION HARDWARE DESCRIPTION}

The Instructor Station is implemented using a Macintosh Quadra 700 computer (as a minimum). The computer is equipped with eight MBytes of memory, a 200 MByte hard disk, a built-in Ethernet card, a 21" Macintosh color monitor, 1 MByte of Video RAM, a keyboard, and a mouse.

The Macintosh communicates with the host computer via Ethernet using standard TCP/IP protocol. 


\section{ADDITIONAL IMPLEMENTATIONS}

As part of the purchase agreement for the CE Simulator, a major upgrade of the computer system, simulation software, and panel I/O was performed prior to its installation at the TTC. The Instructor Station provided with the CE Simulator had very few features and the user interface was cumbersome. Consequently, the original CE Instructor Station has also been replaced with the Macintosh Instructor Station. In this case, the host computer software tasks are implemented on a Unix-based workstation.

The computer system for the GE BWR/4 Simulator has recently been upgraded from two Encore 32/8780's to two Encore RSXTM Systems. Near-term plans for this simulator include replacement of the existing Instructor Station with the Macintosh Instructor Station. 


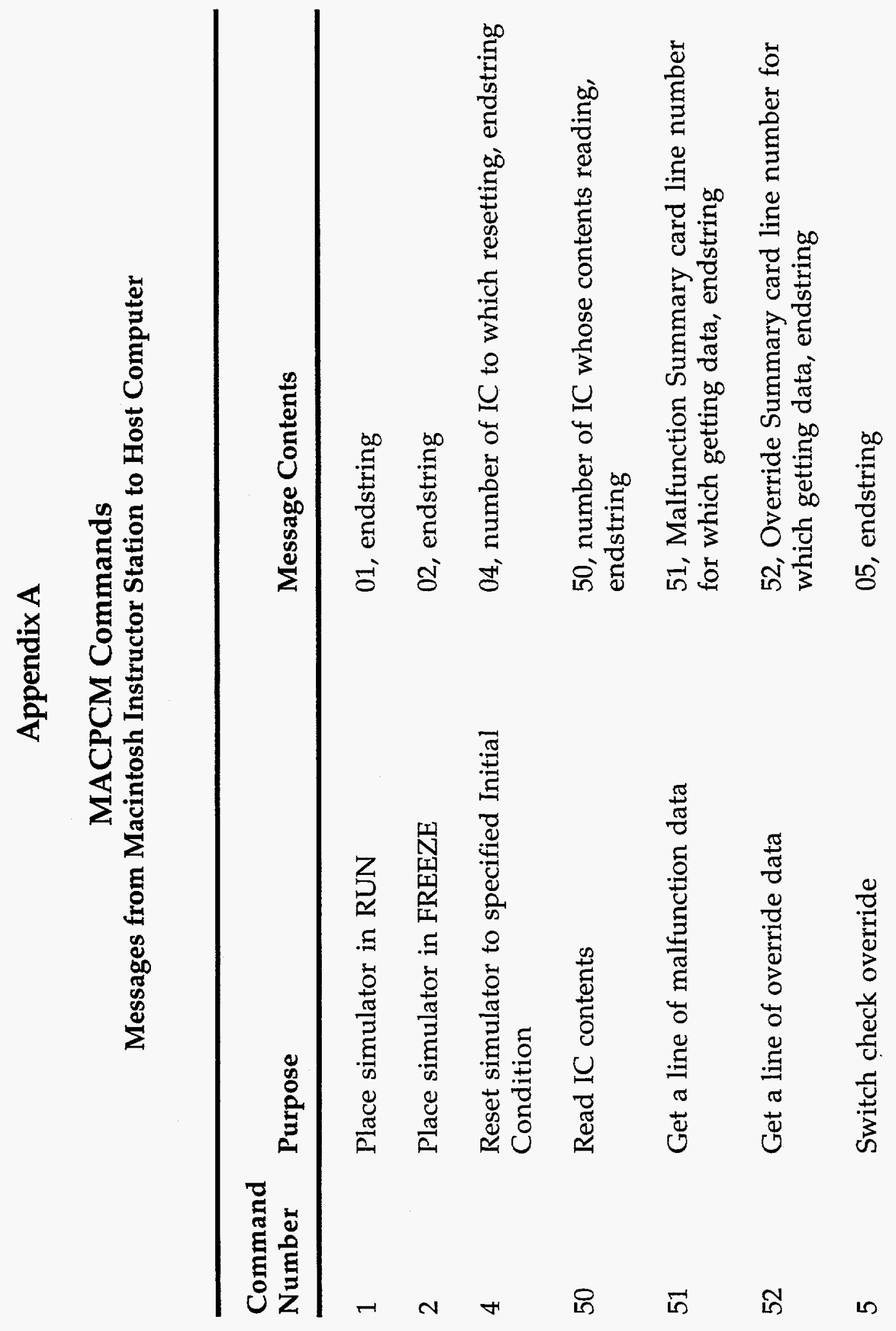




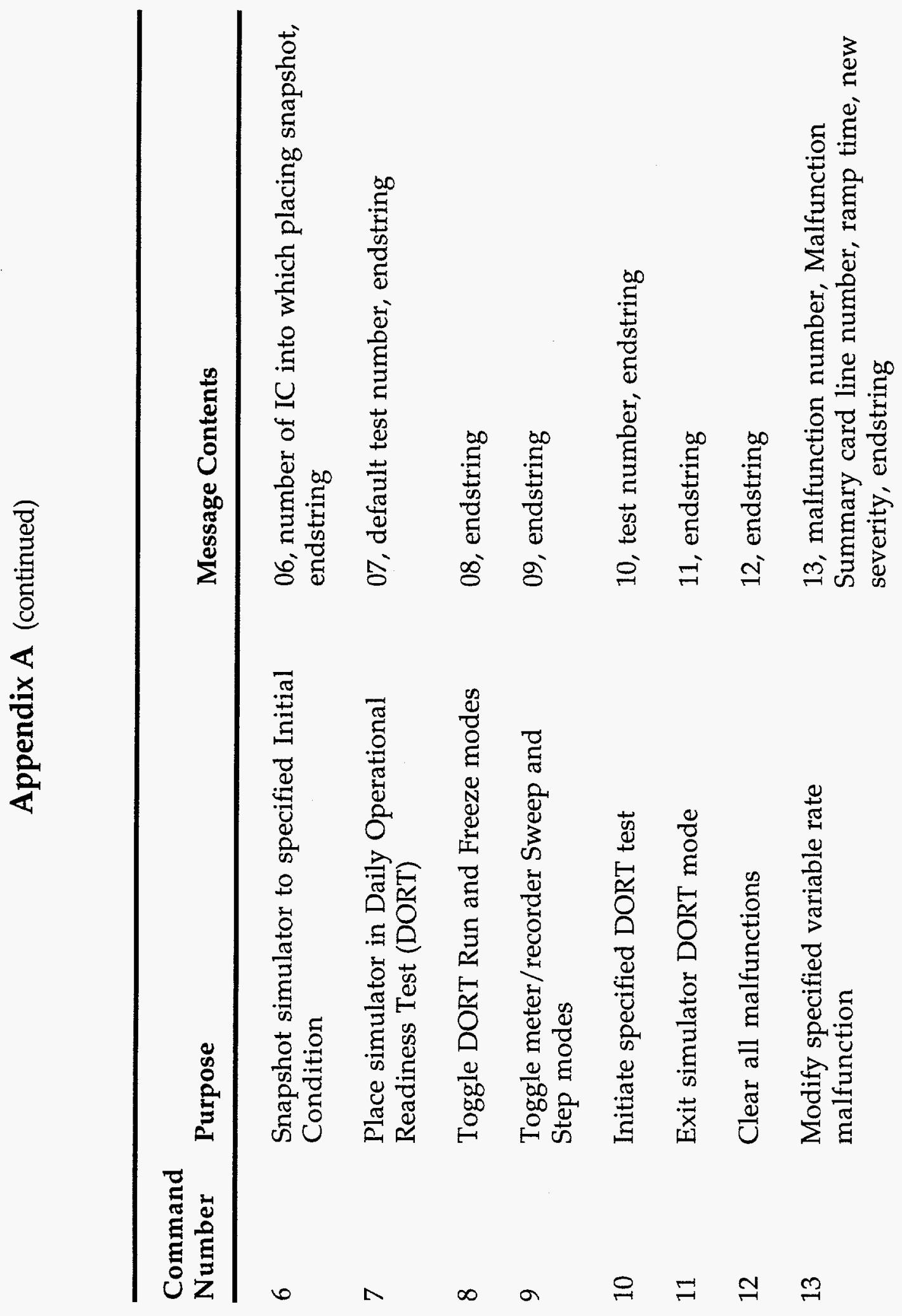




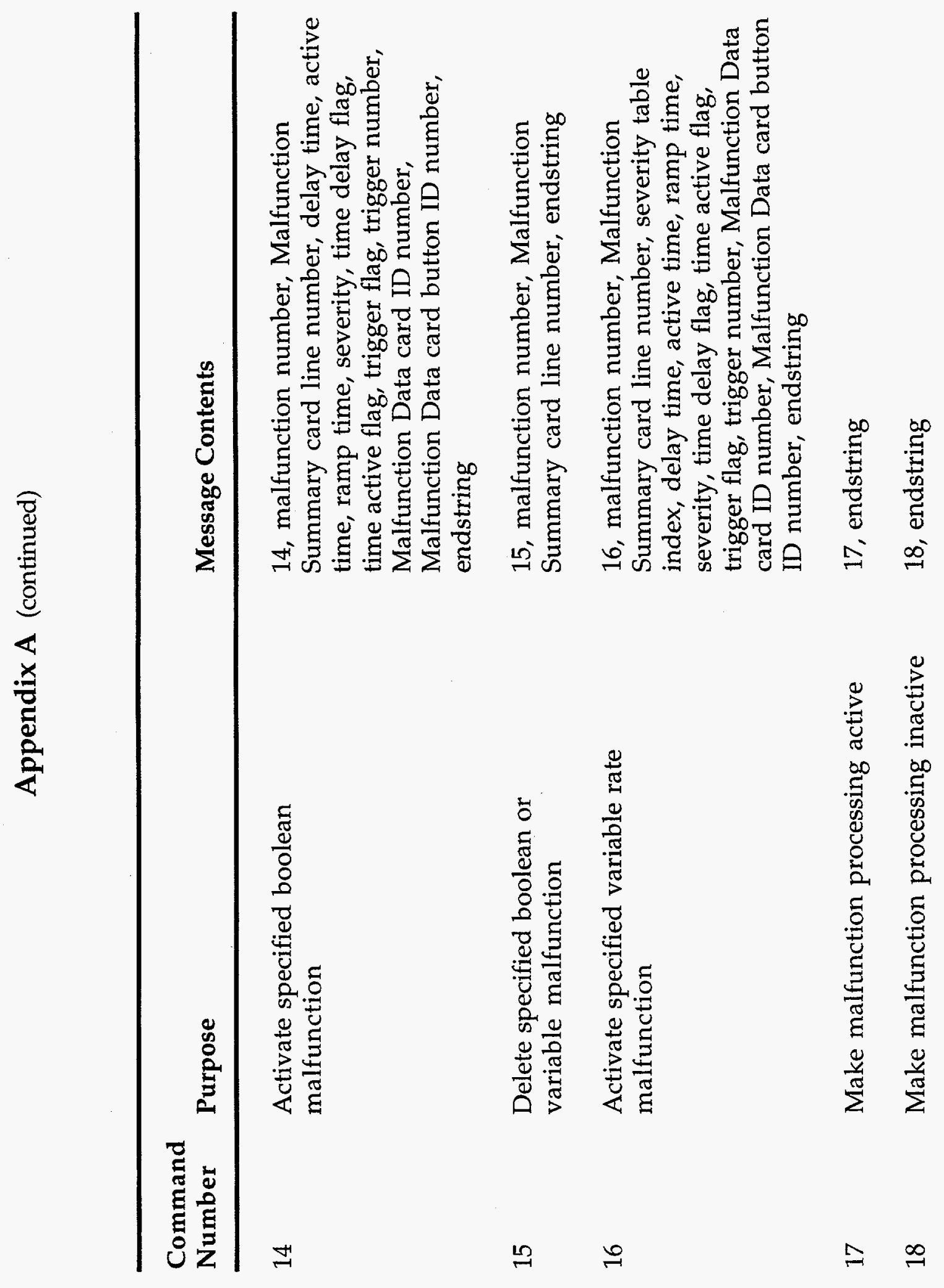


Appendix A (continued)

\section{Command}

Number Purpose

Message Contents

32 Get current severity of specified variable malfunction

32, malfunction number, severity table index, endstring

19 Turn remote function monitoring off

19 , endstring

20 Get current status of a string of boolean remote functions

20 , number of remote functions on the Remote Data card, remote function number(s), endstring

21 Toggle specified boolean remote function

21 , remote function number, state $(0$ or 1$)$, endstring

22 Get current status of a string of variable remote functions

22, number of remote functions on the Remote Data card, remote function value array pointer(s), endstring

23 Change specified variable remote function

23 , remote function value array pointer, value, endstring

Get current status of an individual boolean remote function

24 , remote function number, endstring 
Appendix A (continued)

\begin{tabular}{|c|c|c|}
\hline $\begin{array}{l}\text { Command } \\
\text { Number }\end{array}$ & Purpose & Message Contents \\
\hline 26 & Silence annunciator horn & 26 , endstring \\
\hline 28 & $\begin{array}{l}\text { Issue annunciator acknowledge and } \\
\text { reset }\end{array}$ & 28 , endstring \\
\hline 29 & Disable annunciator horn & 29 , endstring \\
\hline 30 & Enable annunciator horn & 30 , endstring \\
\hline 31 & Get current IC specific information & 31 , endstring \\
\hline 34 & Check status of request I/O override & 34 , override number, endstring \\
\hline 35 & Activate an I/O override & $\begin{array}{l}\text { 35, Override Summary card line number, type } \\
\text { of override commanded, override value, } \\
\text { Override Data card ID number, Override Data } \\
\text { card button ID number, endstring }\end{array}$ \\
\hline 36 & Delete an I/O override & $\begin{array}{l}36, \text { Override Summary card line number, } \\
\text { endstring }\end{array}$ \\
\hline 37 & Cancel I/O override setup & 37 , endstring \\
\hline
\end{tabular}


Appendix A (continued)

\section{Command}

Number Purpose

\section{Message Contents}

$38 \quad$ Clear all I/O overrides

38 , endstring

42 Get simulator status

42, simulator status flag, endstring

Sequence simulator software startup

44 , endstring

Sequence simulator software shutdown

45 , endstring

$47 \quad$ Snap a backtrack IC

Preview a backtrack IC

47 , number of IC into which placing backtrack snapshot, endstring

41 , number of backtrack IC wish to preview, endstring

46 , endstring

49 , time duration of recording, length of the name of the binary file in which the recorded data will be stored, the name of that binary file, endstring

54 , endstring 
Appendix A (continued)

\section{Command}

Number

Purpose

Message Contents

48

Activate data conversion program

Get more data acquisition data

Obtain status of identified variables selected for monitoring

48, ID number for group of points (version) wish to convert, flag indicating whether converting all the points in the group or only selected points, the number of points to be converted, the ID numbers of the points to be converted, length of the name of the binary file to be converted, the name of the binary file to be converted, endstring

55 , line number, endstring

56 , endstring

53 , endstring

57, ID number of component for which retrieving data, endstring

60 , number of identified variables selected for monitoring, list of array pointers for selected variables, endstring 
Appendix A (continued)

\begin{tabular}{|c|c|c|}
\hline $\begin{array}{l}\text { Command } \\
\text { Number }\end{array}$ & Purpose & Message Contents \\
\hline 61 & $\begin{array}{l}\text { Add a datapool variable to monitored } \\
\text { list }\end{array}$ & $\begin{array}{l}\text { 61, length of the name of the datapool va } \\
\text { to be monitored, the name of that datap } \\
\text { variable, Parameter Display card line nu } \\
\text { endstring }\end{array}$ \\
\hline 62 & $\begin{array}{l}\text { Update the status of the monitored } \\
\text { datapool variables }\end{array}$ & 62 , endstring \\
\hline 63 & Delete a monitored datapool variable & $\begin{array}{l}\text { 63, Parameter Display card line number, } \\
\text { endstring }\end{array}$ \\
\hline 64 & Clear all monitored datapool variables & 64, endstring \\
\hline 98 & Retransmit last message & 98 , endstring \\
\hline
\end{tabular}




\section{Appendix B}

\section{MACPCM Commands}

Messages from Host Computer to Macintosh Instructor Station

\begin{tabular}{|c|c|c|}
\hline $\begin{array}{l}\text { Command } \\
\text { Number }\end{array}$ & Message Contents & Format \\
\hline 1 & None & \\
\hline 2 & None & \\
\hline 4 & None & \\
\hline 50 & Endstring & 'PCM 50 ', R1 \\
\hline 51 & $\begin{array}{l}\text { Malfunction Summary card line } \\
\text { number for which getting data, } \\
\text { Malfunction Data card ID number, } \\
\text { Malfunction Data card button ID number, } \\
\text { malfunction type, malfunction number, } \\
\text { endstring }\end{array}$ & 'PCM 51 ‘,I2.2,1X,I3.3,1X,I3.3,1X,I1,1X,I4.4,1X,R1 \\
\hline 总 & $\begin{array}{l}\text { Override Summary card line number } \\
\text { for which getting data, Override Data } \\
\text { card ID number, Override Data card } \\
\text { button ID number, endstring }\end{array}$ & 'PCM $52^{\prime}, \mathrm{I} 2.2,1 \mathrm{X}, \mathrm{I} 3.3,1 \mathrm{X}, \mathrm{I} 3.3,1 \mathrm{X}, \mathrm{R} 1$ \\
\hline
\end{tabular}




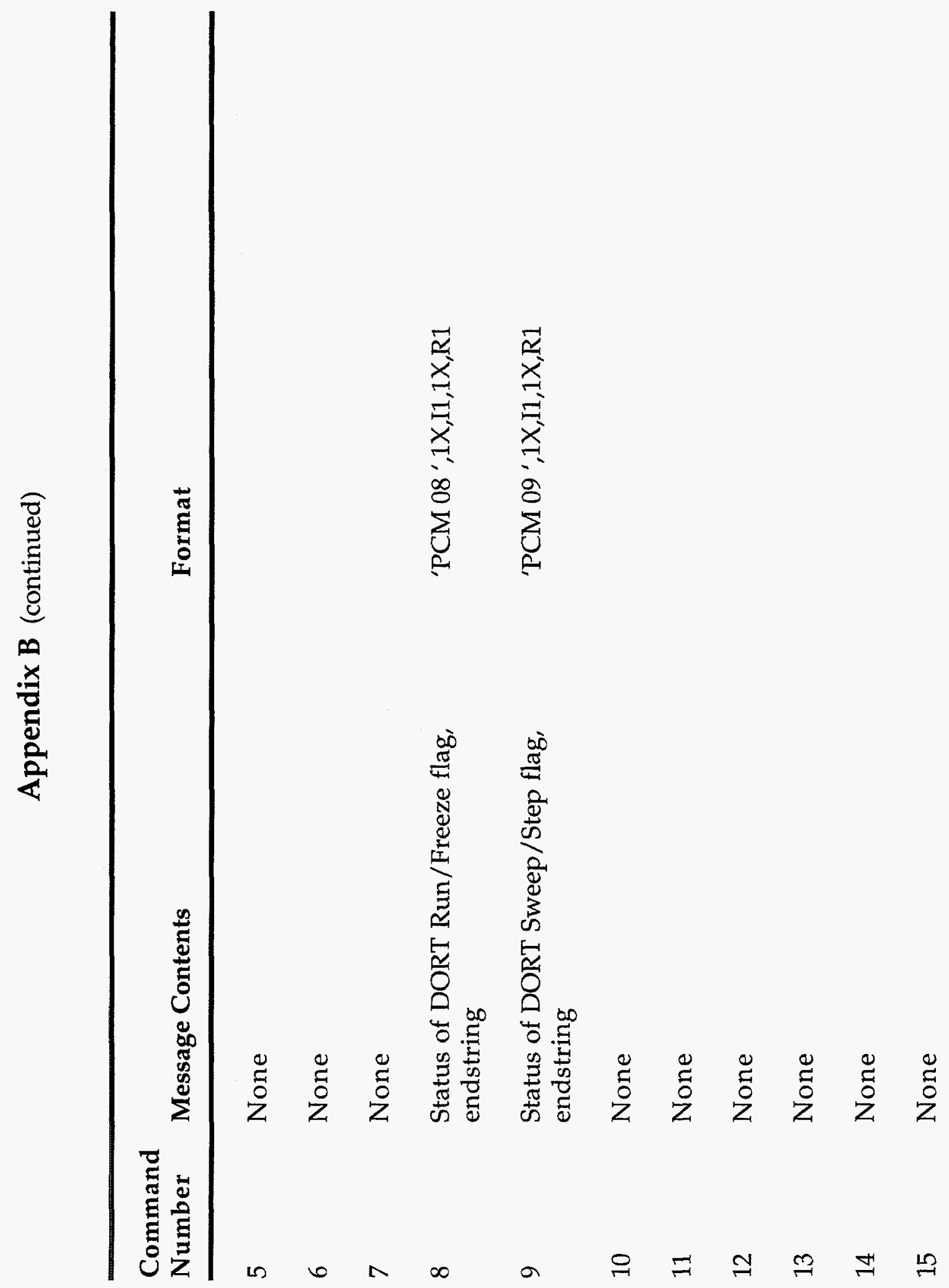




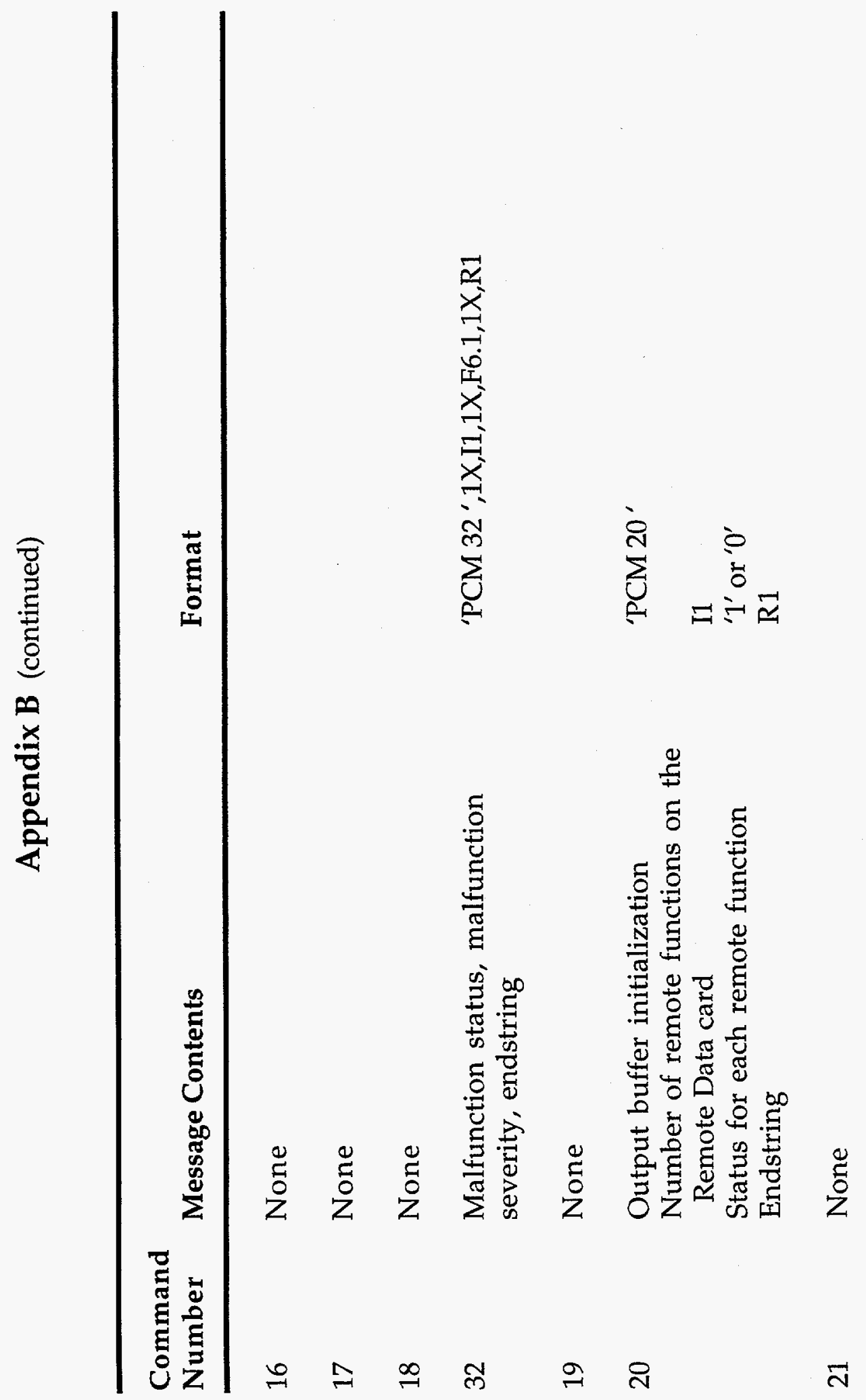


Appendix B (continued)

Command

Number

Message Contents

Format

22

Output buffer initialization

'PCM 22'

Number of remote functions on the

Remote Data card

I1

Value for each remote function

Endstring

F8.2

R1

里

23

None

24

Remote function status, endstring

'PCM 24 ',1X,I1,1X,R1

$26 \quad$ None

$28 \quad$ None

29 None

$30 \quad$ None

31 Parameter 1 value, Parameter 2

value, ..., endstring

'PCM 31 ',1X,F5.1,1X,F5.1,1X...,R1 


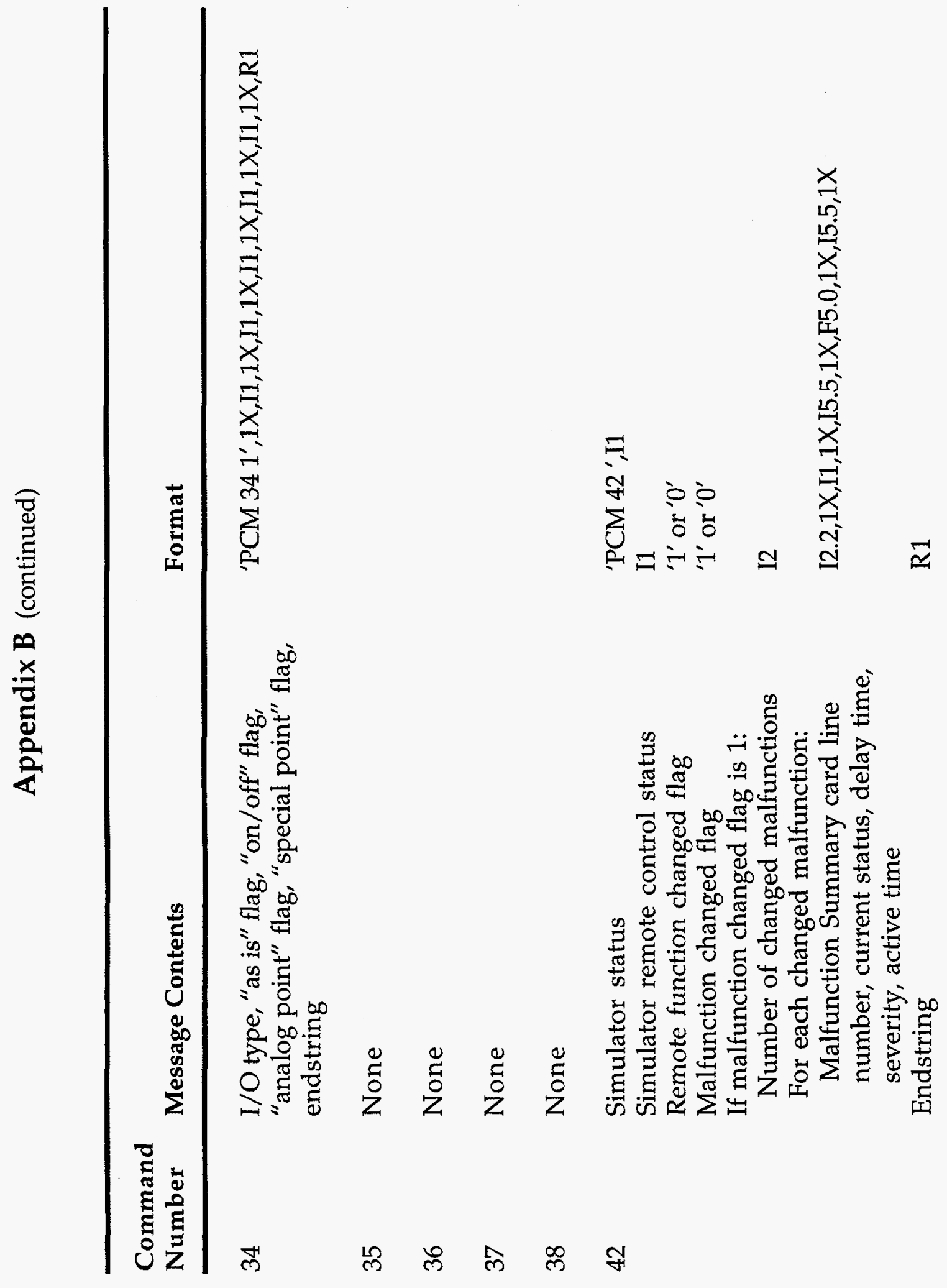


Command

Number

Message Contents

Format

$44 \quad$ Endstring

'PCM $44^{\prime}, \mathrm{R} 1$

$45 \quad$ None

$47 \quad$ None

必

41

None

46

None

49

Endstring

'PCM $49^{\prime}, \mathrm{R} 1$

54 If recording program still active:

Elapsed time, endstring

Otherwise:

Endstring

'PCM 54 1',1X,I5,1X,R1

'PCM 54 0',1X,R1

48

Error code, endstring

'PCM $48^{\prime}, 1 \mathrm{X}, \mathrm{I} 1,1 \mathrm{X}, \mathrm{R} 1$

55 None

$56 \quad$ None 


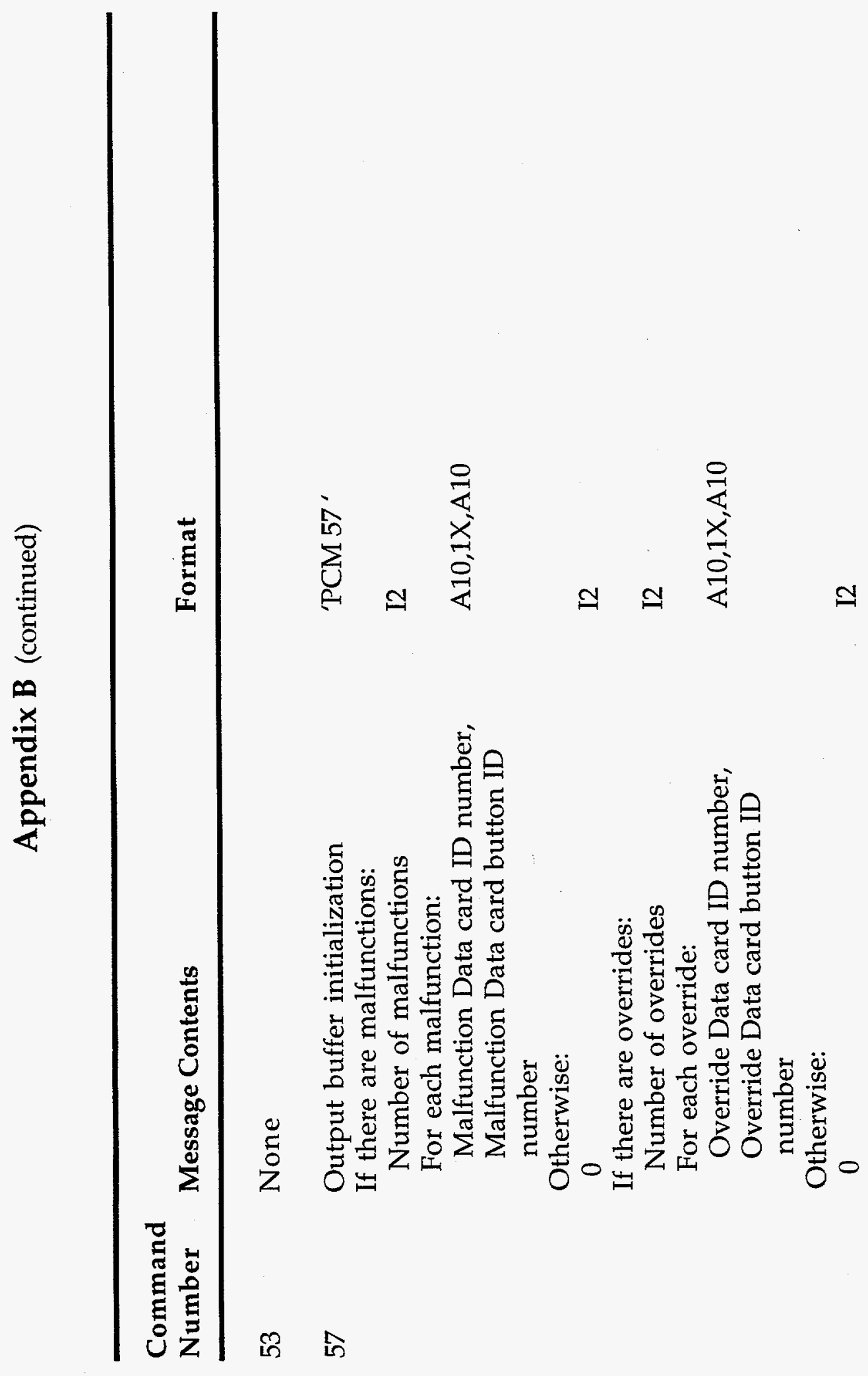




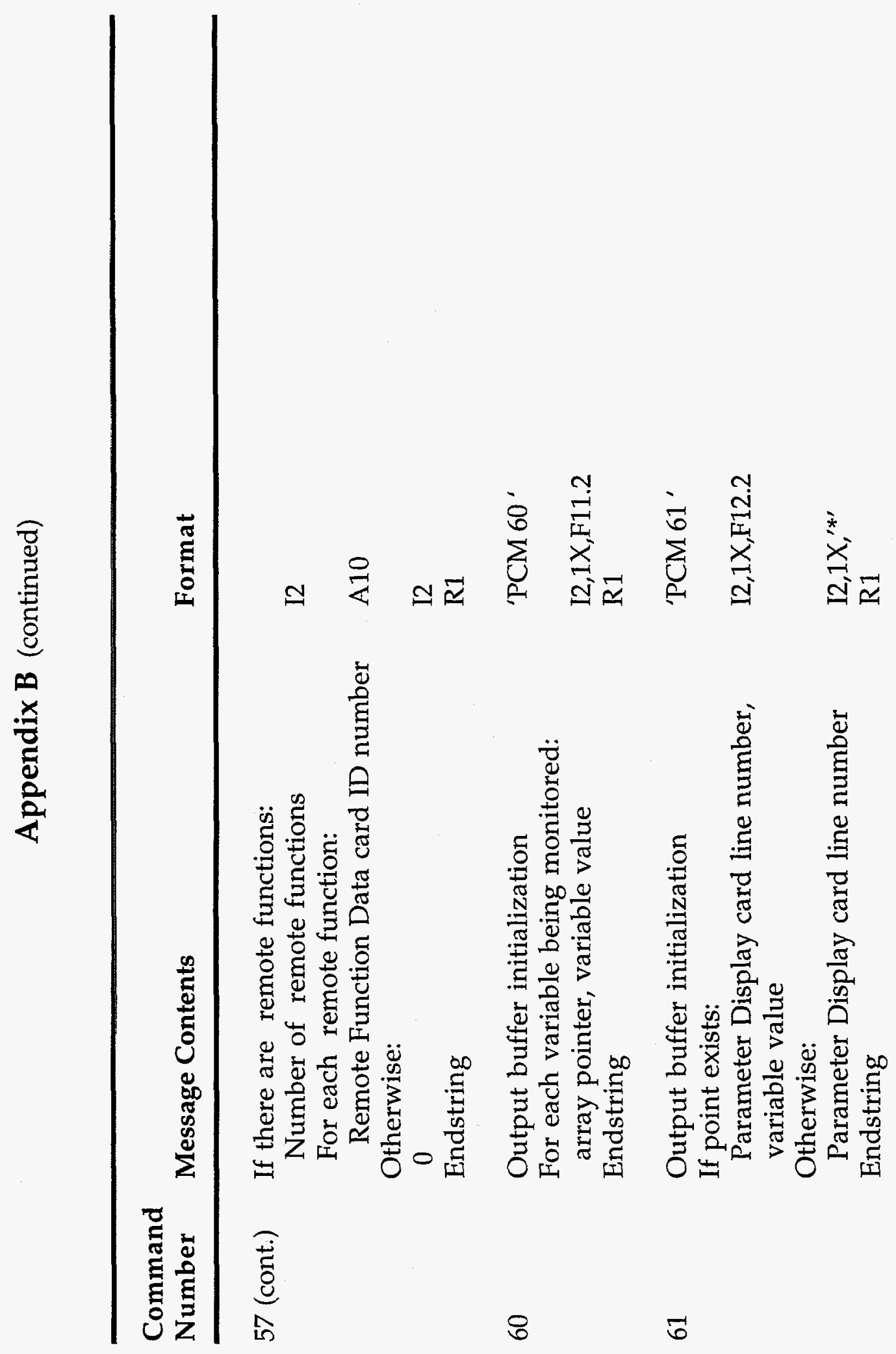




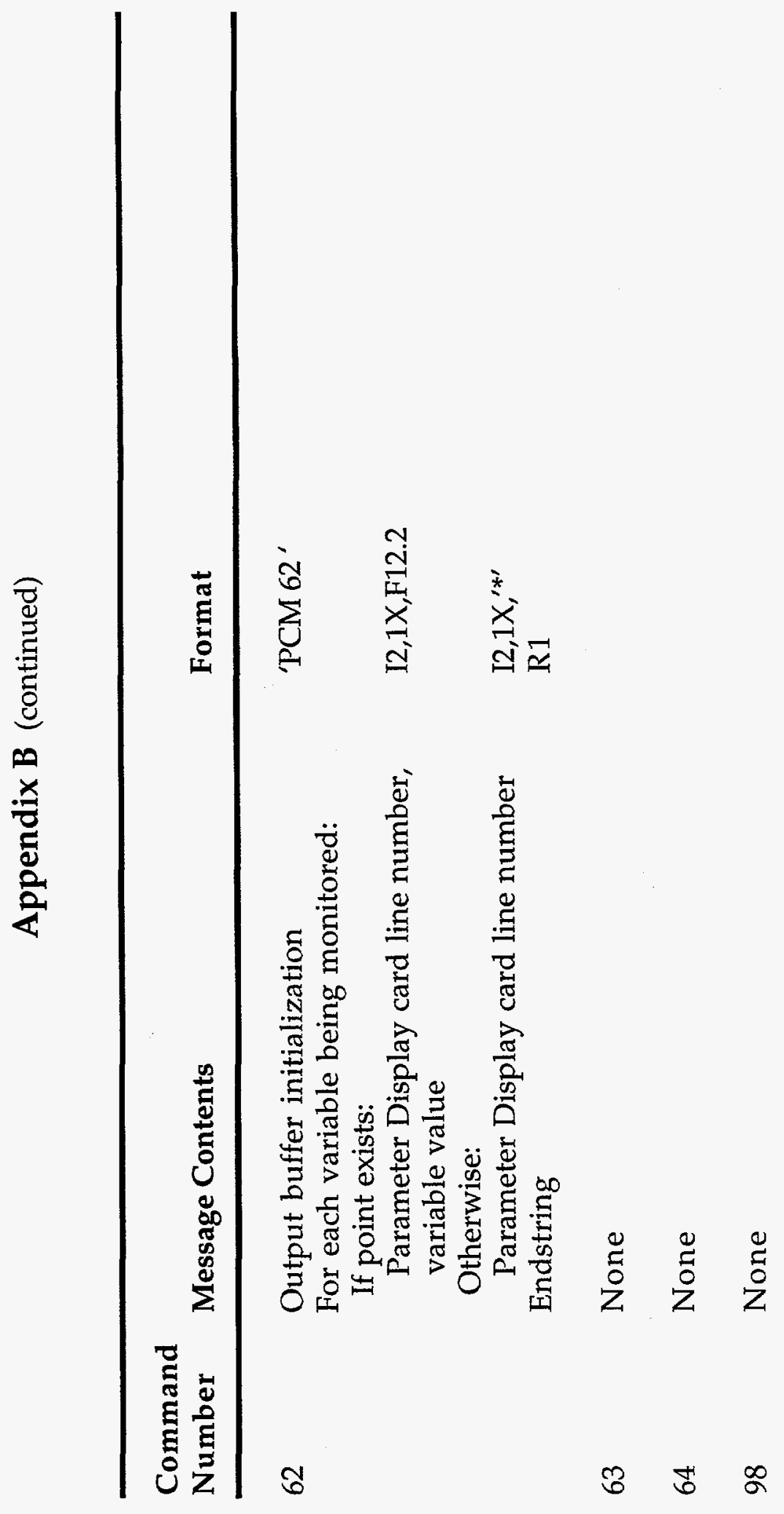


(See instructions on the reverse)

NRC's Object-Oriented Simulator Instructor Station

\begin{tabular}{|c|c|}
\hline 3. & \multicolumn{2}{c|}{ DATE REPORT PUBLISHED } \\
\hline MONTH & YEAA \\
June & 1995 \\
\hline
\end{tabular}

4. FIN OR GRANT NUMBER

Janice I. Griffin

Technical Report

James $P$. Griffin

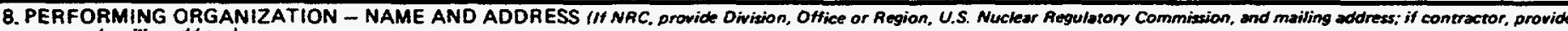
nome ond moiting address.

Technical Training Division

Office for Analysis and Evaluation of Operational Data

U.S. Nuclear Regulatory Commission

Washington, DC 20555-0001

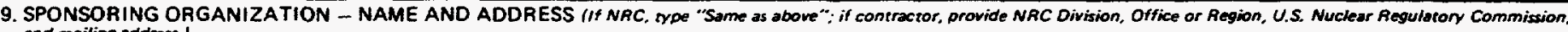
ind mailing addrest

Same as above

10. SUPPLEMENTARY NOTES

11. ABSTRACT 200 words or lossi

As part of a comprehensive simulator upgrade program, the simulator computer systems associated with the Nuclear Regulatory Commission's (NRC) nuclear power plant simulators were replaced. Because the original instructor stations for two of the simulators were dependent on the original computer equipment, it was necessary to develop and implement new instructor stations. This report describes the Macintosh-based Instructor Stations developed by NRC engineers for the General Electric (GE) and Babcock and Wilcox (B\&W) simulators.

\begin{tabular}{l} 
13. AVAILAGILITY STATEMENT \\
Unlimited \\
14. SECURITY CLASSIFICATION \\
\hline (This PAge) \\
Unclassified \\
(This RePOrt) \\
Unclas ified \\
15. NUMBER OF PAGES \\
16. PRICE
\end{tabular}

